NBSIR 75.823

\title{
HELIUM RESEARCH IN SUPPORT OF SUPERCONDUCTING POWER TRANSMISSION
}

V.D. Arp, D.E. Daney, N.V.Frederick,

M.C. Jones, P.R. Ludtke, W.R. Parrish and R.L. Powell

Cryogenics Division

Institute for Basic Standards

National Bureau of Standards

Boulder, Colorado 80302

October 1975

Prepared for :

Energy Research and Development Administration

Electrical Energy Systems Division

Washington, D.C. 20545 

NBSIR $75-823$

\section{HELIUM RESEARCH IN SUPPORT OF SUPERCONDUCTING POWER TRANSMISSION}

V.D. Arp, D.E. Daney, N.V.Frederick,

M.C. Jones, P.R. Ludtke, W.R. Parrish and R.L. Powell

Cryogenics Division

Institute for Basic Standards

National Bureau of Standards

Boulder, Colorado 80302

October 1975

Prepared for:

Energy Research and Development Administration

Electrical Energy Systems Division

Washington, D.C. 20545

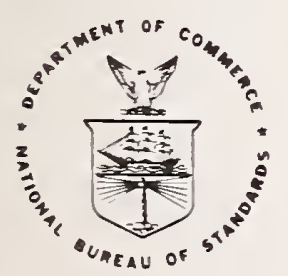

U.S. DEPARTMENT OF COMMERCE, Rogers C. B. Morton, Secretary John K. Tabor, Under Secretary

Dr. Betsy Ancker-Johnson, Assistant Secretary for Science and Technology 

CONTENTS

1. INTRODUCTION . . . . . . . . . . . . . . . . . . . . . . . . . . . . 1

2. THEORETICAL STUdiES OF COOLING SYSTEM DYNAMICS . . . . . . . . . . . . . . . . 1

2.1 Acoustic Oscillations . . . . . . . . . . . . . . . . . . . . . . 1

2.1.1 Acoustic Wave Propagation and Reflection in a Flow Channel . . . 3

2.1.2 Heat Driven Oscillations . . . . . . . . . . . . . . . . . 3

2.2 Negative Differential To Flow Resistance . . . . . . . . . . . . . . . 5

2.3 References . . . . . . . . . . . . . . . . . . . . . . 5

3. EXPERIMENTAL STUdIES OF COOLING SYSTEM DYNAMICS . . . . . . . . . . . . . . . . 6

3.1 Introduction . . . . . . . . . . . . . . . . . . . . . . . . 6

3.2 Pothead Modeling . . . . . . . . . . . . . . . . . . . . . . 6

3.3 Expeimental . . . . . . . . . . . . . . . . . . . . . . . 8

3.4 Progress . . . . . . . . . . . . . . . . . . . . . . . . . . . . 8

3.5 Nomencalture . . . . . . . . . . . . . . . . . . . . . . . 14

3.6 References . . . . . . . . . . . . . . . . . . . . . . . . . 14

4. HELIUM PRESSURE MEASUREMENTS IN SUPERCONDUCTING POWERLINES . . . . . . . . . . 15

4.1 Present Technology . . . . . . . . . . . . . . . . . . . . . . 15

4.2 Possibility of Thermodynamic Pressure Measurement . . . . . . . . . . . . 16

4.3 Microwave Pressure Transducer . . . . . . . . . . . . . . . . . . . 16

4.3.1 Schematic Pressure-Measurement System . . . . . . . . . . . . . 18

4.3.2 Cavity Tests . . . . . . . . . . . . . . . . . . . 18

4.3.3 Future Work. . . . . . . . . . . . . . . . . . . . . 21

4.4 References... . . . . . . . . . . . . . . . . . . . . . . . 24

5. TEMPERATURE MEASUREMENT . . . . . . . . . . . . . . . . . . . . . . . . . 25

5.1 Introduction . . . . . . . . . . . . . . . . . . . . . . . . 25

5.2 Types of Thermometers . . . . . . . . . . . . . . . . . . . . . 26

5.2.1 Active Electrical-Thermocouples . . . . . . . . . . . . . . . 26

5.2.2 Passive Resistive Electrical . . . . . . . . . . . . . . . . . 26

5.2.3 Passive Capacitive Electrical . . . . . . . . . . . . . . . . 28

5.2.4 Magnetic . . . . . . . . . . . . . . . . . . . . . . 28

5.2 .5 Fluid . . . . . . . . . . . . . . . . . . . . . 28

5.3 Effects of Electromagnetic Fields . . . . . . . . . . . . . . . . . . 29

5.4 Recommended Program . . . . . . . . . . . . . . . . . . . . . . . 33

5.4.1 Thermometer Adaptations . . . . . . . . . . . . . . . . . . . . 33

5.4.2 Signal Processing . . . . . . . . . . . . . . . . . . . . . 36

5.5 Conclusion and Future Plans . . . . . . . . . . . . . . . . . . . . . . 38

5.6 References. . . . . . . . . . . . . . . . . . . . . . . 39

6. IMPURITY PROBLEMS IN SUPERCONDUCTING TRANSMISSION LINES . . . . . . . . . . . . 40

6.1 Introduction . . . . . . . . . . . . . . . . . . . . . . . . . . . . 40

6.2 Accumulation of Impurities . . . . . . . . . . . . . . . . . . . . . . . . 40

6.3 Effect of Dispersed Impurities on Voltage Breakdown . . . . . . . . . . . 44

6.4 Methods to Minimize the Impurity Problem . . . . . . . . . . . . . . . . . 45

6.4.1 Purification of Helium Supply Gas . . . . . . . . . . . . . . . . . 45

6.4.2 Purification of Recirculating Helium . . . . . . . . . . . . . . . 46

6.4.3 System Configuration . . . . . . . . . . . . . . . . . . . 46

6.5 Conclusions and Recommendations . . . . . . . . . . . . . . . . . . . 47

6.6 References . . . . . . . . . . . . . . . . . . . . . . . 49

APPENDIX - NEGATIVE DIFFERENTIAL FLOW RESISTANCE IN SJ̈PERCRITICAL HELIUM • • • • • A-1 
2.1. Open loop gain in the region of acoustic resonance $v_{0} . . . . . . . . . . . .22$

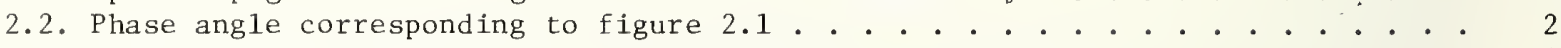

2.3. Phase relationships in heat driven acoustical oscillations . . . . . . . . . 4

2.4. System configuration for heat driven acoustical oscillations . . . . . . . 4

3.1. Pothead apparatus flow schematic . . . . . . . . . . . . . . . . 9

3.2. Pothead assembly drawing . . . . . . . . . . . . . . . . . . . . 10

3.3. Instrumented pothead without vacuum jacket . . . . . . . . . . . . . . . 11

3.4. Assembled pothead . . . . . . . . . . . . . . . . . . . . . . . . . 12

3.5. Pothead temperature profiles . . . . . . . . . . . . . . . . . 13

4.1. Velocity of sound in helium, and derivatives with respect to pressure and temperature, for selected pressures and temperatures . . . . . . . . . 17

4.2. UHF oscillator measurement system . . . . . . . . . . . . . . . . . . 19

4.3. $440 \mathrm{MHz}$ resonant cavity pressure sensor . . . . . . . . . . . . . . . . . . . 20

4.4. $369 \mathrm{MHz}$ resonant cavity pressure transducer . . . . . . . . . . . . . . . 22

4.5. $1200 \mathrm{MHz}$ resonant cavity pressure transducer . . . . . . . . . . . . . . . 23

5.1. Magnetoresistance of platinum resistors . . . . . . . . . . . . . . . 29

5.2. Magnetoresistance of germanium thermometers . . . . . . . . . . . . . . 31

5.3. Magnetoresistance of germanium thermometers . . . . . . . . . . . . . . . . . 31

5.4. Magnetoresistance of carbon resistor thermometers . . . . . . . . . . . . . . 32

5.5. Magnetoresistance of carbon resistor thermometers . . . . . . . . . . . . . 32

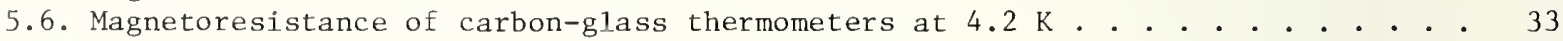

5.7. Thermometer encapsulation . . . . . . . . . . . . . . . . . . 35

5.8. Circuit for capacitance thermometers . . . . . . . . . . . . . . . . . 36

5.9 Methods of signal removal . . . . . . . . . . . . . . . . . . . . 37

6.1. The size of a sphere of impurity as a function of initial impurity concentration in the helium and helium capacity in the line . . . . . . . . . . 41

6.2. Effect of leak rate and operating time on the size of a sphere of solid air . . 42

6.3. Effect of oil concentration and operating time on the size of a sphere

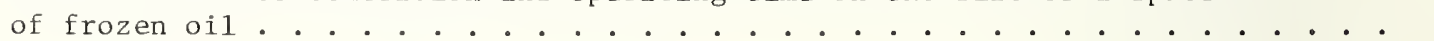

LIST OF TABLES

Table

Page

5.1 . . . . . . . . . . . . . . . . . . . 
V. D. Arp, D. E. Daney, N. V. Frederick, M. C. Jones,

P. R. Ludtke, W. R. Parrish and R. L. Powell

Cryogenics Division

Institute for Basic Standards

National Bureau of Standards

Boulder, Colorado 80302

\section{ABSTRACT}

This is the first annual report on a program of helium related research in support of superconducting power transmission. Program areas reported on are concerned with the dynamics of helium cooling systems -- theoretical aspects of oscillatory behavior and experiments -and helium related measurements. The latter include pressure measurement, temperature measurement and the problem of impurity levels in the helium refrigerant.

Key words: Cooling system; cryogenics; helium-cooled electrical leads; helium impurities; microwave cavities; negative differential flow resistance; pressure measurement; system dynamics; thermometers. 


\section{INTRODUCTION}

This is the first annual report of progress on research in support of superconducting power transmission carried out at the National Bureau of Standards, Cryogenics Division under ERDA, Division of Electric Energy Systems, sponsorship. Work was started under $\mathrm{AEC}$ and DoI funding received respectively in September and November 1974, so this first report covers between 8 and 10 months of work.

In this reporting period the major tasks undertaken were under categories described by Chapters 2, 3 and 4. These are respectively: i) Theoretical studies of the dynamics of the helium flow system which incorporates the cable and its terminations. We are concerned here with the potential for various types of oscillations, and the response to external perturbations. ii) Experimental studies in relation to system dynamics. We were directed first to study the stability of cable terminations (potheads) and then to consider the cable itself. iii) The development of methods of measurement of the helium cooling system parameters in the presence of electromagnetic fields of the power line.

In addition to these major tasks we were also to review and make recommendations on the problem of temperature measurement in the unique environment of a power transmission cable. The results of this review are given in Chapter 5. Finally, we were to review and recommend on the problem of impurities in the helium cooling system. This is presented in Chapter 6 .

\section{THEORETICAL STUDIES OF COOLING SYSTEM DYNAMICS}

\subsection{Acoustic Oscillations}

In a previous study [1] we had developed a rather general set of equations with which the dynamics of a helium cooling system could be investigated in the 1inear 1imit, i.e., small perturbations. These equations were used to investigate local stability about a steady state operating point of heated channels with typical power transmission line parameters. Computational methods were found which enabled accurate determinations to be made using the Nyquist plot at frequencies encompassing the density wave instability. However, when used at higher frequencies typical of acoustic resonance the numerical integration methods failed to produce convergent results in reasonable computation time. We therefore developed an approximate method whereby the integration could be performed analytically over a short length of channel where fluid property variation was negligible. Proceeding in short lengths along the entire channel, the channel transfer function could be rapidly computed and again the Nyquist plot of the system open loop transfer function could be made with any desired accuracy. The approximation basically neglected the fluid velocity relative to the acoustic velocity and became more accurate the higher the frequency. It was felt to be very adequate for typical transmission line parameters. We stress that this approximation did not affect the steady state situation about which stability was to be investigated. Thus the acoustic wave traveling along the channel still encountered the same variation of fluid states as before. We felt this to be a very realistic calculation.

A typical computation is illustrated in Figures 2.1 and 2.2 where frequency response is plotted. The open loop gain stays above 1.0 , but the phase angle remains below $\pi$ thus guaranteeing stability. Similar results computed for several different transmission lines were also invariably stable, and on this basis we had to conclude that helium cooled transmission lines would be stable against acoustic resonance. But this raised the question: why is it possible for acoustic oscillations to occur at all, as for instance in the experiments of Thurston and co-workers [2] using hydrogen, and Friedly [3] using nitrogen? We believe the key to this question lies in the interaction with the channel wal1.

In the next section we show that the computed stability at acoustic frequencies for helium-cooled transmission lines is in fact resonable. Following this we attempt to resolve the question raised. 


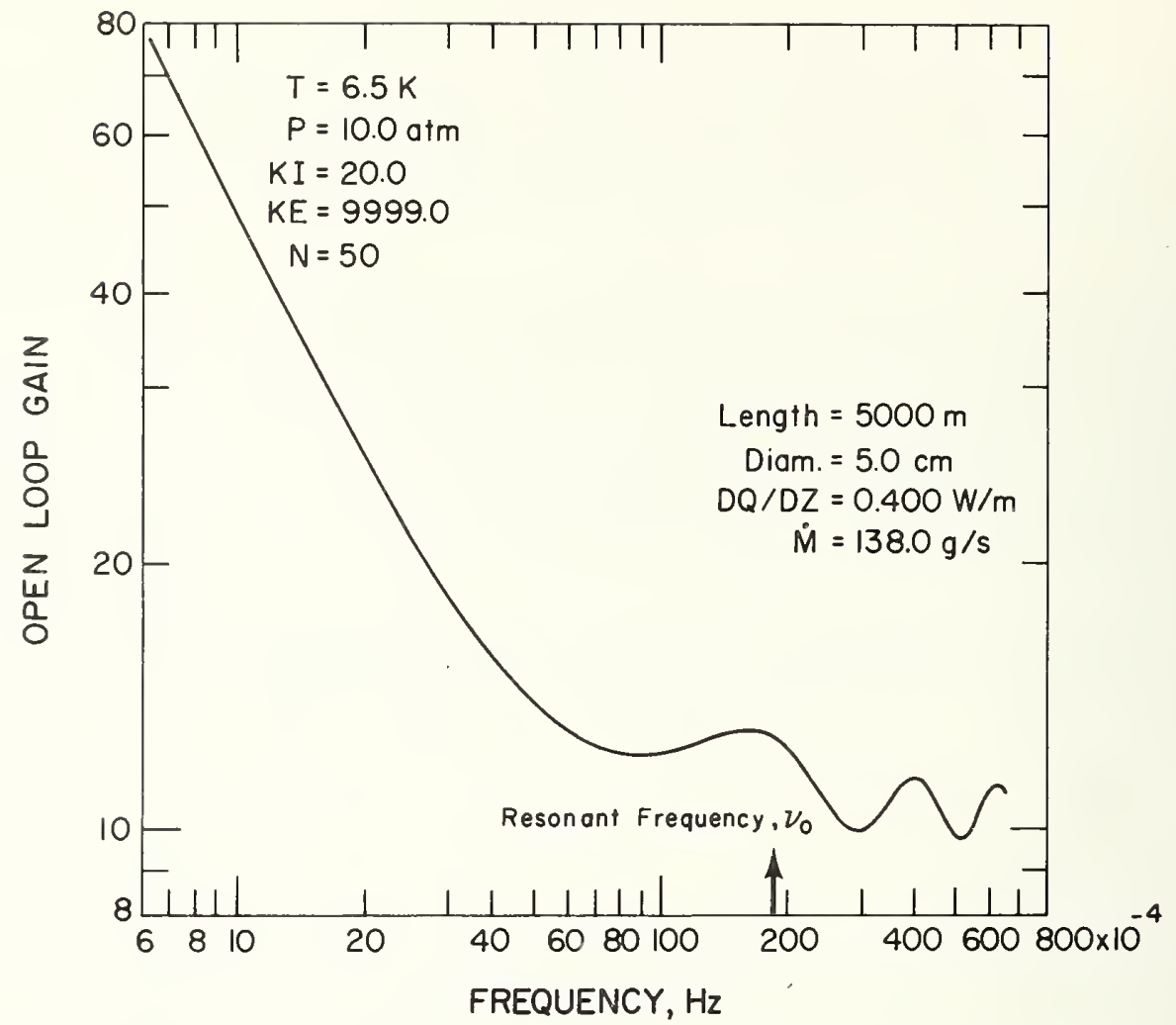

Figure 2.1 Open loop gain in the region of acoustic resonance $\nu_{0}$.

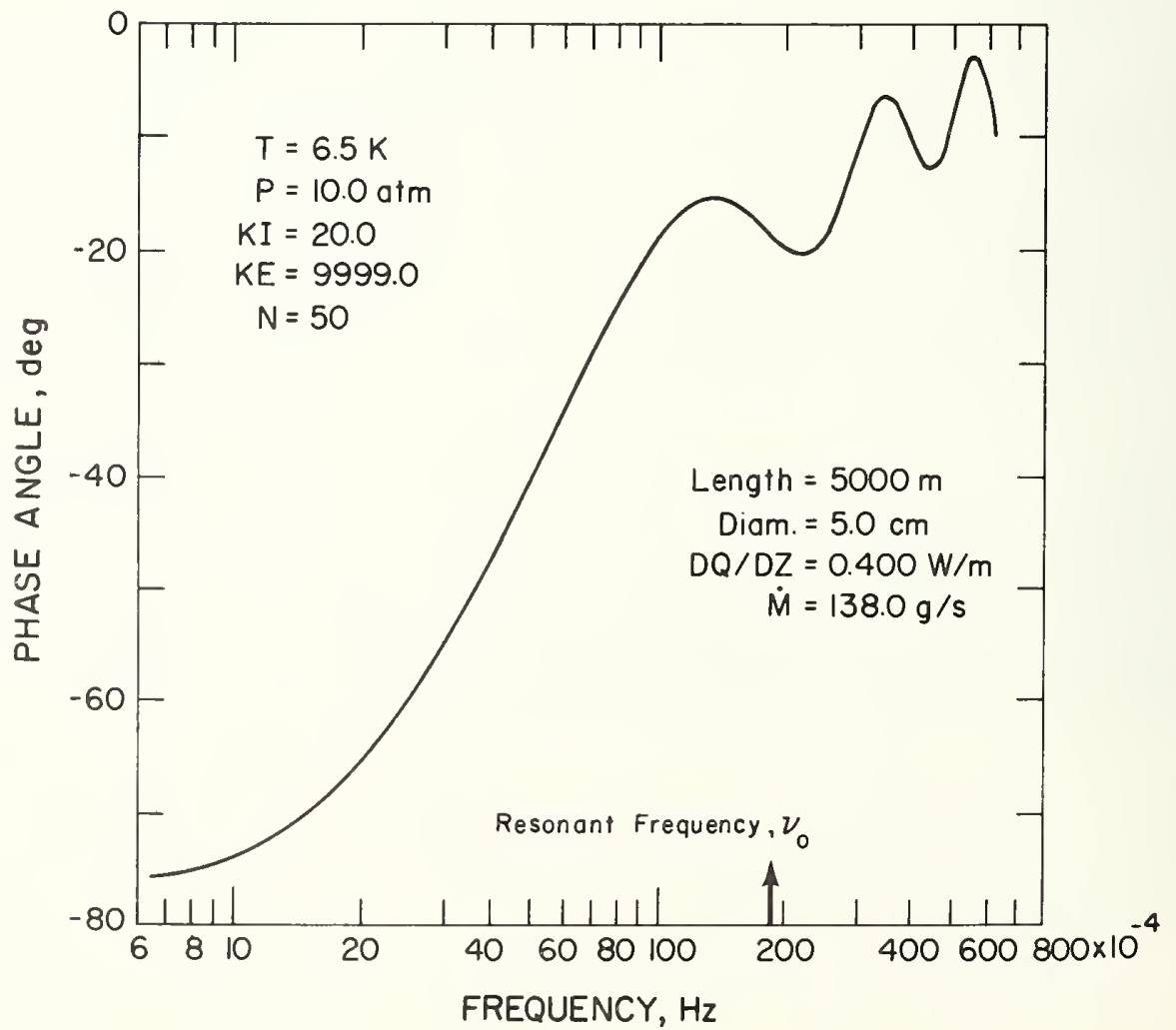

Figure 2.2 Phase angle corresponding to figure 2.1. 
2.1.1 Acoustic Wave Propagation and Reflection in a Flow Channel

Starting with the linearized time dependent continuity, energy and momentum equations developed in [1], if we assume that the mean fluid velocity $\bar{U}$ is small compared to the sonic velocity $C$, terms in $\partial \bar{U} / \partial x$ and $\bar{U} \partial / \partial x$. can be shown to be small compared to acoustic frequencies and thus for the dominant Fourier component w these may be dropped from the equations. The also drop terms resulting from interaction with the channel walls which is always permissible at helium temperatures. The resulting three equations can be used to elininate perturbations $\mathrm{H}$ in enthalpy and $\mathrm{U}$ in velocity to give the following wave equation for the pressure perturbation $\mathrm{p}$.

$$
\frac{\partial^{2} p}{\partial x^{2}}-b \frac{\partial p}{\partial x}-\frac{F \bar{U}}{C_{0}^{2}} \frac{\partial p}{\partial t}-\frac{1}{c_{0}^{2}} \frac{\partial^{2} p}{\partial t^{2}}=0
$$

where $b=\bar{c}(\partial v / \partial p)_{H} d / d x(\bar{p})$, and $C_{0}$ is the thermodynamic speed of sound: $C_{0}^{2}=(\partial p / \partial p)$, $\bar{p}$ is the steady state pressure and $\bar{p}$ the steady state density. $F=E P / a$ where $f$ is the friction factor for steady turbulent flow in the channel, $P$ is the perimeter and a the cross sectional area of this chanel. Now friction can certainly not contribute to amplification of an acoustic wave leading to an instability so we may set $F$ to zero. Then, if we find no mechanism for amplification and instability a finite $F$ will not change things.

Fourier analysis of the above equation with $p=p_{0} \exp -(k x-\omega t)$ leads to

$$
k=\frac{i b}{2} \pm \sqrt{\left(\frac{w}{c_{0}}\right)^{2}-\left(\frac{b}{2}\right)^{2}}
$$

where the + sign indicates a wave traveling in the tx direction and the - sign the - $\mathrm{x}$ direction. For all cases of interest at frequencies near the fundamental acoustical frequency, $\omega_{0}=2^{-} \mathrm{C} / 2 \mathrm{~L}$, the second term under the radical is much less than the $\mathrm{f} i \mathrm{~s}$ t. Iso, b Irom its definition above is a positive quantity. Thus the wave vector $k$ is that of a wave rraveling at a speed close to C, amplified in the positive $x$ direction and attenuated by like amount in the negative $x$ direction.

Consideration of the reflection of acoustic waves at flow restrictions or other cinannel discontinuities shows that the reflection coefficient is always less than unity. Then on a complete round trip within the channel a wave can only be damped. We see here no mechanist for net amplification and therefore no instability at these frequencies, in accord with our results from accurate numerical computation of stability criteria.

2.1.2 Heat Driven Oscillations

We consider that oscillations at acoustical frequencies when observed in higher temperature flow systems are of the heat driven kind. These were explained in the 19th century by Rayleign [-] when he noted that "if heat be given to the air at the moment of sreatest condensation (compression), or be taken from it at the moment of greatest rareiaction, the vibration is encouraged". The ingredients for such a heat driven oscillation are: i) flow of gas through the channel ii) heat input in a region where, in the standing wave pattern at resonance, all the gas particles are moving in the same direction iii) a channel wall with a high heat capacity, and iv) thermal diffusion time in the fluid much less than the period of oscillation. Items i) and ii) establish an axial temperature gradient in the Iluid, the level of which oscillates in the presence of a standing wave. I the heat input is in the $1 / 4$ wave section of the tube between a pressure node and antinode or a 1/2 wave section between two pressure antinodes then the phase relationship oi pressure, fluctuating component of velocity, residence time and temperature are as shetched in figure 2.3. Then, if through iii) the wall temperature is essentially constant the fluctuating component of heat flux seen by the gas is -hot where h is the heat transier coefficient, and is thus in phase with the pressure as required. In figure 2.4 possible configurations for heat driven oscillations are given. 


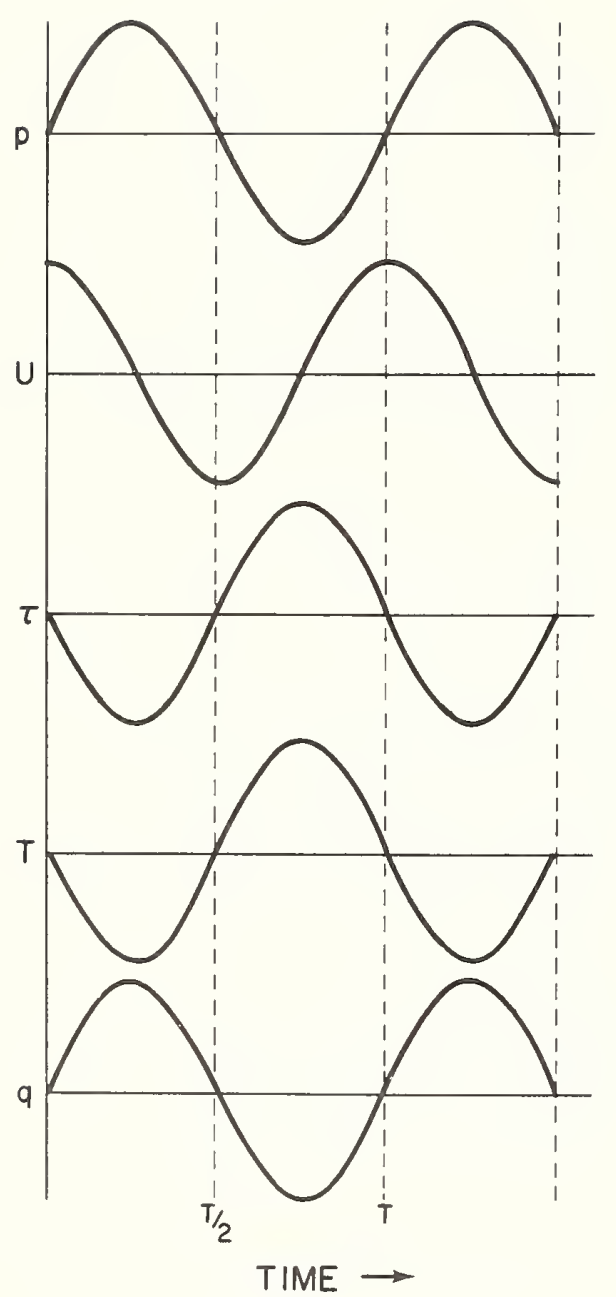

Figure 2.3 Phase relationships in heat driven acoustical oscillations. (a)

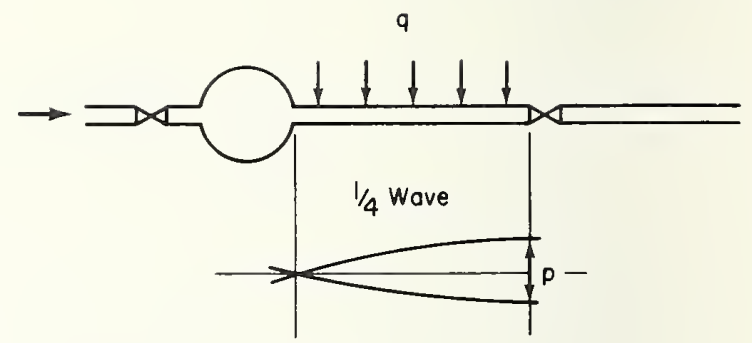

(b)

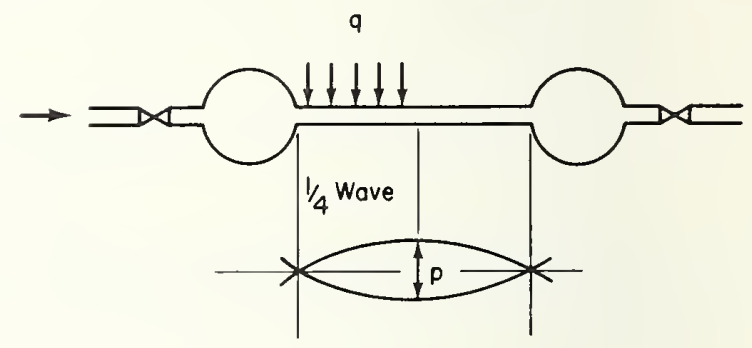

(c)

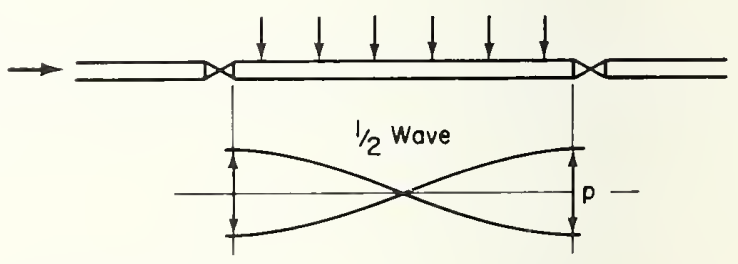

Figure $2.4 \quad$ System configuration for heat driven acoustical oscillations. 
We have not yet completed the generation of analytical criteria for the stability of this mode of oscillation, but we believe the circumstances of the experiments of Friedly and of Thurston support these ideas. Friedly observed acoustic resonance in the tube between a nitrogen gas reservoir and a flow restriction, case a. The standing wave pattern for this is a $1 / 4$ wave between the pressure node at the reservoir and the antinode at the flow restriction which gives unidirectional velocity fluctuaticns as required. Thurston had reservoirs at each end of his heated tube with flowing hydrogen, i.e., a $1 / 2$ wave section, case b. But it is significant that acoustic modes were mostly observed when the first $1 / 3$ alone was heated, and only rarely when the last $2 / 3$ were heated.

The essential difference between the helium-cooled transmission line and the above cases lies of course in the wall heat capacity. We do not believe that this type of oscillation is possible at liquid helium temperatures, and thus should not be important in the transmission line itself. However, helium cooled pot-heads will operate mostly at higher temperatures and have the right configuration, probably a. or c. Thether oscillations occur or not should depend on the thermal diffusion times from conductor to helium.

\subsection{Negative Differential To Flow Resistance}

Study begun some time ago on this problem under BNL support is now complete. A paper has been written on the subject by V. D. Arp and is included in this report as an appendix. The importance of this study is in two areas. In the first place the existence of negative differential flow resistance can lead to maldistribution in parallel channel flow. In the second place, based on high temperature experimental studies, [5] its existence in a flow channel appears to be a necessary condition for so-called pressure drop oscillations between a reservoir and the channel.

The results of our study may be briefly sumarized as follows:

Necessary and sufficient conditions have been derived for the existence of negative differential flow resistance. By approximate analysis and then exact numerical calculations it has been established that for helium the inlet thermodynamic states must be in a fairly restricted region below about $3.5 \mathrm{~K}$ and bounded by the two-phase coexistence line. For single-phase cooling systems it will only occur below about 3 atm pressure, with very high heat input, short channel lengths and in the absence of exit pressure drop restrictions. The phenomenon will not arise in helium-cooled superconducting transmission lines.

These conclusions are in accord with calculations performed earlier in the frequency domain by Nyquist analysis. For negative differential flow resistance the phase lag of the system should be - instead of zero as the frequency goes to zero. This circumstance never arose with parameters representative of superconducting power transmission.

\subsection{References}

1. Jones, Y. C. and Peterson, R. G., A study of flow stability in helium cooling systems, ASYE paper no. 74-WA/HT-24.

2. Thurston, R. S., Thermal-acoustic oscillations induced in forced convection heating of dense hydrogen, Ph.D. dissertation, University of New Yexico and Los Alamos Scientific Laboratory Report LA-3543 August 26, 1966.

3. Krishnan, V. S. and Friedly, J. C., Pressure oscillations in forced convection heating of gases, paper presented at the International Heat Transfer Conference, Tokyo, 1974.

t. Rayleigh, J. W. S., The theory of sound, Vo1. II, p. 226 (Dover Publications, X.Y., 1945).

5. Maulbetsch, J. S, and Griffith, P., Prediction of the onset of system induced instabilities in subcooled boiling, Symposium on Two-Phase Flow Dynamics Vol. EURATOM Report 4288e, Brussels, 1969. 


\section{EXPERIMENTAL STUDIES OF COOLING SYSTEM DYNAMICS}

\subsection{Introduction}

The purpose of the initial part of this study is to investigate flow stability in potheads (gas cooled current leads) for superconducting power transmission lines (SPTL). These potheads, which provide the link between the ambient conductor $(300 \mathrm{~K})$ and the superconductor ( 4 to $10 \mathrm{~K}$ ), may be subject to two types of system instability: thermal runaway instability and oscillatory instability.

Thermal runaway instability occurs when there is strong coupling between the total pothead flow resistance and the pothead temperature. Under these circumstances, a small current overload will warm the pothead, which increases the flow resistance and reduces the coolant flow. Further warming and flow reduction occur until the metallurgical limit of the conductor is reached and it melts. When an adequate pressure difference is avai1able, the simple solution to this type of system instability is to place a metering valve (having a flow impedance large relative to that of the pothead) at one end of the pothead. Temperature feedback control of the metering valve should further increase the stability of the system. Because most gas cooled current leads presently in operation are cooled by liquid helium boil-off gas, they operate with insufficient pressure for a metering valve system. Superconducting transmission lines, however, will operate at elevated pressure and are well suited to metering valve control of the pothead flow. Therefore thermal runaway instability should not be a serious problem for SPTL potheads.

Oscillatory instability, however, could be a serious problem. Helium systems are notorious for so-called thermo-accoustical oscillations in passages connecting ambient and helium temperatures [1-4], which is precisely the configuration of a pothead. Although such oscillations have not generally been a problem for boil-off gas cooled current leads, the danger of such an instability may be much higher for SPTL potheads operating at supercritical pressures. Potheads with inlet temperatures at or below the transposed critical temperature may be particularly susceptible to instability since the transposed critical line will be crossed somewhere in the pothead with consequent large volume change.

The detailed objective of this experimental investigation is to map the regions (pressure and temperature) of stability for a variety of pothead configurations and operating conditions (current and flow rate).

\subsection{Pothead Modeling}

Because full scale SPTL's are envisioned with operating currents of $10^{4}$ amps or more, economics dictate testing pothead models rather than full scale prototypes. To assure the validity of these model tests we ise scaling parameters which are derived from the differential equations of the pothead. First law analysis gives

$$
\frac{\partial}{\partial x}\left(k A \frac{\partial T}{\partial x}\right)-P h(T-\theta)+\frac{t^{*} T^{2}}{A}-\left(\frac{C A}{v_{S}}\right) \frac{\partial T}{\partial t}=0
$$

for the conductor, and

$$
\dot{\mathrm{m}} \frac{\mathrm{dH}}{\mathrm{dx}}=\mathrm{Ph}(\mathrm{T}-\theta)
$$

for the gas; or assuming

$$
\mathrm{dH}^{\mathrm{N}}=\mathrm{C}_{\mathrm{P}} \mathrm{d}^{\ominus}
$$

equation [3.2] becomes

$$
\frac{\mathrm{d} \theta}{\mathrm{dx}}=\frac{\mathrm{Ph}}{\dot{\mathrm{m} r}} \underset{\mathrm{p}}{\mathrm{p}}(\mathrm{T}-\theta)
$$


If we further assume
1) steady state,
2) I $-\theta=$ constant,
3) that the Wiedeman-Franz-Lorenz Law applies, viz.,

$$
L_{0}=\frac{k p}{T}
$$

4) $t^{*}=t$, i.e., the thickness of the conductor does not exceed the AC skin depth

5) constant conductor cross-sectional area,

equations (3.1) and (3.3) yield

$$
\frac{\partial}{\partial x}\left(k \frac{\partial T}{\partial x}\right)-\frac{\dot{m} C}{A} \frac{\partial T}{\partial x}+\frac{L_{o} I^{2}}{k A^{2}} T=0
$$

Or defining a dimensionless length $\mathrm{y}=\mathrm{x} / \mathrm{l}$, we obtain

$$
\frac{\partial}{\partial y}\left(k \frac{\partial T}{\partial y}\right)-\left(\frac{C \dot{m} \ell}{A}\right) \frac{\partial T}{\partial y}+\left(\frac{L I^{2} \ell^{2}}{k A^{2}}\right) T=0 .
$$

Identical dimensionless profiles require that the coefficients in equation (3.5) be constant, i.e.,

$$
\frac{\dot{m} C}{A} \hat{\imath}=\text { constant, and } \frac{L}{k} \frac{I^{2} \imath^{2}}{A^{2}}=\text { constant. }
$$

Assuming use of the same material and fluid in the model and prototype, these conditions become

$$
\frac{1}{A}=\text { constant }
$$

and

$$
\frac{\dot{m}}{\mathrm{~A}}=\text { constant }
$$

or

$$
\frac{\dot{m}}{I}=\text { constant. }
$$

If we nake the length of the model identical to that of the prototype, then the requirements for identical axial temperature profiles in the model and prototype are simply

and

$$
I / A=\text { constant }
$$

$$
\dot{m} / \mathrm{A}=\text { constant. }
$$


In addition to maintaining the same axial temperature distribution in the model pothead, we also wish to maintain dynamic similarity by maintaining the flow impedance dp/du constant [5] and to maintain hydrodynamic similarity by maintaining the Reynolds number constant. For configurations such as multiple strands of braided wire (e.g., Efferson leads [6]), both dynamic similarity and hydrodynamic similarity are maintained over a wide range of conductor areas, provided the conductor matrix (wire size and packing density) remain unchanged. Because exact thermal and dynamic modeling are not always possible, we plan to test pothead models with a range of flow impedances. Thus, the first pothead tested is a hollow tube, which represents the low impedance limit; higher impedance models will follow.

\subsection{Experimental}

Figure 3.1 is a schematic of the apparatus which provides helium to the model potheads at temperatures down to $4 \mathrm{~K}$ and pressures up to $20 \mathrm{~atm}$. High pressure helium gas passes through an ambient temperature purifier and is then cooled to $80 \mathrm{~K}$ and further purified in the liquid nitrogen cooled precooler. The helium then flows through a vacuum insulated line to the pothead dewar where it is further cooled as it passes through a counterflow heat exchanger. Final temperature control is achieved with the flow splitting valve which directs up to 100 percent of the flow through a coil immersed in the liquid helium refrigerant bath. Instead of entering the potheads directly, the helium passes through a 15 liter reservoir so as to provide a low dynamic flow impedance source.

Currents up to 2000 amps are supplied by a current transformer. Current measurement is accomplished with a current transformer in combination with a one ohm standard resistor and a peak voltage detector.

The apparatus is designed so that a variety of potheads may be tested. Conductor diameters in excess of $3 \mathrm{~cm}$ and lengths over $1 \mathrm{~m}$ can be accommodated. Figure 3.2 is an assembly drawing of the first pothead model to be tested. The conductor is a 4.76-mm I.D. x $0.25-\mathrm{mm}$ wall $\mathrm{x} 46-\mathrm{cm}$ long tube of phosphorized copper with a nominal current capacity of 100A. Germanium resistance thermometers measure the temperature at the base of the conductor and the temperature of the helium coolant. Ten gold $(0.07$ at. percent iron) vs KP thermocouples, which are referenced to the base of the pothead, measure the conductor temperature along its length. Current, helium, flow rate, pressure and pressure drop are also measured. Figure 3.3 shows the instrumented pothead before assembly of the vacuum jacket, and figure 3.4 shows the completely assembled and instrumented pothead ready for insertion into the apparatus. Because each pothead is assembled as a separate unit, they may be interchanged with a minimum of effort and down-time.

The second pothead constructed serves as the return current lead and consists of a $45.7 \mathrm{~cm}$ length of braided copper wire (192 strands, 34 gauge). The braids lie in the annular space between a $4.8 \mathrm{~mm}$ diameter stainless steel tube and a $3.2 \mathrm{~mm}$ diameter micarta* rod which forces the helium flow into the antulus. Self-regulated helium flow through this lead is accomplished with a flow control valve actuated by a nitrogen vapor pressure bulb.

A series of potheads will be tested, and although the design shown in figures 3.2 through 3.4 may never be used in practice, its importance is that it represents the low pressure drop limit. The second pothead to be instrumented and tested will be of the braided wire construction described above, and other potheads will be chosen to model specific potheads proposed by the various SPTL groups as their designs become available.

\subsection{Progress}

The apparatus, instrumentation, and data reduction program are complete, and an initial shake down run at helium temperatures has been completed. Only a few minor problems occurred, and it is anticipated that on the next experimental run (planned for the immediate future), mapping of the region of stability for the first pothead will begin. Data acquisition and reduction are accomplished by a mini-computer which provides on-line data reduction. The complete, current temperature profile of the pothead is always available to the operator, making the control of the experiment, and selection of the correct operating point quite easy. 


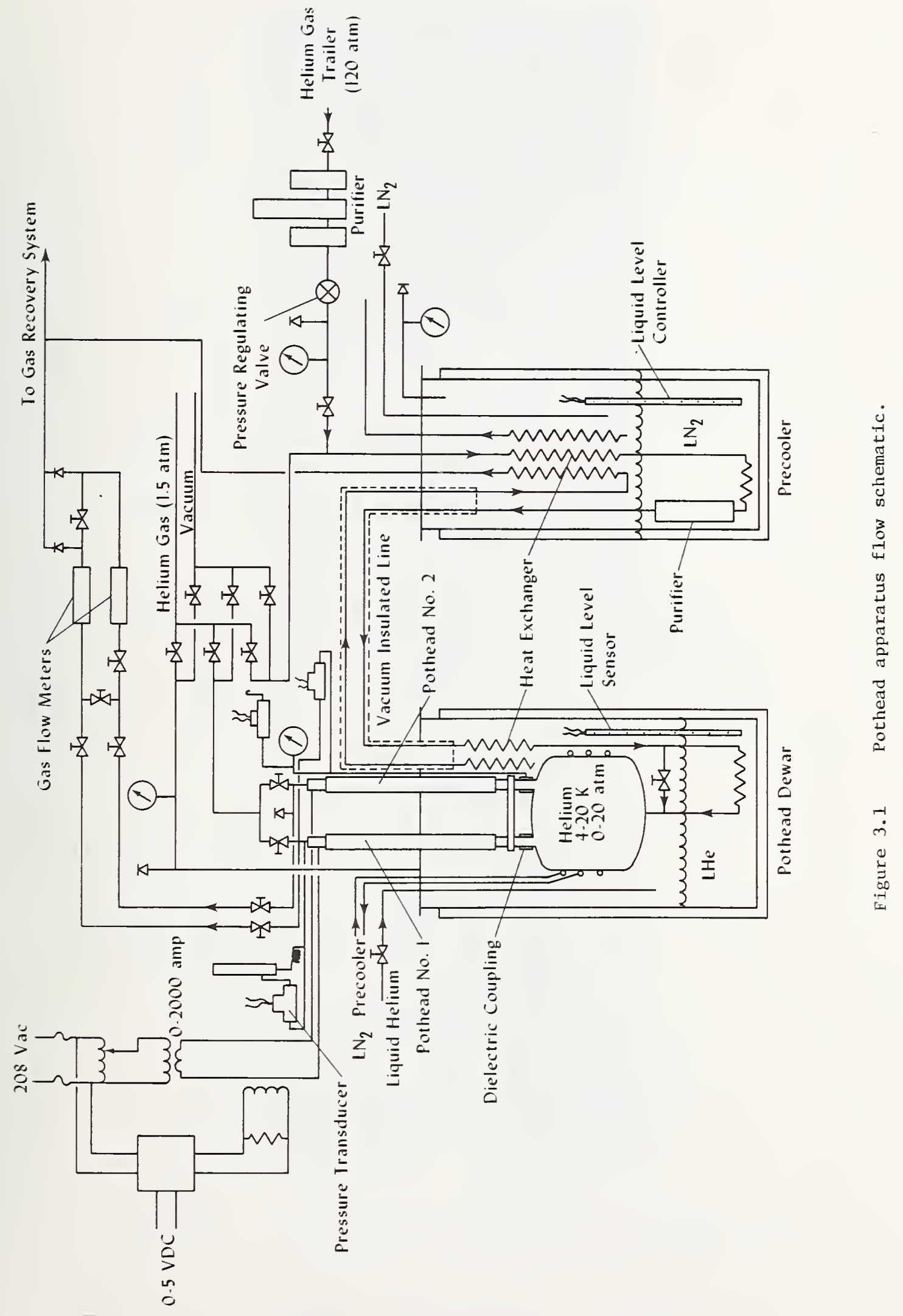




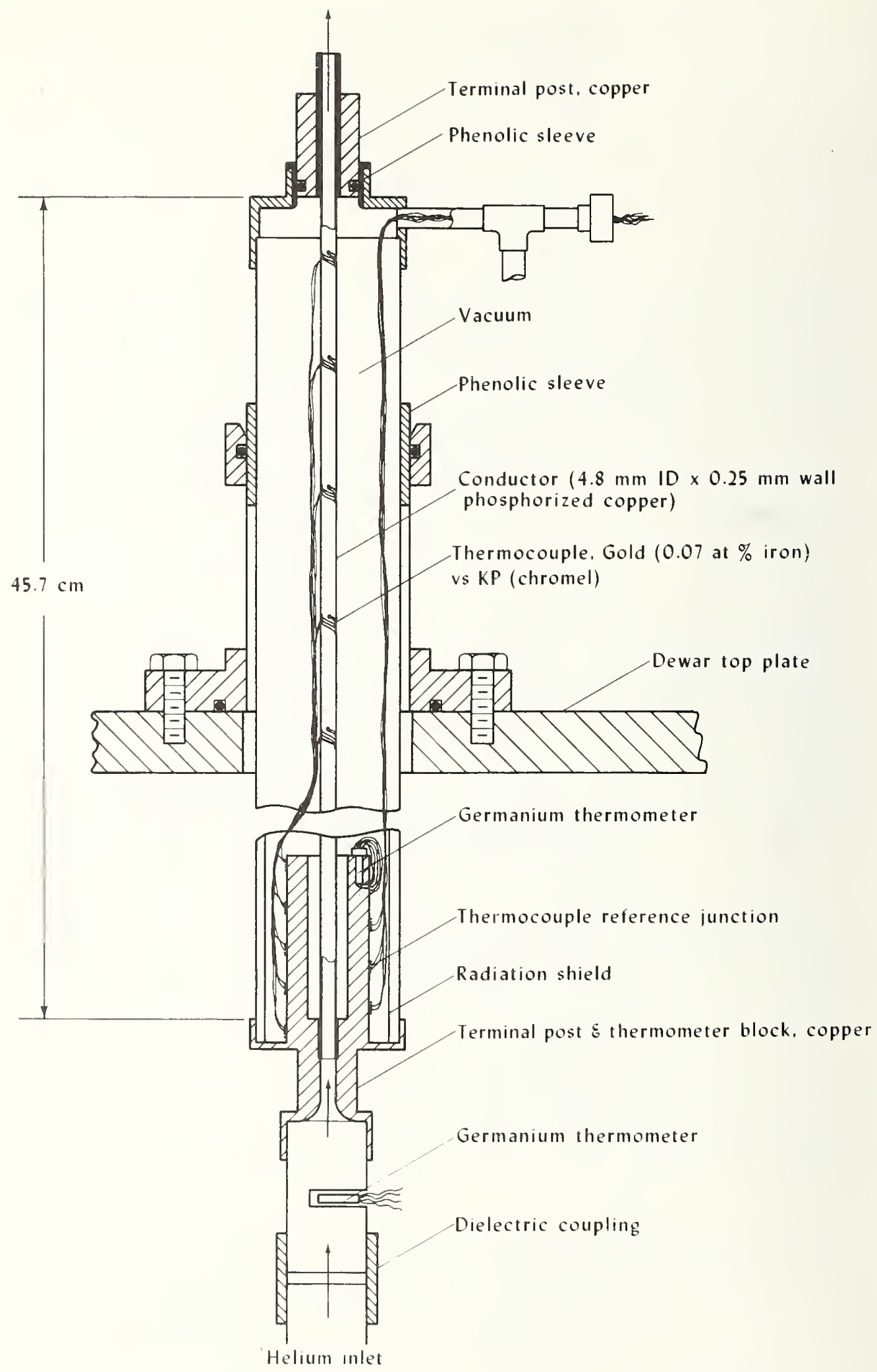

Figure 3.2 Pothead assembly drawing. 


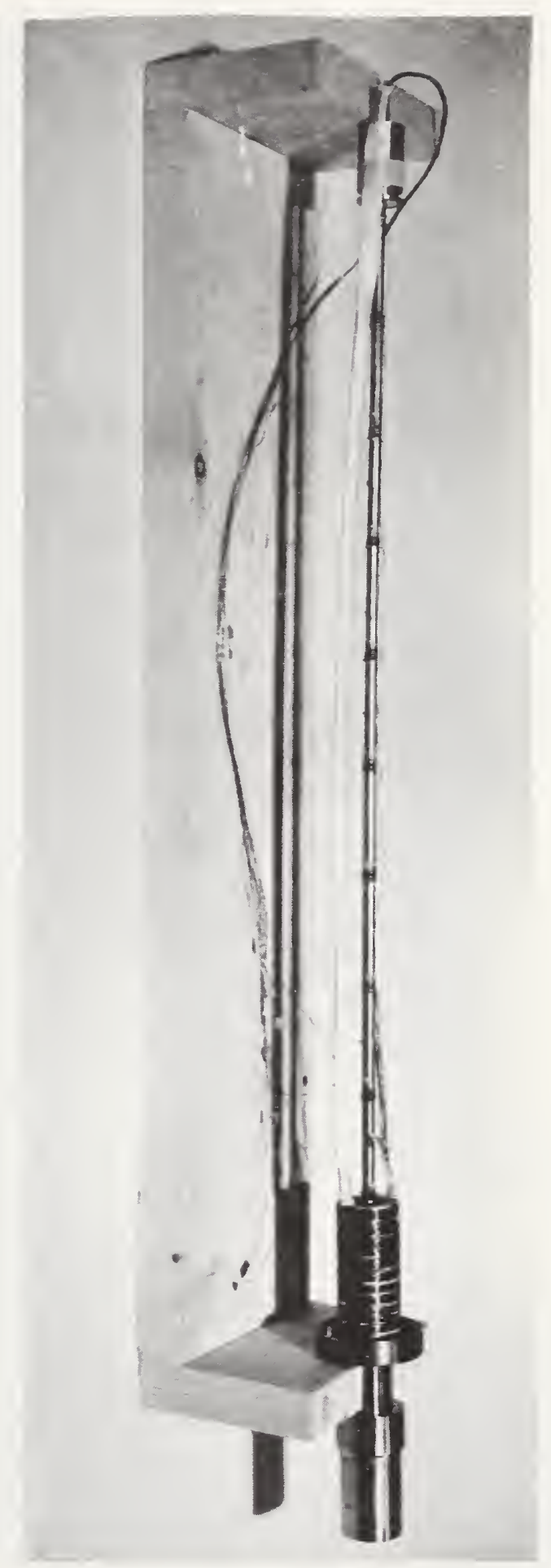

Figure 3.3 Instrumented pothead without vacuum jacket. 


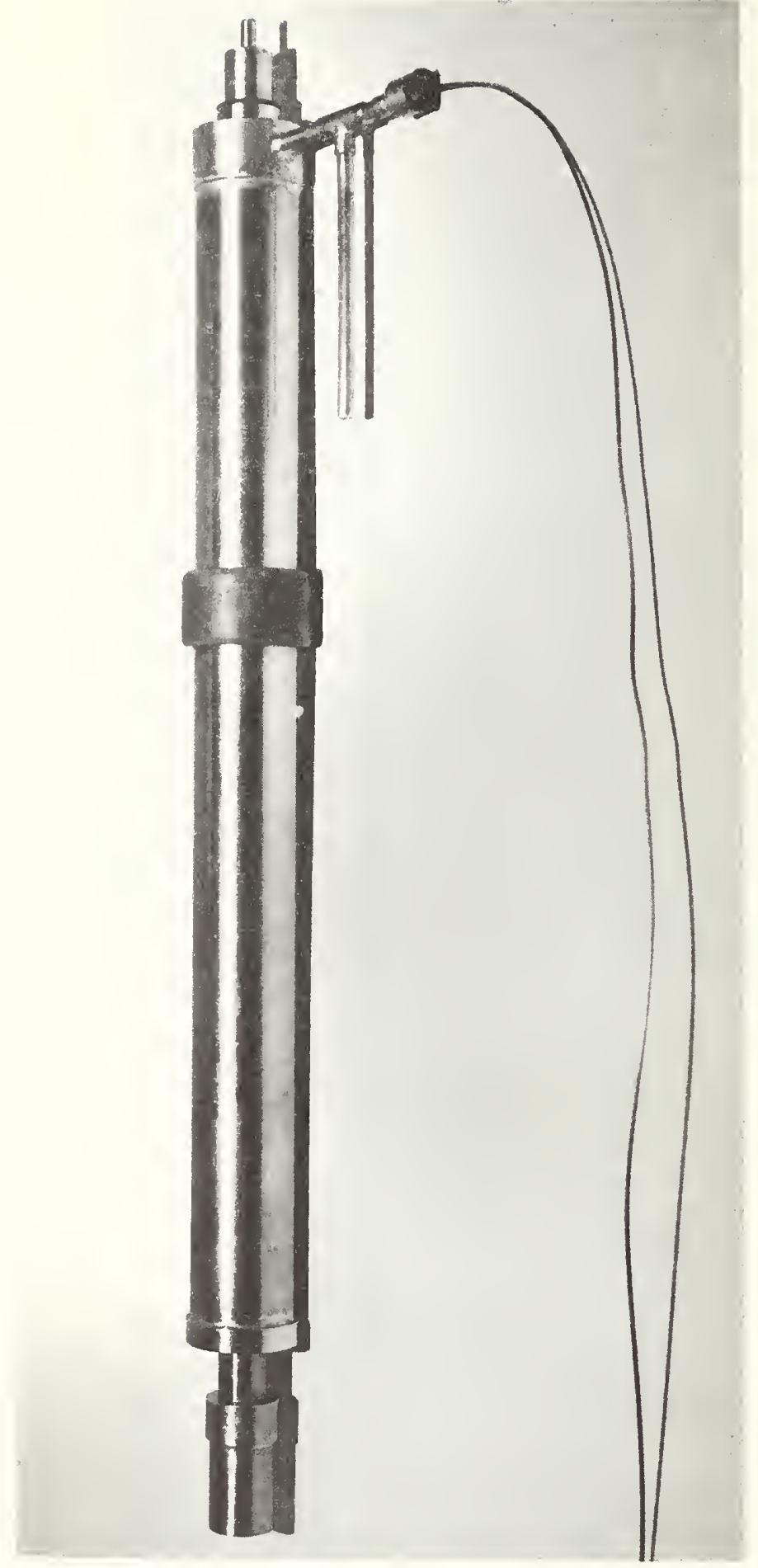

Figure 3.4 Assembled pothead. 


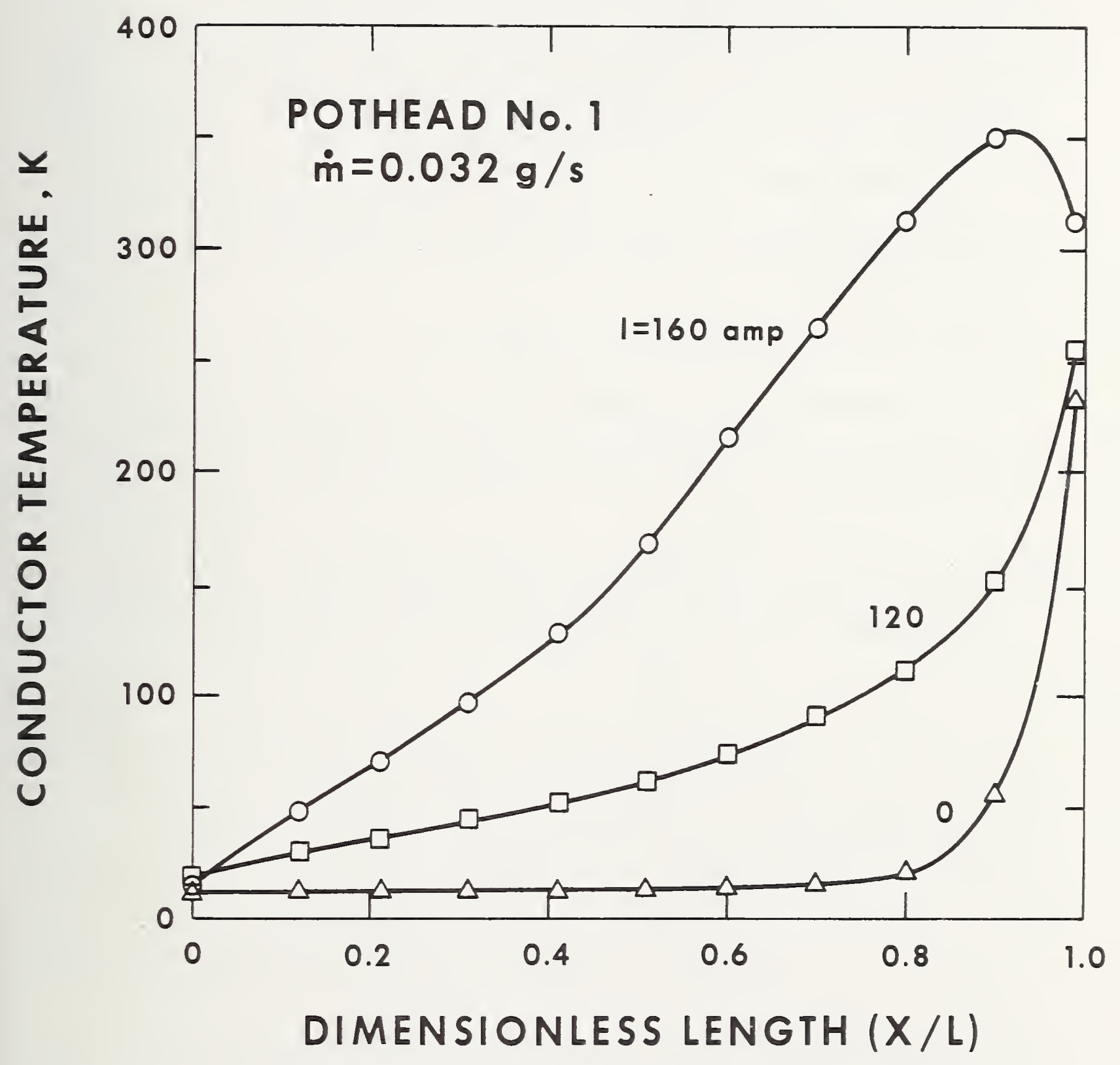

Figure 3.5 Pothead temperature profiles. 


\subsection{Nomenclature}

A conductor cross sectional area

$\mathrm{C}_{\mathrm{p}}$ specific heat of the coolant

C specific heat of the conductor

h heat transfer coefficient

$\mathrm{H}$ specific enthalpy of the coolant

I current

k thermal conductivity of the conductor

\& total conductor length

m coolant mass flow rate

p pressure*

$\mathrm{P} \quad$ wetted perimeter*

t time

T absolute temperature of the conductor

u coolant velocity

$x$ distance along the conductor.

Greek

p DC resistivity

$\rho * \quad A C$ resistivity

$\theta \quad$ absolute temperature of the coolant.

\subsection{References}

1. Clement, J. R. and Gaffney, J., Thermal oscillations in low temperature apparatus, Adv. in Cryogenic Eng., 1, p. 302 (1953).

2. Keesom, W. H., Helium, E1sevier, p. 174, (1942).

3. Scott, R. B., Cryogenic Engineering, Van Nostrans, p. 247, (1959).

4. Bannister, J. D., Spontaneous pressure oscillations in tubes connecting liquid helium reservoirs to $300 \mathrm{~K}$ environments, Proceedings IIR Commission I Meeting, Boulder, Colo. (1966).

5. Jones, M. C. and Peterson, R. G., A study of flow stability in helium cooling systems, ASME paper No. 74-WA/HT-24 (1974).

6. Efferson, K. R., Helium vapor cooled current leads, Rev. Sci. Instr., 38, 12, p. 1776 (1967).

* Note this nomenclature differs from that in Appendix A. 


\section{HELIUM PRESSURE MEASUREMENTS IN SUPERCONDUCTING POWERLINES}

Pressure measurements in helium cooling systems may be accomplished in either of two ways, (1) by bringing pressure taps out from the low temperature region to room temperature, where conventional instrumentation may be used, or (2) by placing a transducer at low temperature, in the helium system, and bringing an electrical signal out to room temperature. It is common to experience difficulties with method (1), e.g., the temperature gradients in the pressure taps promote thermoacoustic oscillations, variable thermal gradients show up as a drift in the output, and lengthy pressure lines may easily reduce the frequency response to below acceptable limits. The problem with method (2) is that no commercial instrumentation is known which can do the job in the presence of power line fields, and with a large separation between transducer and readout equipment. Thus, we have been asked to study and recommend helium pressure measurement systens for use on superconducting power lines.

The operational specifications for such a pressure measurement systen were laid out at a steering committee meeting on Sept. 19, 1974 as follows:

(a) Primary operating ranges of $4-20 \mathrm{~K}$ and pressures to $2 \times 10^{5} \mathrm{~Pa}$ (20 atmospheres). It should also be useable during cooldown from room temperature, though possibly at reduced accuracy.

(b) Overall accuracy of $\pm 1 \%$, with a precision of $\pm 0.3 \%$, for pressures ranging from static to $30 \mathrm{~Hz}$ pulsations (typical of thermoacoustic oscillators).

(c) Essentially unaffected by a.c. electric and magnetic fields of the cable, including both intrinsic calibration effects and eddy current heating.

(d) Measured output must be independent of spacing, up to 1 kilometer, between the transducer"and the recording equipment. Ifilivolt-level d.c. output voltages would be essentially unuseable.

(e) If necessary the output signal may ride at the power line voltage $\left(\Omega 10^{5} \mathrm{kT}\right)$ with conversion to ground potential occurring outside of the cryogenic envelope. [This part of the circuit design has not been very well specified in the superconducting power line studies to date, and we are actively seeking further input].

\subsection{Present Technology}

Pressure transducers have been used in liquid helium research [1], and IASA uses reliable pressure transducers in liquid oxygen and hydrogen spacefilight applications. A review of pressure transducers for liquid oxygen service has recently been completed by our laboratory [2]. The main requirements which separate this application for superconducting power line service from the others include maintaining a reliable calibration over long periods of time in a very "noisy" electromagnetic environment, and operating with a large separation between the transducer and external readout circuitry. A careful search was made of commercial pressure transducers approved for GSA (Government Services Administration) purchase, searching for one which in principle should work at helium temperature, have a sensitivity to detect $10^{4}$ pascals ( 1.4 psi) of differential pressure (or better), and produce a signal not easily nasked by noise. This search included the following types oi transducers:

(1) Capacitance transducers, which form one arm of an external bridge circuit. These can be very accurate in the research laboratory and have been used for helium studies (7). However, great care with electromagnetic shielding and avoidance of ground loops is required, and this would be very difficult in a power line environment, especially if conversion from $10^{5} \mathrm{kr}$ to ground potential is required. Commercial capacitance gauges often utilize astive circuit elements (transistors) in the transducer to bypass capacitive effects in the leads, but these would not work at low temperature. We have not found any gauges of this type which seem suitable. 
(2) Transducers using strain-gage sensing elements (often connected in a low temperature bridge circuit). These can be compact and reasonably accurate, at least for higher gage pressures, but it is customarily necessary to measure output signals to millivolt accuracy or better. This would be very difficult with long leads in the presence of powerline noise. Differential pressure gauges of this type, as would be useable for venturi flow measurement, are much less common. We have not found any commercial gauges of this type which seem suitable.

(3) Transducers employing a variable inductance (linearily variable differential transformer). The magnetic element in these units could be perturbed by the power line electromagnetic fields, though it is not clear what loss of accuracy would result. Operating and output voltages could be higher than with other types, thus easing the noise rejection problems. We did find one commercial gauge of this type, available in sensitive differential pressure form, operating at $5000 \mathrm{~Hz}$, whose design showed no obvious inconsistency with liquid helium service. According to the manufacturer, their transducer had not been used in liquid helium to their knowledge, but one of their models had been operated in liquid nitrogen at NAL. As it seemed to be the most promising commercial gauge for our purpose, we purchased one for trial. Unfortunately, one of the secondary coils failed when cooled (slowly) to $76 \mathrm{~K}$, probably because of a rigid, low expansion material used in the coil construction. We have discontinued this study.

\subsection{Possibility of Thermodynamic Pressure Measurement}

The pressure in a fluid is a function of two independent parameters, generally taken as the density $\rho$ and temperature $T$, through the equation of state $P(\rho, T)$. Since temperatures will be measured in the superconducting power line system, it is possible to calculate the pressure if one other parameter, such as density, could be independently measured. In practice, density is not the logical choice for this approach, since it is generally more difficult to measure directly than is pressure itself. However, we would be remiss if we did not consider other possibilities.

The most promising candidate, in a theoretical sense, is the velocity of sound, $c$. It can be measured with an ultrasonic technique which should work reasonably well in a power line environment. A gas chromatograph in our laboratory uses an ultrasonic transducer at the output to measure sound velocities to an accuracy $\mathrm{dc} / \mathrm{c} \approx 10^{-6}$ ( $1 \mathrm{ppm}$ ), and it is reasonable to assume that this accuracy could as well be approached in a low temperature transducer. Thus, by measuring $\mathrm{c}$ and $\mathrm{T}$, and feeding this information into an equation of state $\mathrm{P}(\mathrm{c}, \mathrm{T})$ (which would have to be developed), one could calculate the pressure in the system.

A basic limitation on this approach can be deduced from the data in figure 4.1, which gives

$$
\mathrm{c}, \quad(\mathrm{P} / \mathrm{c})(\partial \mathrm{c} / \partial \mathrm{P})_{\mathrm{T}}, \quad \text { and } \quad(\mathrm{T} / \mathrm{c})(\partial \mathrm{c} / \partial \mathrm{T})_{\mathrm{P}}
$$

as a function of pressure and temperature. One can see that the sound velocity is almost independent of pressure in the ideal gas range, and goes through a minimum along the transposed critical line, so that the useable range for a meter of this type would only be the dense fluid range, to the left of the transposed critical line in the P-T plane, i.e., in the operating range for $\mathrm{Nb}$ power lines. Within this range, the data indicate that 0.1 to $1 \%$ accuracy in $c$, combined with 0.5 to $5 \%$ accuracy in $\mathrm{T}$, would determine $\mathrm{P}$ to $1 \%$. Thus, though reasonable accuracy would be attainable in its dense fluid operating range, it would not be useable during cooldown or for operation near the transposed critical line, e.g., for $\mathrm{Nb}_{3} \mathrm{Sn}$ power lines.

We conclude that this is not a practical approach for superconducting power 1 ines, though it may be for other liquid helium applications.

\subsection{Microwave Pressure Transducer}

C. T. Van Degrift has shown that a microwave cavity can be a very sensitive displacement transducer, and could be the basis for a pressure transducer [3]. Potential operational advantages for use with superconducting power lines include the following: 


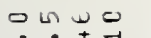
¿ N N $N \stackrel{\sim}{N}=\dot{0}$

OMN - in t i o a a anche no

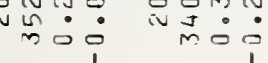

$=\infty N$

- ingaram

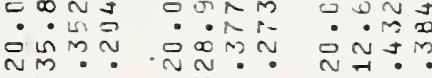

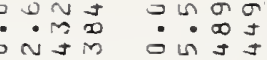

兄望:

n $\dot{0} \dot{0}$

$0+\infty 0$ $\therefore N$ - ing तें

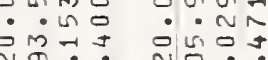
000

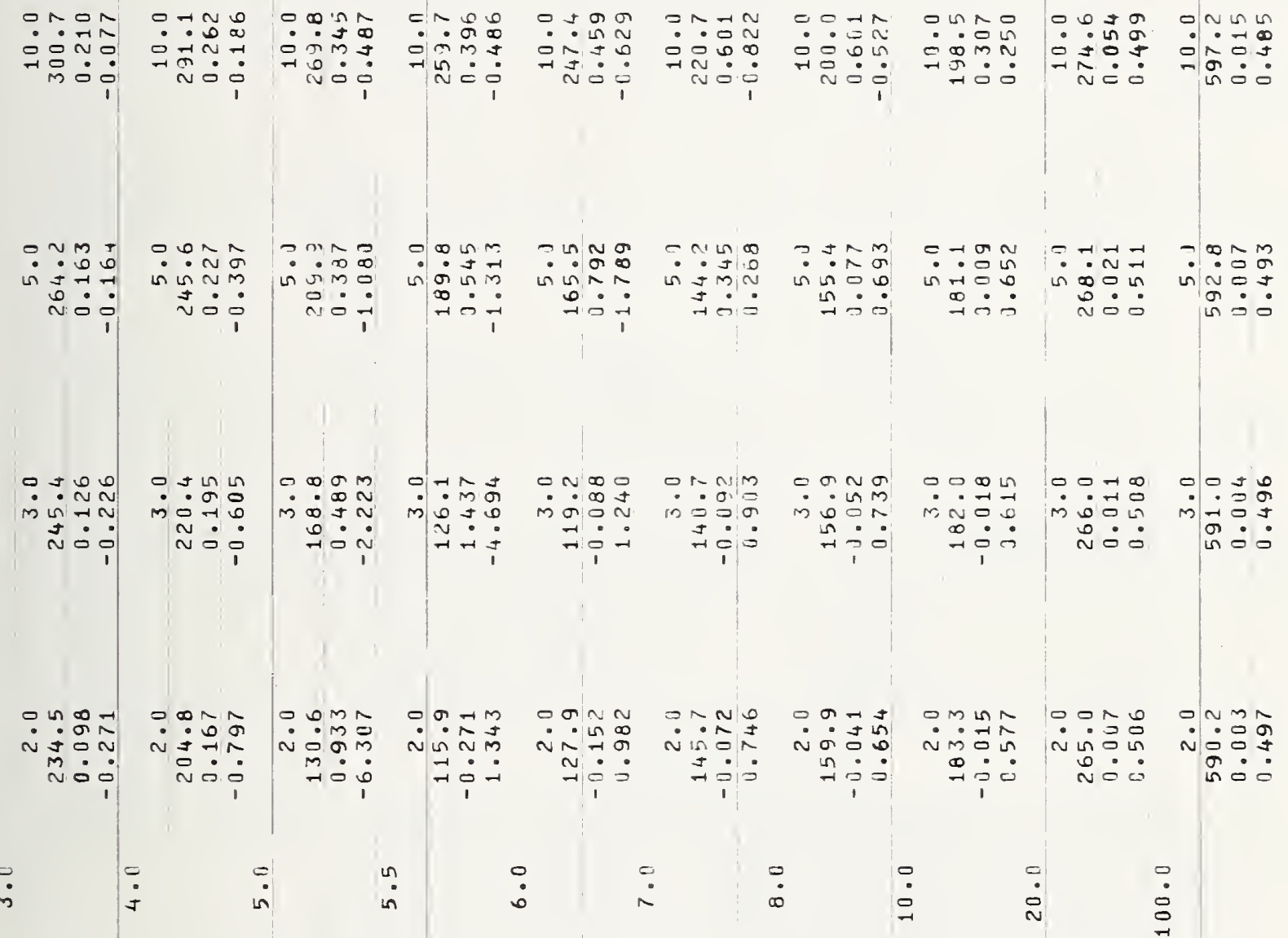

$\|$ II

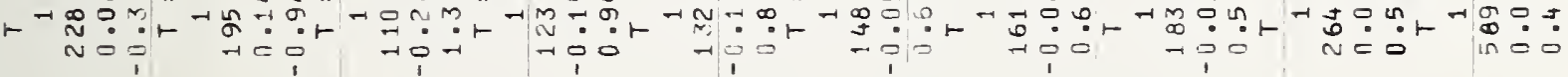

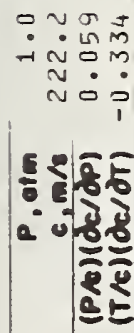

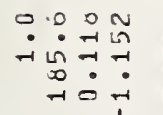

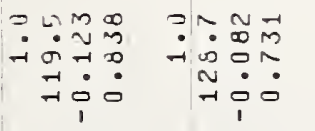
$0 \infty \infty=$
$\rightarrow \dot{0}: \hat{0} \approx$
$\rightarrow \dot{0}$

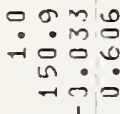

o n $=N$

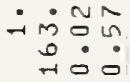

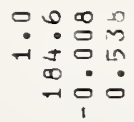

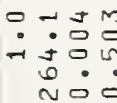

- $m=\infty$

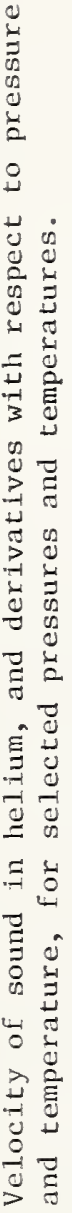

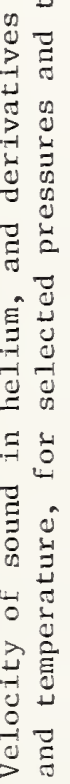

-1
$\vdots$
0
0
0 
(a) It can all be made of one metal, thus obtaining a rigid and robust structure and avoiding the differential thermal contractions associated with composite metal and insulator structures. Slow drifts in the output signal (for a constant pressure input) should be completely negligible at operating temperatures below about 20 kelvin. Also, the design should accommodate an order-of-magnitude overpressure without damage or subsequent calibration shift, by allowing the flexible diaphragm to contact and be supported by a closely spaced rigid wall.

(b) It can be made in a self-shielding geometry, so that its operating frequency is essentially unaffected by external electromagnetic fields or magnetic materials.

(c) The output signal is a frequency, typically $10^{8}$ to $10^{9} \mathrm{~Hz}$, which can be transmitted over long distances and easily filtered from $60 \mathrm{~Hz}$ "noise". Also, using radio techniques, it can be transmitted from high voltage to ground potential, e.g., $345 \mathrm{kV}$ to ground, with comparative ease.

(d) When constructed of a highly conducting material (e.g., copper) and operated at helium temperature, the $Q$ of the cavity should be very high, $\approx 10^{4}$, thus permitting high resolution to be obtained. If the operating temperature is low enough that a superconducting cavity could be used $(T \lesssim 7 \mathrm{~K}), \mathrm{Q}^{\prime} \mathrm{s}$ of $10^{5}$ or $10^{6}$ may be obtainable.

\subsubsection{Schematic Pressure-Measurement System}

The basic transducer consists of a microwave cavity having one thin wall which deflects elastically with pressure. The wall deflection causes a change in the natural resonant frequency of the cavity, which is monitored by external circuitry, as illustrated in figure 4.2 .

The important consideration in the circuit design is that the measured resonant frequency must be unaffected by the coaxial cable, perhaps 1 kilometer long, connecting the cavity to the external instruments (shown in figure 4.2 as the length 2-3). At the microwave frequencies being used, the important parameters of the coaxial cable for this application are:

(a) The characteristic impedance, $Z_{0}$, which must be matched at the termination to the cavity; mismatch, or sharp variations in $Z_{0}$ with position (e.g., due to temperature gradients), will degrade the sensitivity but have negligible effect on the calibration.

(b) Total cable length, $\ell$, which introduces a phase lag into the sensing system and reduces the high frequency response of the system. In practice, a frequency response up to $1000 \mathrm{~Hz}$ should be attainable using several kilometers of coaxial cable, so that this is not a significant limitation, and

(c) the total attenuation, $\alpha$, in the coaxial cable. If the attenuated signal is less than the noise at the amplifier input (4), feedback is lost and the system fails to produce a meaningful output; this length limitation is probably several tens of meters if the coaxial cable is normally conducting (it is quite system-dependent and difficult to estimate accurately) and will be determined experimentally. A 250 meter length of superconducting coaxial cable has been obtained from Furakawa Electric Company in Japan for testing with our cavities. Its attenuation of high frequency signals is of the order $1 \mathrm{~dB} / \mathrm{GHz} / \mathrm{kilometer,} \mathrm{which} \mathrm{is} \mathrm{so} \mathrm{low} \mathrm{that} \mathrm{cable} \mathrm{lengths} \mathrm{of} \mathrm{many} \mathrm{kilometers} \mathrm{seem} \mathrm{feasible.}$

\subsubsection{Cavity Tests}

There are two possible cavity configurations which seem attractive for this application. The first one which we tried is shown in figure 4.3, and consists of a copper cavity soldered coaxially onto a pipe containing helium. Slight deflections of a thin wall section of the pipe cause capacitance changes to a close-fitting annular piece. Experimentation with this cavity, which resonated at about $440 \mathrm{MHz}$, led to problems of difficult assembly and disassembly, and mechanical construction in general. The advantage of this geometry is that a suitably shaped central channel could probably be used to construct a venturi flowmeter with the same microwave circuit. However, numerous small problems led to the conclusion that this was just not a good choice for initial experimentation, where ease of assembly is important. 


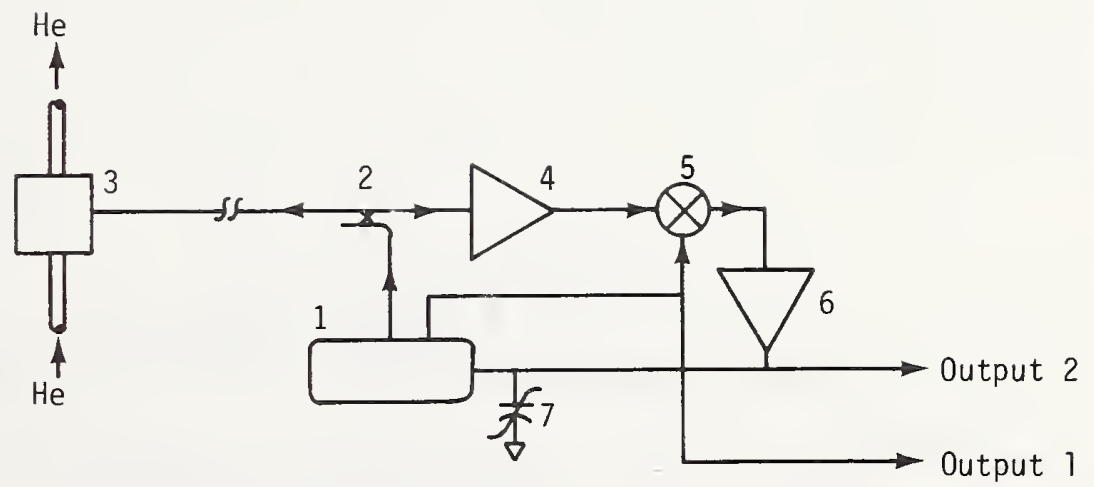

1. UHF Oscillator (Tracking)

2. Directional Coupler

5. Mixer

3. Sensor (Resonant Cavity)

4. Amplifier

6. Amplifier

7. Varactor Tuner for the UHF Oscillator

\section{BASIC SENSOR SYSTEM}

The UHF oscillator (1) drives the sensor (3) through the directional coupler (2) and the long transmission line. The signal reflected from the sensor is amplified (4) and multiplied by the reference signal from the UHF oscillator (1). The output, which is a function of the amplitude and the phase variation of the reflected signal, is amplified (6) and used to tune the UHF oscillator (1) via the varactor (7).

Figure 4.2. UHF oscillator measurement system. 


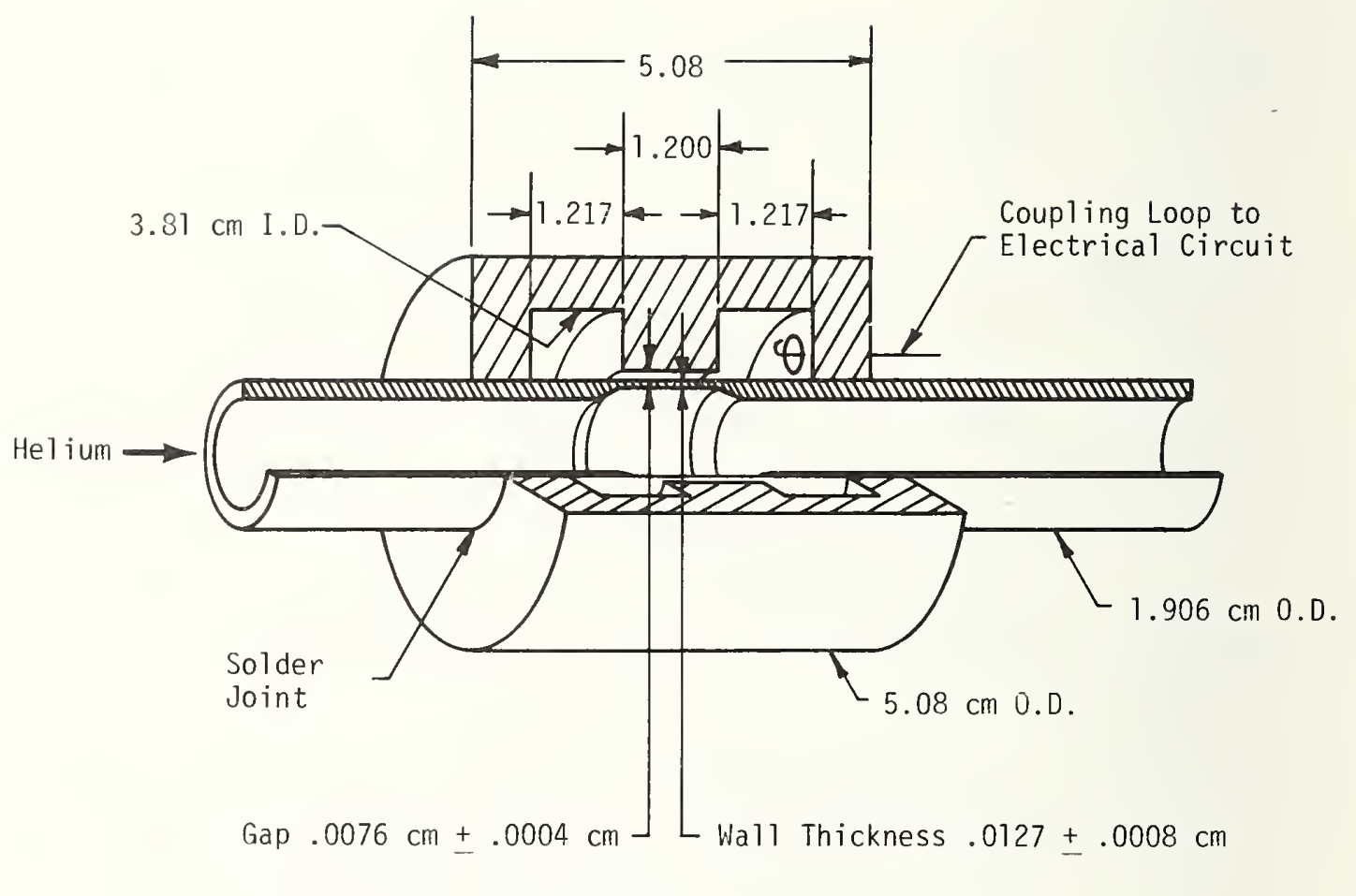

A11 dimensions in centimeters.

Figure 4.3. $440 \mathrm{MHz}$ resonant cavity pressure sensor. 
A different configuration was used in the second cavity, illustrated in figure 4.4, operating at $369 \mathrm{MHz}$. The pressure sensing diaphragm is realtively thick, $0.11 \mathrm{~cm}$, giving high stability and freedom from damage by inadvertant overpressure. The pressure sensing chamber is easily separated from the microwave cavity, and small clearances in the gap region are measured and machined with relative ease.

Experiments with this cavity have shown:

(1) The Q of the cavity is about 9000 at helium temperature, using ordinary machining techniques on copper. This means that we can resolve a diaphragm displacement of about $1.9 \times 10^{-6} \mathrm{~cm}$, or a pressure change of about $2 \times 10^{-3} \mathrm{~N} / \mathrm{cm}^{2}\left(3 \times 10^{-3} \mathrm{psi}\right)$.

(2) A simple mechanical force on the lip (where the cavity is assembled from tho parts) is not adequate to obtain a high $Q$, and some sort of soldered contact is necessary.

(3) By far the largest perturbation on system stability comes from thermally-induced distortions in the coaxial line which couples external instruments to the cavity, with resultant small variations in impedance matching as the center conductor moves slightly. To minimize this effect, we have switched from inductive to capacitive coupling between the coaxial line and the cavity body, and are making other changes in the attachment of the coaxial lead to the cavity body. Satisfaction with and final testing on an optimum lead design has not yet been done, but we are confident that this perturbation can be reduced to insignificance.

At the project review held in Boulder on May 7, 1975, some concern was expressed about the physical size of the cavities, and we were asked if they could be made smaller. To accomplish this, one is forced to operate a higher frequency where (a) Q's are lower, (b) coaxial line losses are higher, and (c) external amplifiers are more noisy. To explore this, we have built a third cavity designed to operate at $1200 \mathrm{MHz}$, as illustrated in figure 4.5. This frequency is chosen as being about the highest frequency for which suitable amplifiers can be purchased commercially (having come on the market in just the last year or so). For a given frequency, the mushroom-shaped pillar in the cavity increases the capacitance, allowing a smaller inductance (cavity volume) to be used; however, it also increases the path length over which the microwave currents must flow, and hence reduces the Q. Further, with this design, the total capacitance changes less with diaphragm displacement than, for example, in the configuration of figure 4.4. The design also incorporates a capacitive coupling probe of the type developed with the $369 \mathrm{MHz}$ cavity. The overall size reduction, from about 7 to $2 \mathrm{~cm}$ diameter is very significant. Because of a delay in obtaining a $1200 \mathrm{MHz}$ amplifier, we have not yet tested this device.

\subsubsection{Future Work}

In order to complete a logical development step, we plan to

(a) further evaluate and improve if necessary the stability of the cavity with respect to temperature changes and external mechanical stresses induced by the support mechanism.

(b) evaluate performance as a differential pressure gauge, as well as the absolute pressure gauge tests to date.

(c) Evaluate effects of long coaxial lead length.

(d) Compare the $369 \mathrm{MHz}$ cavity with the $1200 \mathrm{MHz}$ cavity for the above items (a) $-(c)$.

(e) Obtain practical experience by installing and operating one in the pothead test apparatus described elsewhere in this report.

In addition, it may be desirable to obtain higher Q's by 


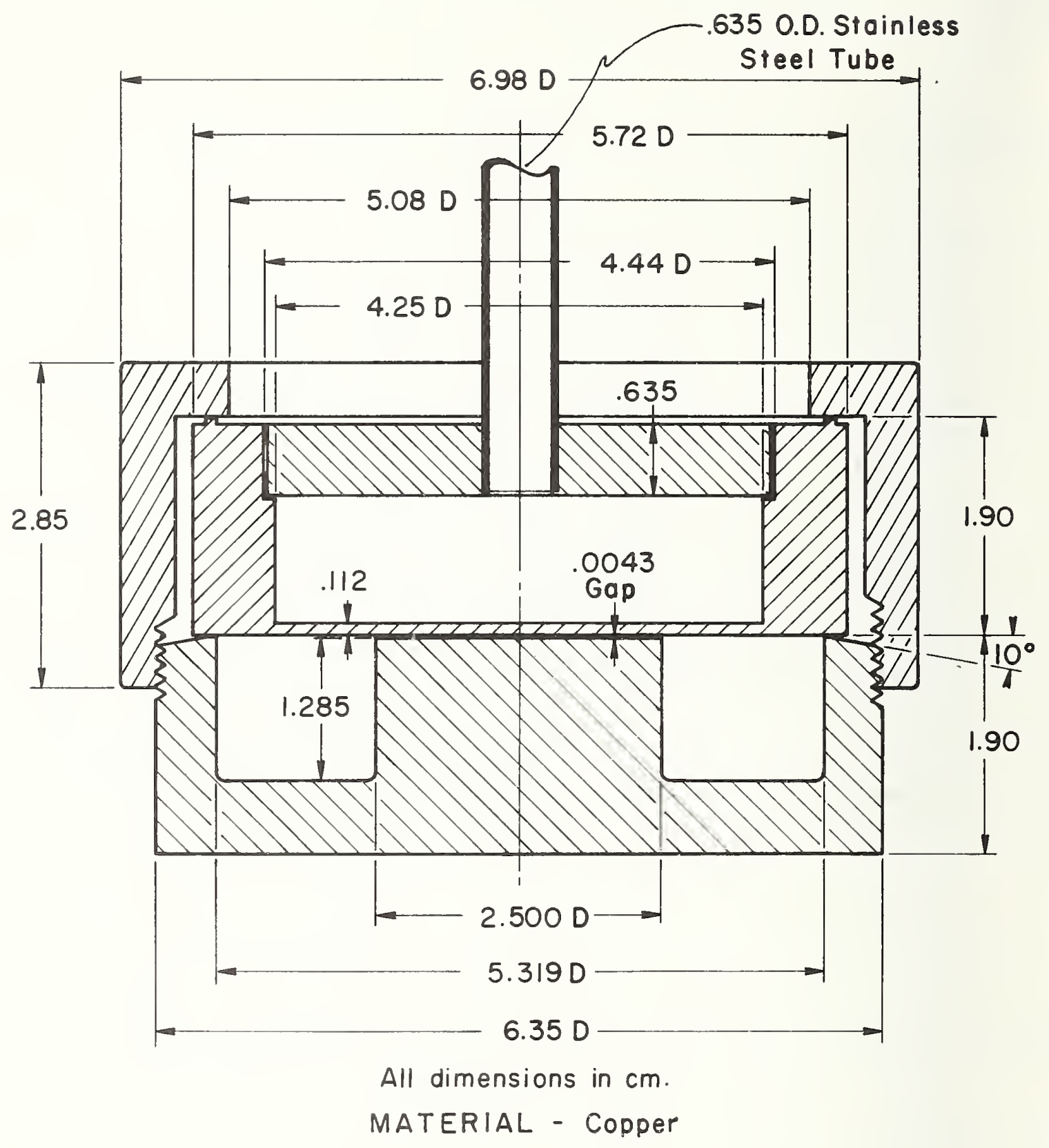

Figure 4.4. $369 \mathrm{MHz}$ resonant cavity pressure transducer. 


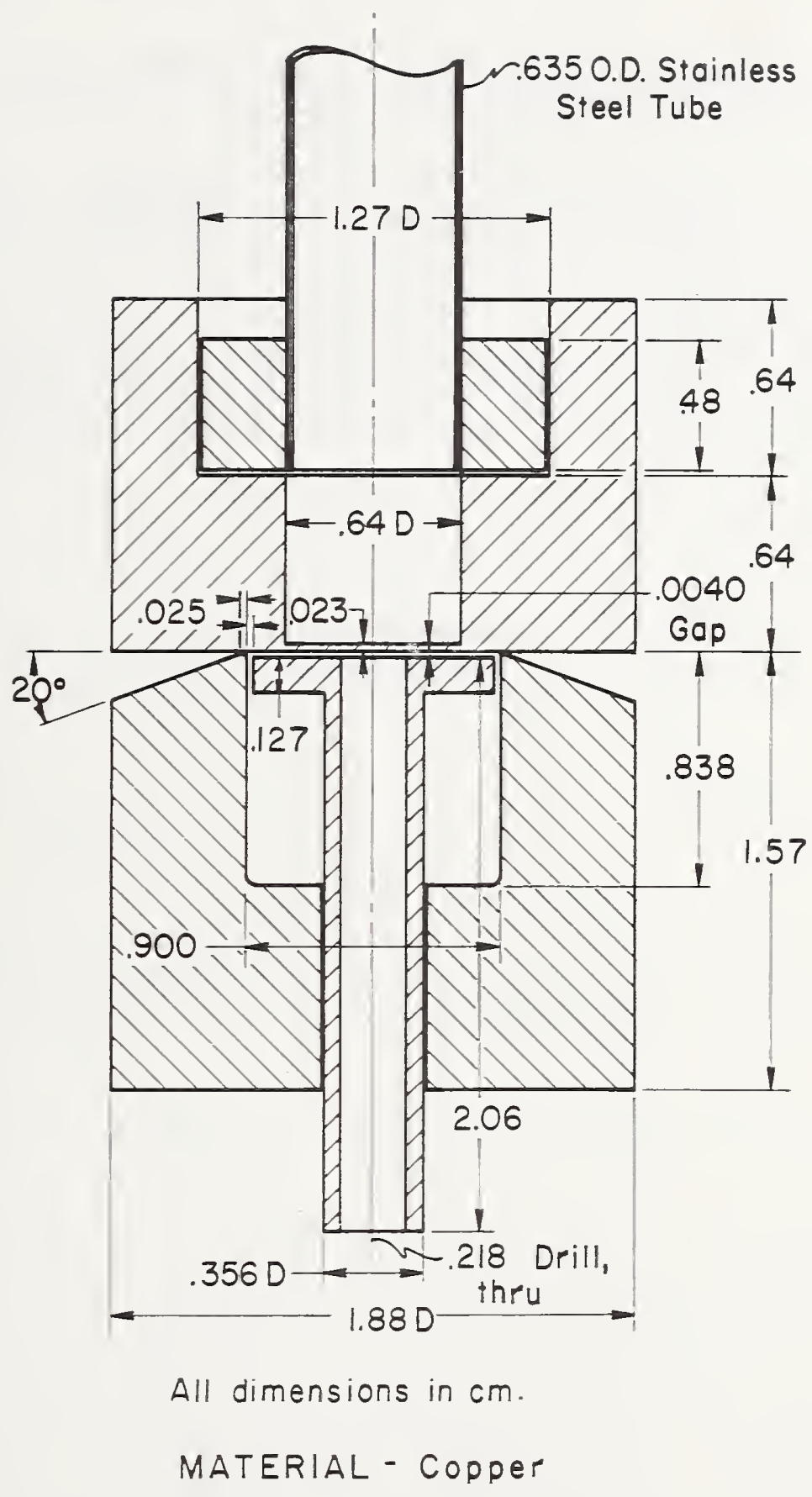

Figure 4.5. 1200 MHz resonant cavity pressure transducer. 
(f) improved surface-finishing techniques on copper cavities, or

(g) using lead-plated cavities to obtain additional loss reduction from superconductors.

These last two items are of lower priority, and will be undertaken only if the above work items suggest that it would be worth the effort.

\subsection{References}

1. Wheatley, J. C., New techniques in refrigeration and thermometry, p. 455, Low Temperature Physics-13, Plenum Press, N.Y., 1974.

2. Arvidson, J. M. and Brennan, J. A., ASRDS oxygen technology survey - pressure measurement, NASA Special Publication.

3. VanDegrift, C. T., A sensitive displacement transducer using an extremely reentrant $84 \mathrm{MHz}$ cavity oscillator, presented at the 1974 Applied Superconductivity Conference. 


\subsection{Introduction}

The goals for this part of the program were to make recomendations for reasonably accurate thermometry in superconducting transmission lines. Superconducting lines present very unusual environments for conventional thermoneters. Future designs may call for up to $700 \mathrm{kV} \mathrm{a} . c$. or d.c. For the potential difference between conductors and up to $50 \mathrm{kA}$ current along the conductors. The electric fields caused by these voltages may be of the order of $20 \mathrm{kV} / \mathrm{mm}$ with higher harmonics and ripple fields. The peak magnetic fields will typically be of the order of 0.1 tesla at the conductor surface in a.c. cables, and no more than 1 . tesla for the highest power d.c. cables under consideration. Many types of thermometers are potentially suitable for instrumentation in the two main modes of operation: 4 to $20 \mathrm{~K}$ under stable conditions or 4 to $300 \mathrm{~K}$ during cooldown, (or possibly after a serious system failure). The types of available thermometers differ radically in the physical phenomena that they measure and therefore it is expected that they will differ significantly in their response to high, alternating or static, electric and magnetic fields. The main five types of thermometers that are potentially suitable are discussed in the next section.

Thermometer measurements can be affected in three different manners by electrical and magnetic fields. First of all, the fields may directly affect the physical phenomena being measured, e.g., the magnetoresistance effect in metals or electric field gradient effect in semiconductors. Second, the thermometer casing may be heated by alternating fields. This may occur even for fluid thermometers. This effect, if carefully considered, can be minimized by good design and placement of the thermometer holders. The third effect is the disturbance of the transducer or instrumentation wire leads by alternating fields or erratic pulses in nominally static fields.

The thermometry project was divided into three phases for the preliminary studies:

1) 1iterature survey,

2) estimates of probable effects, and

3) recomendations for appropriate experimental tests.

The literature survey revealed, as expected, very little on the effects of altemating magnetic or electric fields or static electric fields. There, however, is a considerable body of information on the effects of high static magnetic fields. Thermometers that are poor in high static magnetic fields, such as some thermocouples, will of course also te poor in alternating fields.

An outline of the present knowledge on the effects of electromagnetic fields on thermometers is given in section 5.3. A sumary of the results of a literature search is given in 5.4. Further development of this program is discussed in section 5.5.

As it was stressed in the Steering Comittee meetings, it is very important that the thermonetry project reflect the true needs of the practical transmission line assemblies and that there be conplete compatibility of measurement systems and cable configurations. The thermometry project is therefore oriented toward the needs of the three main U.S. efforts on superconducting transmission lines, the programs directed by: Brookhaven National Laboratory, Linde Div. of Union Carbide, and the Los Alamos Scientific Laboratory, and funded by the Energy Research and Development Administration and the Electric Power Research Institute. Very thorough reviews of those programs have been given by Belanger $[1,2]$. Very briefly, a) the LASI line is d.c., the other two a.c., b) the BNL line is ilexible with wrapped tapes, the other two rigid or semi-rigid. These differences lead imediately to considerable complexity in designing a thermometry system that can be used for any one of the transzission lines. An additional complexity is introduced when one wishes to have both fast response time thermometers for diagnostic tests and more stable ones for operating lines. The various criteria, with some explanations and possible solutions, are discussed in section 5.4.1. 


\subsection{Types of Thermometers}

The five main types of thermometers that were investigated as potentially useful in these applications were:

1. active electrical - thermocouples,

2. passive resistive electrical - carbon, germanium or platinum resistance thermometers, semiconductor diodes, etc.

3. passive capacitive electrical - glass-ceramic capacitors,

4. magnetic - paramagnetic and nmr,

5. fluid - vapor pressure, gas pressure, or acoustic.

\subsubsection{Active Electrical-Thermocouples}

Richards, et al. [3] have shown that the thermoelectric output voltage is independent of magnetic fields fluctuations if there is no temperature gradient along the wires that are in a magnetic field. However, it is very unlikely that it would be possible to obtain those severe restrictions in a working system. Therefore the effects of electromagnetic fields on thermocouples would have to be considered carefully.

There are very accurate thermocouple calibration tables for all of the thermocouples commonly used at low temperatures [4,5,6]. However, it is well known theoretically [7], that there is usually a strong dependence of the thermoelectric voltage, E, on magnetic field. This effect can be calibrated, and since thermocouple wires are usually very small and polycrystaline, there would not be a large orientation effect.

There have been recent experiments on the magnetic field dependence of copper-constantan thermocouples [8], though they are not the preferred system for cryogenic usage [4]. There is a positive temperature error of about 2 percent at $20 \mathrm{~K}$, less at higher temperatures. At lower temperatures that couple has so little output that its signal would be insensitive to thermal fluctuations and possibly masked by electromagnetic noise in the measuring system.

There is also some data on Au-Fe elements reported by Berman and colleagues [9,10]. The effects are quite complex: at low temperatures (near $1 \mathrm{~K}$ ) the effect is positive up to $2.5 \mathrm{~T}$, then negative at higher fields; at higher temperatures the increase in thermoelectric power is monotonic. Sample, et al. [11] have recently measured the effects of fields on KP vs Au-Fe and TN couples. They show that it is possible to obtain reliable correction factors for well controlled wires in laboratory systems.

In summary, the effects of electromagnetic fields on thermocouples are quite complex, calibrations and corrections would be quite difficult to carry out in practice for field installations.

\subsubsection{Passive Resistive Electrical}

Platinum resistance thermometers are the most often used accurate thermometers in much of the whole temperature range. Their properties and methods of utilization have been well described by an NBS-Gaithersburg group [13]. However, they have a rapidly decreasing temperature sensitivity at low temperatures making them a poor choice below about $12 \mathrm{~K}$. They also, unfortunately, have a relatively large magnetoresistance. Platinum thermometers are constructed of high-purity platinum and so their electrical resistance is governed primarily by scattering from phonons. This scattering mechanism can be highly affected by electromagnetic fields and it becomes more severe at low temperatures. Curves derived from the work of Neuringer and Shapira [14] are given in the next section.

Germanium resistance thermometers are also widely used for accurate thermometry at low temperatures. Like platinum thermometers, they also have a large magnetoresistance effect. Germanium thermometers are fabricated from single crystals of doped, but moderately pure, material. Because of the technological refinements in semiconductor manufacture, they can be made both reproducible and temperature sensitive. In fact, they have become the best accurate thermometer in their main temperature range from 2 to $20 \mathrm{~K}$. Because of the two characteristics of germanium crystals, their sensitivity and crystallinity, they have 
both a large magnetoresistance and a significant orientation effect [15]. Values for these effects are given in the next section.

Because of these characteristics, germanium thermometers are not recommended for use in practical transmission cables in magnetic fields above 0.1 tesla. However, after careful calibration, they may be used as reference thermometers in laboratory systems at higher fields.

Semiconductor junction diodes have recently entered the competition as reliable lowtemperature thermometers. Their main advantage is their very wide useful temperature range, from about 1 to $300 \mathrm{~K}$. They are also quite easy to measure electrically. The diodes utilize the phenomenon that the forward voltage drop of the junction increases with decreasing temperature if the forward current is kept constant. The voltage is current sensitive and the polarity of measurement is critical, therefore the current polarity on the leads must be kept proper and the current must be amplitude regulated (nominally $10 \mu \mathrm{A}$ ). These are, however, not serious difficulties in instrumentation systems. The Swartz's [16] have recently written a review on diode thermometers (as well as on resistance and capacitance thermometers). The $\mathrm{p}-\mathrm{n}$ diode junctions may be constructed from silicon $[17,18]$, germanium [19], or GaAs $[20,21,16]$, but only the latter is both available commercially and has had extensive testing. The effects of magnetic fields have been determined for some types of $\mathrm{Ga}$ As diodes [22] and they are reviewed in the next section. Using optimum conditions of bias current and orientation for the best units, the percentage error in temperature can be made very small.

Because of their favorable characteristics, GaAs or Si p-n junction diodes appear to be one of better possibilities for thermometry in superconducting transmission lines.

Thermistors are also relatively new in low temperature thermometry. The first discussion of their characteristics was given by Schlosser and Munnings [23]. However, they have not received the recognition accorded other thermometers. They usually have a extremely high temperature sensitivity, but it occurs only over a narrow temperature range. The same authors have determined the effects of magnetic fields on various types of thermistors -- the effect is usually very small [24]. However, because of the lack of general usage, the necessity of using different types in different temperature ranges, and the sometimes-observed anamolous effects, they are not generally recommended for measurements in transmission lines.

Carbon resistance thermometers are very often used as secondary sensors in cryogenic systems with high magnetic fields $[11,12,14,25,26]$. They have a good temperature sensitivity, good reproducibility (if handled properly), and small size. Because of the small size they also have a small heat capacity. Fortunately the magnetoresistance is both small and very reproducible so that the units can be accurately calibrated without too much effort. They also show a negligible orientation effect.

Several different sizes of carbon resistors have been tested as thermometers. The $47 \mathrm{ohm}, 1 / 4$ watt ones from one well-known manufacturer seem to give the best compromise of temperature sensitivity, reproducibility, and magnetic field dependence.

Results on the magnetoresistance of several sizes of carbon resistors are given in the next section.

Because of their excellent characteristics, carbon resistors (when specially selected and handled) would seem to give one of the best thermometry systems available for diagnostic tests. They can probably also be used in practical transmission lines, especially if they have been well encapsulated and calibrated.

Another of the newer thermometers is the carbon-glass (carbon-impregnated porous glass) resistor developed by Lawless [27]. The materials, when encapsulated, are extremely reproducible, mechanically strong, and interchangeable. They are constructed from a phase-separated alkali-borosilicate glass that has been leached to remove the boron-rich phase. The porous glass is saturated with a high-purity organic fluid which is then decomposed thermally. This process leads to filamentary carbon conductors embedded in 
the porous glass matrix. Because of the lack of clay filler (as with carbon radio resistors) and the high purity of the carbon, the units are very homogeneous and interchangeable. Their resistance ratios are high (e.g., $\left.\mathrm{R}_{4.2} / \mathrm{R}_{300} \approx 130\right)$ but their room temperature resistivities are low (e.g., $\left.\rho_{300} \approx 0.7 \Omega \mathrm{cm}\right)$. Recently Swartz, et al. [28] have measured the magnetoresistive effects in these thermometers and the results are given in the next section. They show no significant orientation effect [28].

\subsubsection{Passive Capacitive Electrical}

The glass-ceramic capacitive thermometer developed by Lawless $[29,30]$ has many advantages that will suit it very well for instrumentation in a transmission line: 1) It is sensitive, fast in response, and relatively small; 2) it can have a simple electrical readout, either capacitive variation, or with special circuitry, frequency variation; 3) one unit can give an unusually sensitive output over the entire cryogenic range; and 4) a virtually field independent output signal. However, it does suffer from two disadvantages, 1) The capacitance goes through a maximum between room temperature and helium temperature (typically at about $80 \mathrm{~K}$ ), so that its output is not a unique function of temperature during cooldown.

2) They are occasionally beset with small calibration drifts, $\approx 10^{-2} \mathrm{~K}$, whose origin is not completely understood at this time. The $\mathrm{SrTiO}_{3}$ capacitor is fabricated by forming a alumino silicate glass from a melt of SrO and $\mathrm{TiO}_{2}$ refractory oxides dissolved in $\mathrm{SiO}_{2}$ with some $\mathrm{Al}_{2} \mathrm{O}_{3}$ additive. If the melt is rapidly quenched, a stable glass-ceramic $\mathrm{SrTiO}_{3}$ is formed after the material is reheated and recrystallized. The dielectric permittivity of this glass is strongly temperature dependent at low temperatures. The composite capacitor consists of 25 layers, $0.025 \mathrm{~mm}$ thick, of glass with 26 layers of Au-Pt separating them. A platinum can is used for encapsulation. A description of the characteristic properties of commercial units has been given recently by the Swartz's [16]. In fields up to 18 tesla, deviations are less than 1 millikelvin [31].

In sumary, the $\mathrm{SrTiO}_{3}$ glass-ceramic thermometer is potentially, very promising for use in transmission line thermometry; its output may be read and processed in several ways and it has a negligible magnetic effect. Commercial units are now available for the simple kind; intermixed types would have to be fabricated specially.

\subsubsection{Magnetic}

Various paramagnetic and nmr thermometers are available for cryogenic use. The thermometers are too complex in application, often too large, and too sensitive to electromagnetic fields to be useful for practical iransmission lines. Rubin [25] has reviewed their characteristics and properties.

\subsubsection{Fluid}

Gas thermometers can be quite accurate, but they are slow in response, often bulky, and difficult to instrument conveniently. Because of their fundamental accuracy, they are often used as a reference thermometer. However, they are not suitable, in general, for practical systems with confined space or long transducer lines.

Vapor pressure thermometers are extremely sensitive and accurate in their applicable temperature ranges. However, there is no available thermometric fluid for the main temperature ranges of interest for transmission lines, 5-15 kelvin. Therefore they are also not suitable for use in this application.

The other main fluid type thermometer is the acoustical one. It has been used to develop the basic standard germanium resistance thermometer scale, but its measurement system is too complex for use in anything but basic standard thermometry.

A brief review of these three fluid thermometers has been given by Rubin [25]. 


\subsection{Effects of Electromagnetic Fields}

An exhaustive literature search was performed by our Cryogenic Data Center [32] on thermometers in general, and the effects of electromagnetic fields in particular. As expected, the literature search turned up almost nothing relating to effects of a.c. or d.c. electric fields on thermometers useful for transmission lines. Several references of one sort or another were found. Most of them referred simply to problems of measuring resistance thermometers with a.c., rather than d.c., potentiometers or circuits.

There were the same number of references on the effects of magnetic fields, but most of them were pertinent to this thermometry project. Those references have been cited in the previous section and several will be referred to again in the following pages.

First will be shown the magnetoresistance of two types of thermometers that are not likely to be used in practical transmission lines because they do have a large effect. In figure 5.1 are some semi-quantitative results by Neuringer and Shapira [14] on platinum thermometers with the magnetic field parallel to the length of the enclosing capsule. The magnetoresistance ratio is large and temperature dependent. Temperature errors, as shown in Table 5.1, would be several percent at fields of 1 tesla.

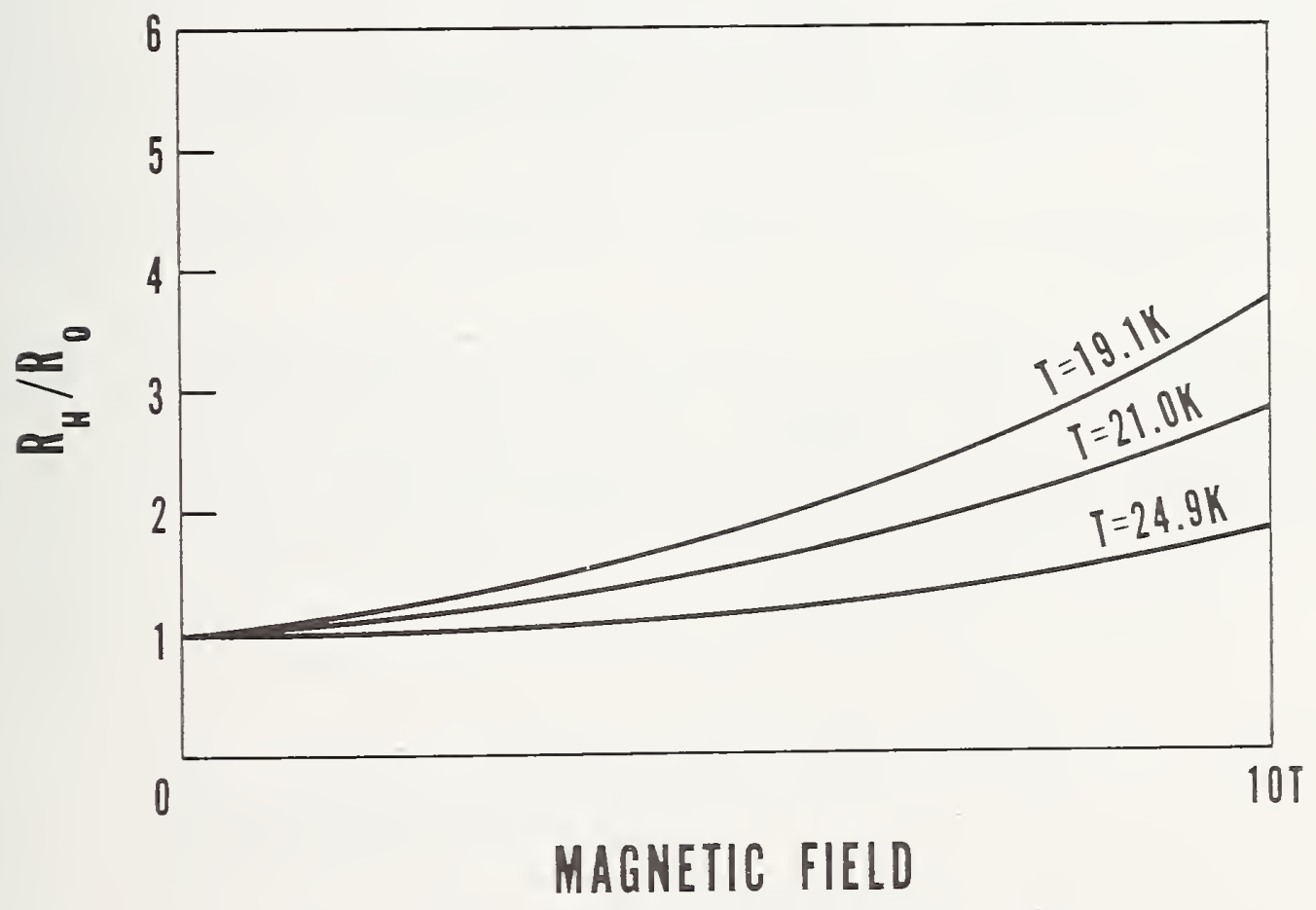

Figure 5.1. Magnetoresistance of platinum resistors. 
TABLE 5.1

Type of thermometer

Platinum

Germanium

Thermistor

Carbon resistor

Carbon impregnated glass

GaAs diode
Temperature

4. $2-20$

4. $2-20$

4. $2-20$

4.2

4.2

10

$\mathrm{SrTiO}_{3}$
$\Delta \mathrm{T} / \mathrm{T}(\%)(0-2.5 \mathrm{~T})$

$$
5-30
$$

$<0.05$

$<1$

$2-3$

$1.5-2$

$0.5-1$

not measurable

The second set of curves, figure 5.2, is on germanium with the magnetic field perpendicular to the current and long side of the encapsulation. The results are from Neuringer, et a1. [15]. Figure 5.3, adopted from the same publication, gives similar curves showing the orientation effect. In fields of 1 tesla, germanium thermometers show a magnetoresistive effect of several percent, decreasing with increasing temperature. A typical result is given in Table 5.1 .

The magnetoresistive effects in carbon thermometers are considerably smaller [14]. A graph of the effects for $47 \Omega, 1 / 4 \mathrm{~W}$ Allen-Bradley resistors is given in figure 5.4 . For those measurements, the magnetic field was perpendicular to the current in the resistor. The equivalent curves showing the effect of temperature at different fixed fields are given in figure 5.5. Note that in both curves the magnetoresistance is expressed as a percent change in the resistance. The tabular summary is given in Table 5.1.

The magnetoresistive effect in carbon-impregnated glass is not very large, though it is about twice that of carbon radio resistors. A curve derived from Lawless' research [27] is given in figure 5.6 .

Summarized results, modified from the ones given by Neuringer and Rubin [26], are given in Table 5.1, for maximum magnetic fields of 2.5 tesla. Since magnetoresistance is generally proportional to the square of the magnetic field, the temperature errors at a peak field of 0.1 tesla will be about $1 / 625$ of the 1isted values; at a peak field of 1 tesla, they will be about $1 / 6$ of the listed values. On this basis, one may conclude that all thermometers listed in Table 5.1 would be acceptable in peak fields of 0.1 tesla, though platinum and germanium thermometers would be only marginally so, becoming unacceptible at the higher fields. 


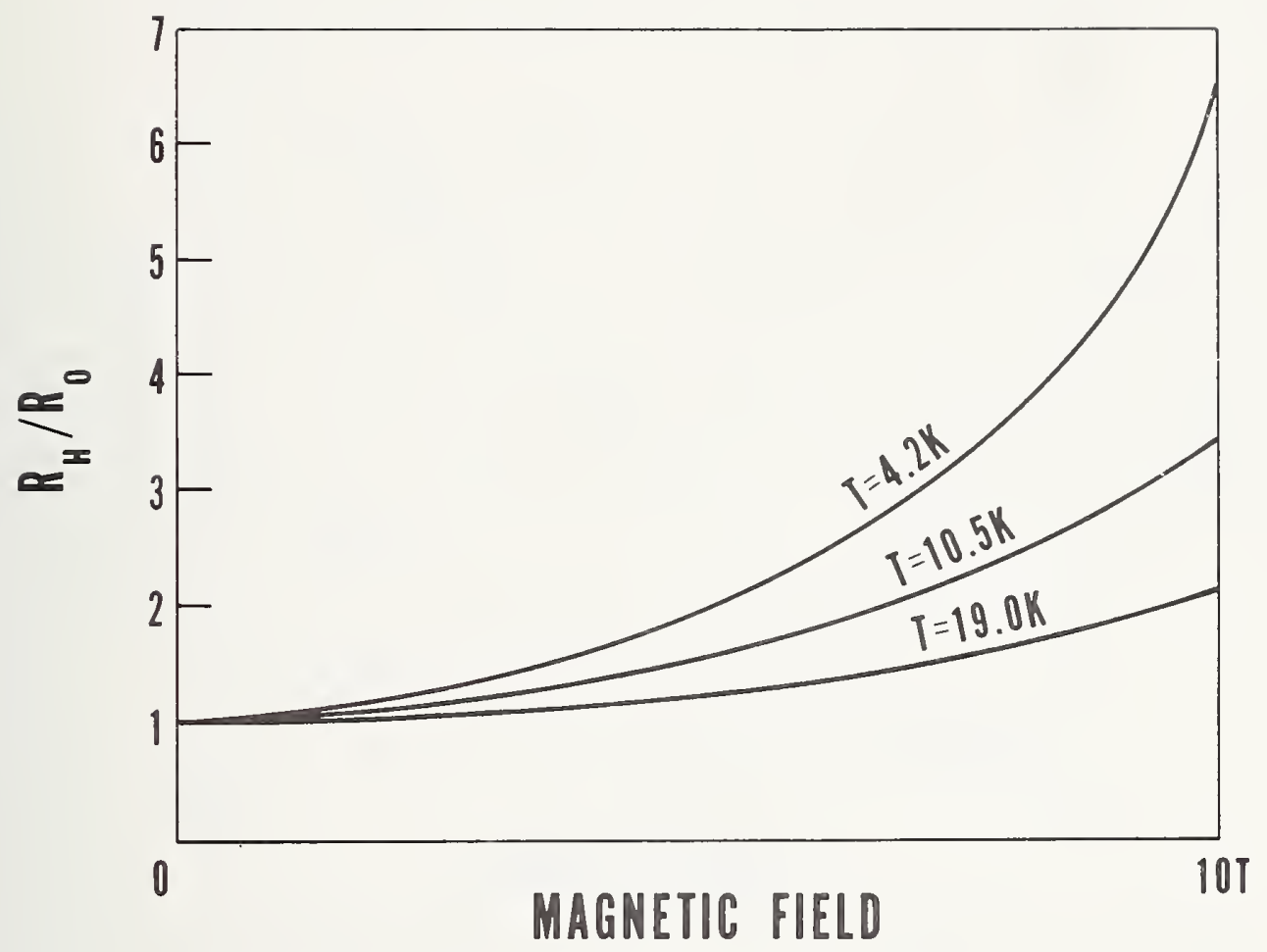

Figure 5.2. Magnetoresistance of germanium thermometers.

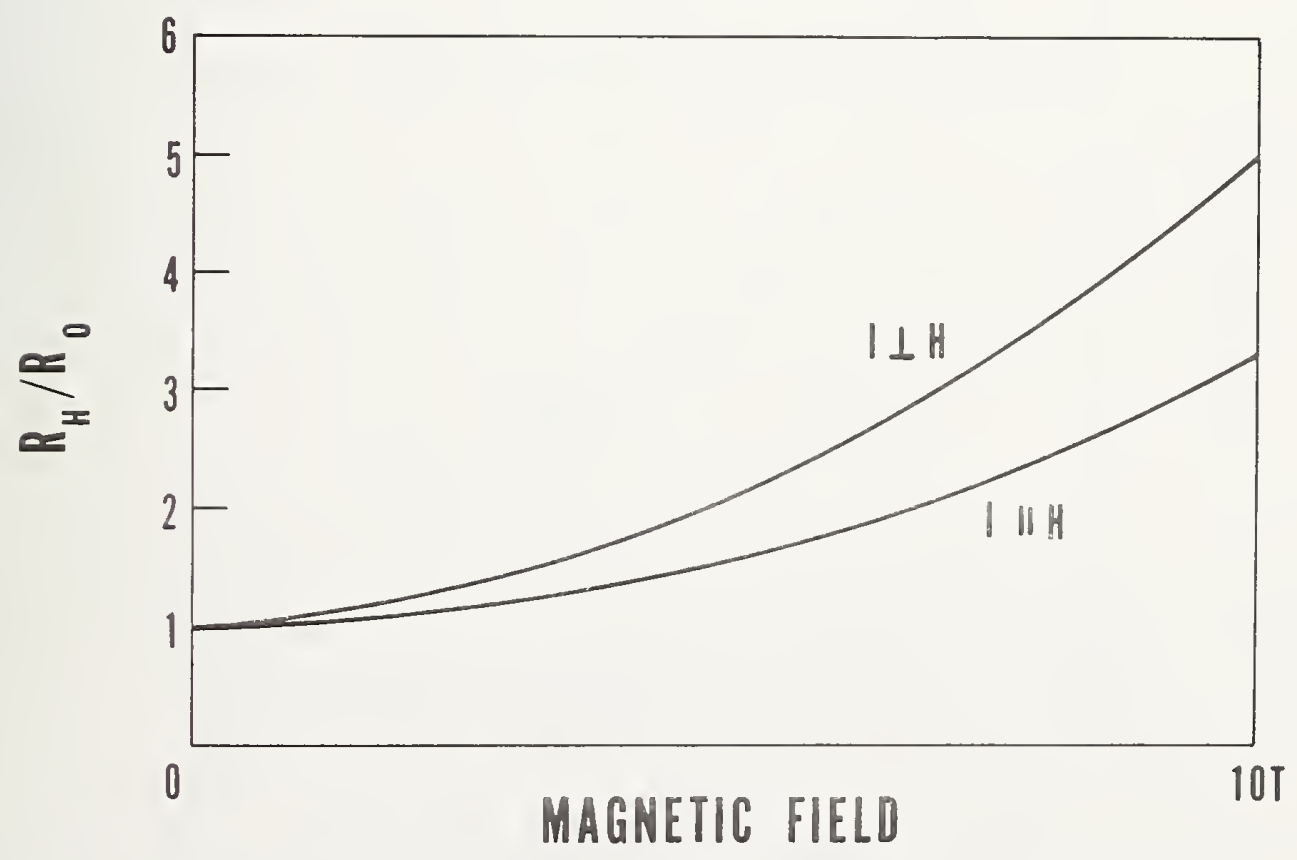

Figure 5.3. Magnetoresistance of germanium thermometers. 


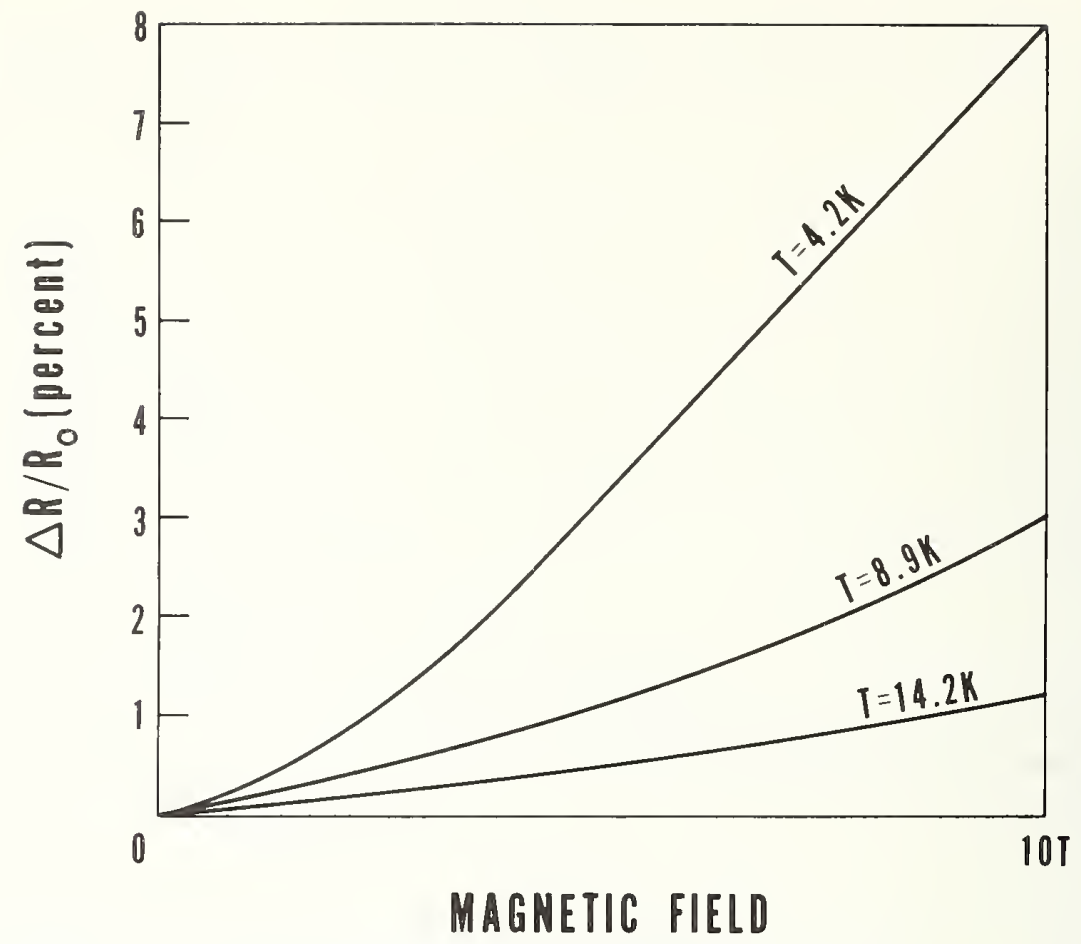

Figure 5.4. Magnetoresistance of carbon resistor thermometers.

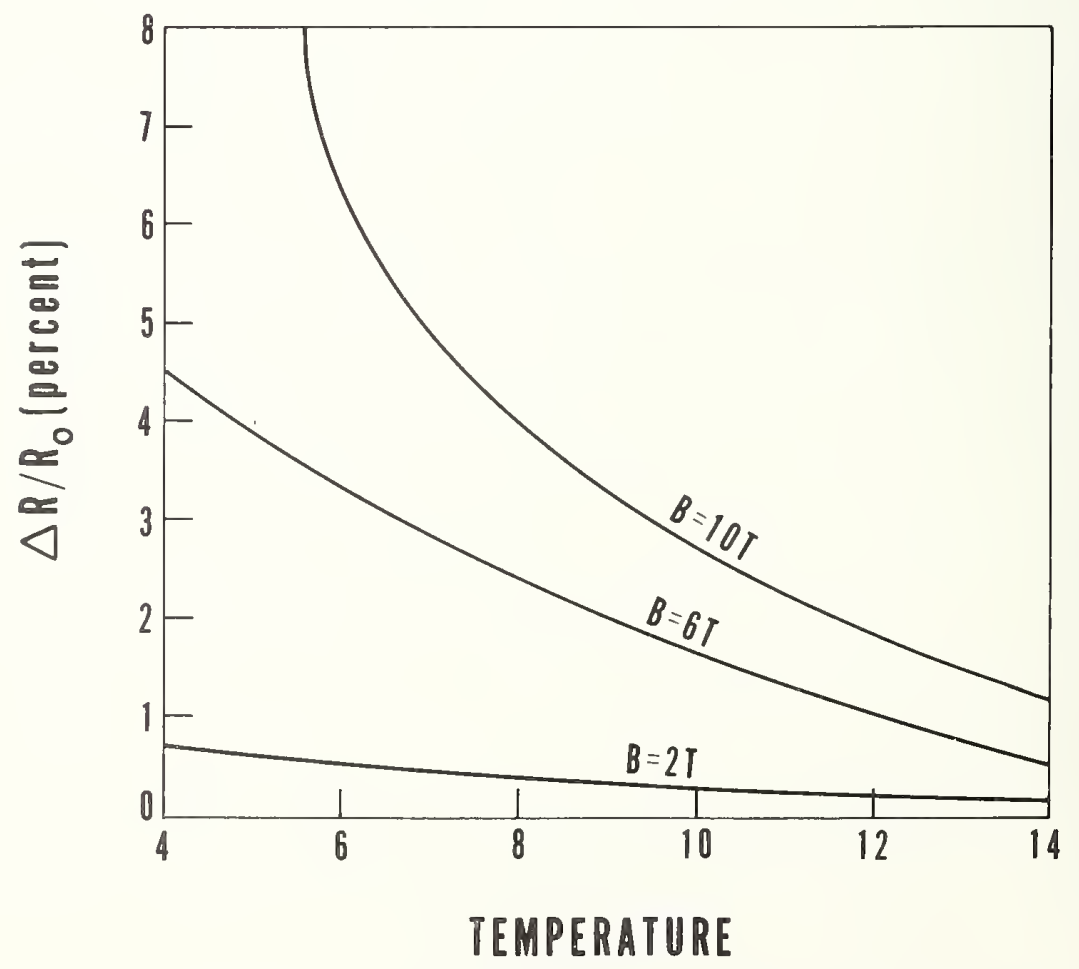

Figure 5.5. Magnetoresistance of carbon resistor thermometers. 


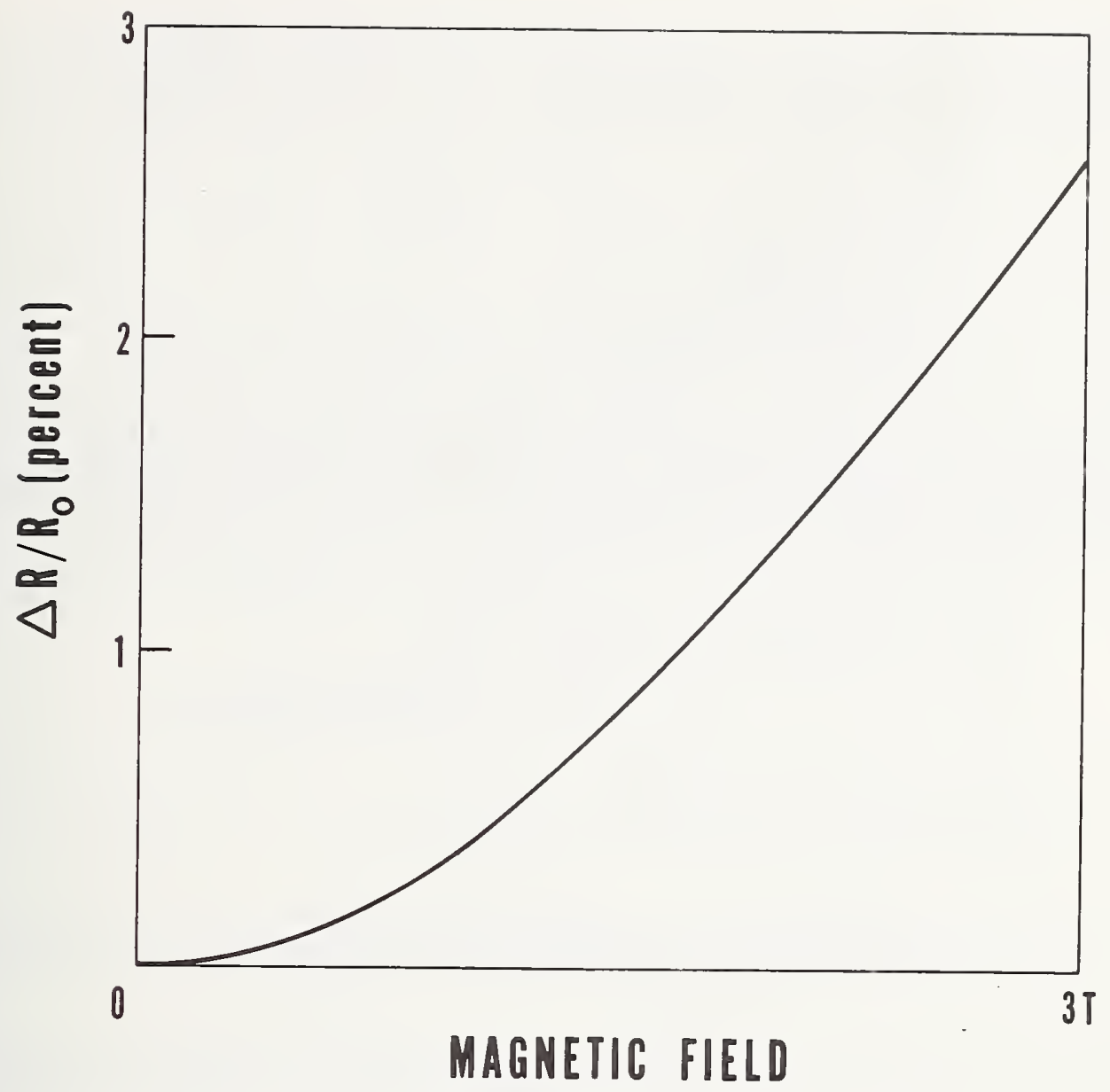

Figure 5.6. Magnetoresistance of carbon-glass thermometers at $4.2 \mathrm{~K}$.

\subsection{Recommended Program}

Two aspects of the thermometry problem need more careful study at this time:

1) Thermometer adaptation, and

2) Instrumental problems associated with long electrical leads at high voltage in high electromagnetic fields.

\subsubsection{Thermometer Adaptations}

Although a number of presently existing thermometers seem potentially acceptable for reliable thermometry in superconducting cables, there remain the problems of encapsulating various thermometers so that they will fit into prototype superconducting cables. There is also the strong possibility that much better thermometer systems (e.g., glass-ceramic capacitors) may be developed at a relatively low cost. At all times the dux? needs for thermometers of two different characteristics must be kept in mind. First, there is a requirement for relatively accurate, thermometers for field and prototype models and later for utility power transmission systems. Second, there is a need for less precise, small, fast response time ( 1 millisecond) thermometers that can be used as diagnostic tools in laboratory research and development. Since the design of transmission lines is not yet determined (in fact, the options for d.c. or a.c. are not even settled), there must be considerable flexibility in ascertaining the criteria for a working thermometry system. 
Thermometer adaptations will require repeated close interaction with the main programs at Brookhaven, Linde, Los Alamos. The project leader will have to systematize the probable requirements for thermometry with respect to:

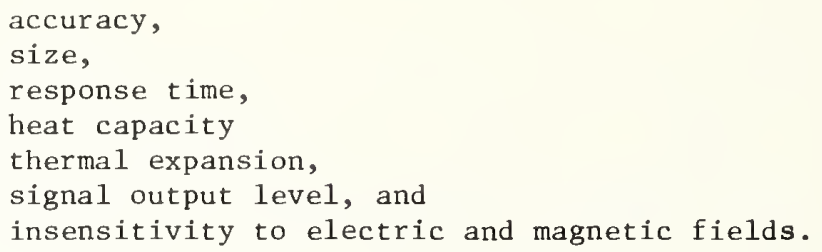

With all those criteria in mind, the physical phenomenon to be probed to determine the temperature will have to be determined. It is almost certain that no single thermometer will fit all of the requirements: At least two different thermometers will be required to cover the extremes in response time and accuracy. At a more practical level the following additional specifications will have to be determined:

Means of attachment of the thermometer to the cable, size and placement of the signal leads, thickness and type of thermometer encapsulation and method of transmitting the signal to a safer environment.

Some preliminary thoughts on the above requirements and specifications can be given:

Accuracy. The diagnostic type thermometers should have an accuracy of about $0.1 \mathrm{~K}$; the semi-permanent ones, about $0.01 \mathrm{~K}$.

Size. The diagnostic thermometers should be flat, 2-5 mm thick, but their length is not so critical, $1-3 \mathrm{~cm}$ long. The larger thermometers may be as large as $1 \mathrm{~cm}$ in diameter, and again their length is not critical.

Response time. This is a critical property; methods of reducing response time usually simultaneously reduce the accuracy. Diagnostic thermometers should have a response time as fast as 1-10 milliseconds; the larger thermometers are slower, say 0.1 to 1 second.

Heat capacity. The property is usually not very adjustable. The diagnostic thermometers will probably have no significant encapsulation. The thicker encapsulation of permanent thermometers leads to higher heat capacities, slower responses, better heat transfer, and more self-heating (if the case is in a varying field).

Thermal expansion. The thermal expansion of the thermometer capsule should match that of the cable part that it is attached to. The material will depend on the cable then, rather than on the type of response time desired, etc. Thermometers attached to aluminum supports may have to have different glues or insulating materials from those interwoven into dielectric layers.

Signal output level and insensitivity to fields. These parameters will have to be determined as the project progresses. They will represent a considerable compromise between those most desirable for instrumentation measurement and the practical requirements of a working system in a high voltage cable.

Attachment. For most systems a metal-filled epoxy can serve as a suitable glue. For thermometers with metallic encapsulation, soft soldering to metallic parts of the cable will probably afford the best response times and thermal equivalence.

Size and placement of signal leads. These will depend on the type of thermometer selected. Both the leads and the thermometers themselves have to be protected so that they will not se ruptured during the field assembly of a complex cable. Very small coaxial cables have been developed for cryogenic usage. These will be especially useful if a frequency signal (rather than voltage) is transmitted from the thermometer to the measuring instrumentation. 
Thermometer encapsulation. Much of the effect caused by the electromagnetic fields can be shielded by encapsulating the thermometers in a metallic reentrant tube. Because of size limitations the shielding can probably only be provided for the larger thermometers that are attached to a free surface in the transmission line. The smaller diagnostic thermometers could probably have little or no shielding. The metallic shield can serve several functions. Both d.c. and a.c. electric fields can be virtually eliminated from the active thermometer region. If a high permeability nickel alloy is used for the shield encapsulator, then much of the d.c. and a.c. magnetic fields can be deflected. The encapsulation can serve three other functions as well: It can serve as a mechanical attachment (permanent or removable) for placing a thermometer on various surfaces. The attachment could be made with epoxies, varnishes, soft-solder, or bolts while the thermometer itself is protected. The capsule can serve as an isothermal body of large heat-conducting surface area so that the thermometer can more closely approximate the temperature of the test body. The same heat conducting surface can serve to help remove any energy generated by self-heating or induced field-dependent heating in the thermometer. And lastly, a properly designed capsule can serve as an electric field shaping conductor that will help to prevent voltage breakdown that could be caused by the sharp edges that are on most thermometers. The crosssection of a proposed encapsulation is shown in figure 5.7. Another possibility is the production of thin film, but well insulated, carbon film thermometers [34].

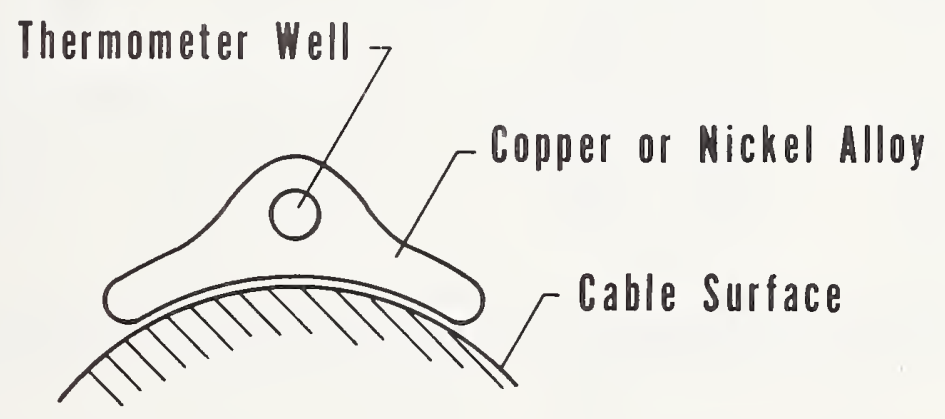

Figure 5.7. Thermometer encapsulation.

New types. One of the most promising of the novel thermometers would be glass-ceramic apacitors inserted in a frequency ratio circuit, a proposal suggested by Lawless (1971) but never put into practice. The most common method of measuring capacitance thermometers is to use a transformer ratio-arm bridge, though phase sensitive detection and frequency ratio methods have also been suggested. The capacitors can be used either in a simple tank circuit (figure 5.8a) or in a more complex frequency ratio circuit.

The latter concept can be illustrated simply in figure 5.8b. A thermally active capacitor, $\mathrm{Ca}$, and a passive one, $\mathrm{C}_{\mathrm{p}}$, are connected by an altérnating switch into a tank circuit with an inductor, L. The two frequency signals are fed out to a measuring circuit, alternate layers in one composite capacitor system. The frequency ratio of the circuit with the passive element to the one with the active element can be expressed as

$$
\mathrm{R}=\frac{\mathrm{f}_{\mathrm{P}}}{\mathrm{f}_{\mathrm{a}}}=\left[\frac{\mathrm{C}_{\mathrm{a}}}{\mathrm{C}_{\mathrm{P}}}\right]^{1 / 2} .
$$

Since $\mathrm{C}_{\mathrm{a}}$ is temperature dependent, so is the ratio $\mathrm{R}$. Since frequency measurements can be made accurately and at relatively low cost, this method has excellent potential. The active element should be a strontium-titanate ceramic glass; the passive one a temperature independent material (for example, military specification designation "NPO"). 


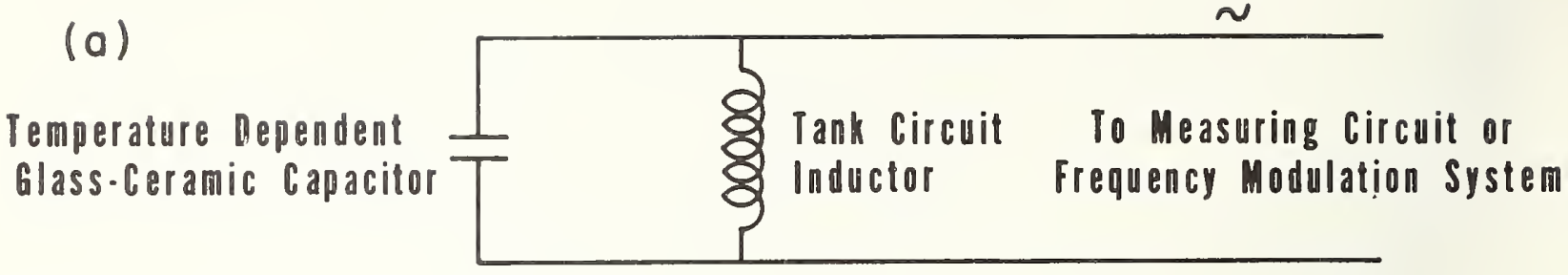

(b)

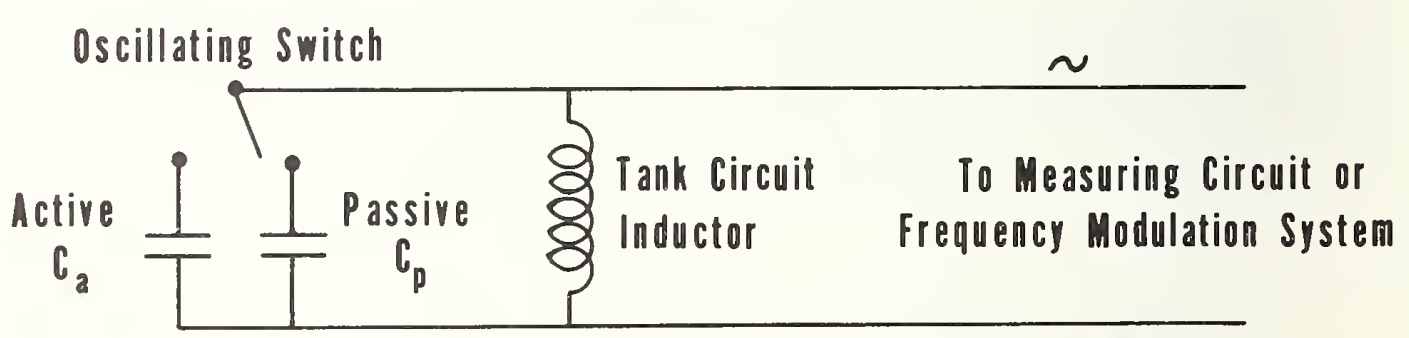

Figure 5.8. Circuit for capacitance thermometers.

Another material that appears to be valuable for further testing is a carbon-impregnated glass. Though this material has a very high temperature sensitivity, it is also more sensitive to magnetic fields than are regular carbon radio resistors.

These various thermometer types as recommended above exhibit no intrinsic difficulties which would make them unuseable in power line applications. However, detailed adaptation and optimization will depend not only the thermometer type and geometrical constraints of the users (not well established at this time), but also on some of the problems of measuring the electrical signals from remotely located sensors in the presence of high electromagnetic noise. We consider these next.

\subsubsection{Signa1 Processing}

There are two effects that can significantly increase the difficulties of accurately transmitting the temperature signals to the outside instrumentation: the high potential (to ground) of the thermometer circuit when it is attached to an active conductor, and the very long spacing, perhaps up to several kilometers, between the transducer and the readout instrument.

The high potential problem is not unique to superconducting power lines, though probably much more instrumentation will be attached to them than to conventional systems, at least in this development stage. All research groups use some type of wire cage to isolate the external high-potential cable components and instruments. If the transducer output is a frequency, transmission of the frequency across this barrier could be accomplished by radio or modulated optical techniques without loss of measurement accuracy. Such techniques are illustrated in figure 5.9. If the transducer output is a voltage, some sort of voltage to frequency conversion may be necessary within the cage (i.e., at high potential) before transmission to ground, thus introducing a measurement uncertainty which we have not evaluated at this time. In either case, some power will be required to run the transducer (i.e., the thermometer measuring current) and delivering this precisely controlled power at the line potential is not a trivial problem, especially if the transducer is in electrical contact with the high voltage cable at some point a kilometer down the line. It is further complicated by requiring the instrumentation to function both with 

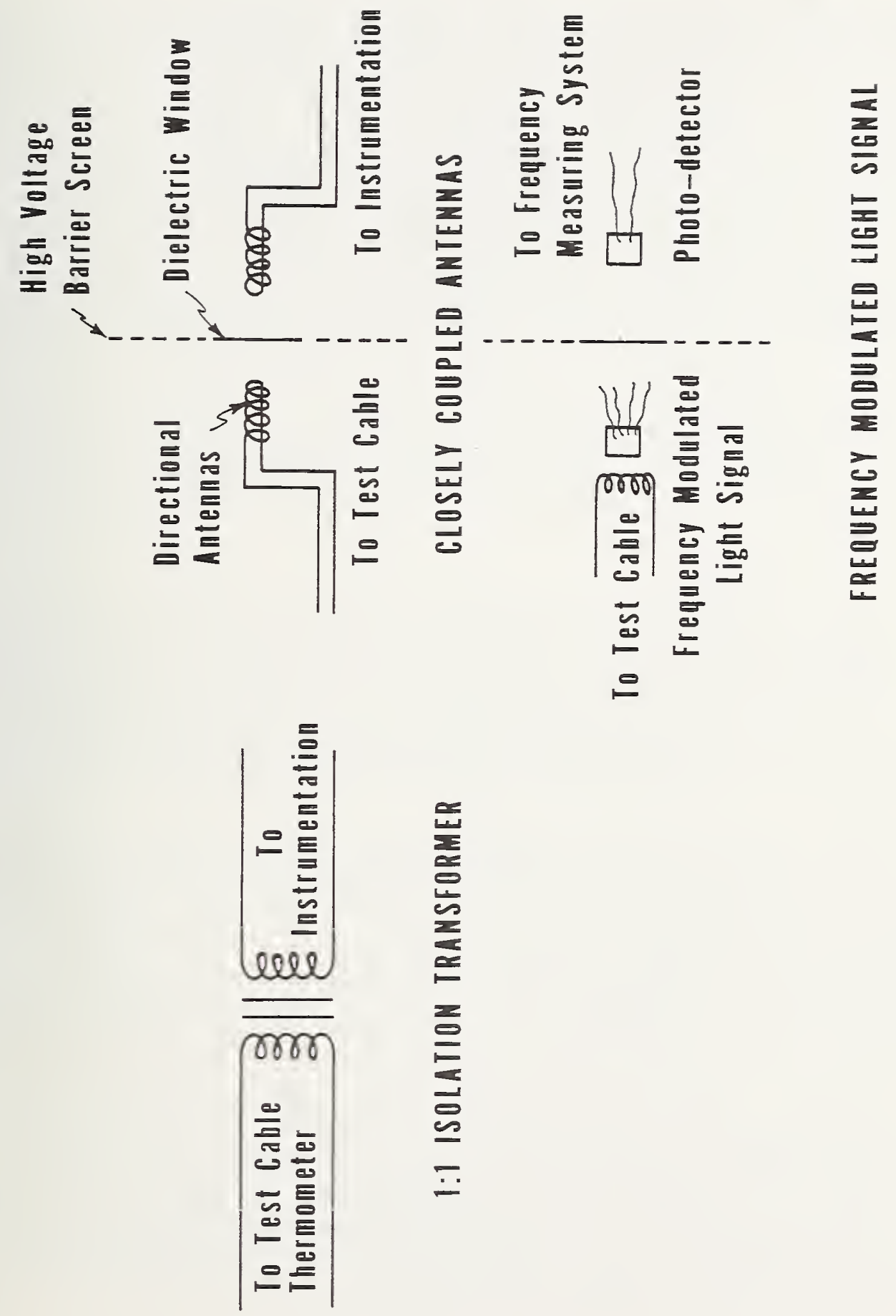
and without high voltage on the power line. The problem seems additionally difficult if d.c. instrumentation is used, and our present inclination is toward a.c. circuitry operating at a kilohertz or more.

A long spacing between the transducer and the readout instruments (in the cage, as above) forces consideration of capacitive and, at $\mathrm{MHz}$ frequencies, inductive effects of the cable on the transducer output signal. It is important that the characteristics of the transmission line not "pull" the output frequency of any thermometer-diode combination which generates a frequency in situ. This has been the problem with some of the past attempts in this direction, though it can be avoided by careful attention to impedance matching. On the other hand, with d.c. instrumentation, the additional voltage drop as the line carries additional current to charge up its own self-capacitance (in response to temperature changes) can be a significant limitation on the frequency response of the measurement system. In addition, the long leads pick up a correspondingly large amount of $60 \mathrm{~Hz}$ noise on the lines. If large enough, and not adequately filtered out, this noise could saturate amplifiers, thus losing the signal. It is necessary to obtain realistic estimates of the noise pickup which may be expected for various practical lead configurations. This again is frequency dependent. At MHz frequencies, coaxial leads would be required, whereas at d.c., superior noise rejection would be obtained by an "inter-eight weave" type of cable.

We have begun to collect and organize relevant information on these two inter-related problem areas, but are unprepared to discourse completely on it at this time. Though discussed here with respect to thermometry, the general concepts apply also to other measurement systems, e.g., pressure transducers. The problems are not new or especially unique, and we are seeking to assimilate existing information rather than to develop new techniques.

\subsection{Conclusion and Future Plans}

For one reason or another, several of the best cryogenic thermometers are not readily suitable for temperature measurements in transmission lines. These unsuitable thermometers include gas volume, vapor pressure, platinum resistance and (marginally) germanium resistance. Carbon radio resistors are suitable for diagnostic tests and may be partially suitable for some kinds of practical systems. The most promising thermometers at present appear to be glass-ceramic capacitors, carbon-impregnated glass, and possibly silicon diodes. The first type is especially suitable because frequency, rather than voltage, signals can be generated by a sensor system.

Superconducting transmission lines generally have only moderate magnetic fields, (of the order of $0.1 \mathrm{~T}$ ). Because of this, the errors in thermometry caused by ambient magnetic fields will be relatively small (for the more suitable thermometers) and those deviations can probably be corrected for rather easily and accurately. The effects of high gradient electric fields can be more serious. However it is probable that they can be made negligible with proper design of thermometer encapsulations. The main problems seem to be a) the adaptation of favorable thermometers to fit specialized instrumentation requirements in a transmission line and b) the difficulty of removing accurate sensor readings from a noisy electromagnetic environment that is at a high electric potential above ground.

It is our considered judgement that the study of high-potential, long lead signal processing, must be carried to a reasonable conclusion before embarking on a specific program of thermometer adaptation and testing as implicity suggested in 5.5.1, above. Having identified thermometer types of principle interest, the detailed form and configuration will depend to some extent on the measurement system with which it will be used, in particular its operating frequency, and its type of output (a voltage amplitude, or a frequency). This system study will be completed within the next reporting period, and should form a basis for subsequent adaptation and testing of appropriate thermometers, in conjunction with the needs of the power line programs. 
1. Belanger, B. C., Paper J-1, Adv. in Cryogenic Engr. Vol. 19 (1974).

2. Belanger, B. C., Cryogenics 15, 88 (1975).

3. Richards, D. B., Edwards, L. R. and Legvold, S. J., J. Applied Phys. 40, 3836 (1969).

4. Sparks, L. L. and Powell, R. L., J. Research NBS 76A, 263 (1972).

5. Sparks, L. L., Powel1, R. I. and Hail, W. J., NBS Nonograph 124 (1972).

6. Powell, R. L., Hall, W. J., et al., NBS Monograph 125 (1974).

7. MacDonald, D. K-C., Thermoelectricity: an introduction to the principles (John Wiley and Sons, Inc. New York, 1962).

8. Schlosser, W. F., Private communication to I. G. Rubin.

9. Berman, R., Kopp, J., Slack, G. A., Walker, L. T., Phys. Letters 27 A, 464 (1968).

10. Berman, R., Temperature, Vol. 4, part 3, 1537 (1972).

11. Sample, H. H., Neuringer, I. J., Rubin, L. G., Rev. Sci. Instr. 45 , 64 (1974).

12. Sample, H. H., Neuringer, L. J., Rubin, L. G., Low Temp. Physics 13, Vol. 4, 601 (1974).

13. Riddle, J. L., Furukawa, G. T., Plumb, H. H., NBS Monograph 126 (1973).

14. Neuringer, I. J. and Shapira, Y., Rev. Sci. Instr. 40, 1314 (1959).

15. Neuringer, L. J., Perlman, A. J., Rubin, L. G. and Shapira, I., Rev. Sci. Instr. 42, 9 (1971).

16. Swartz, J. I., Swartz, D. L., Rev. Sci. Instr., to be pubIished.

17. Unsworth, J., and Rose-Innes, A. C., Cryogenics 6, 239 (1966).

18. Logvinenko, S. P. and Brovkin, Y. N., Instr. Exp. Tekhn. 1, 221 (1968).

19. Barton, L. E., Electronics 35, 38 (1962).

20. Swartz, D. I. and Swartz, J. M., Cryogenic Tech. 5, 250 (1969).

21. Arends, J. and Wright, R. C., Cryogenics 9, 281 (1969).

22. Swartz, J. M. and Craines, J. R., Temperature Vo1. 4, part 2, 1117 (1972).

23. Schlosser, W. F. and Yunnings, R. H., Rev. Sci. Instr. 40, 1359 (1969).

24. Schlosser, W. F. and Munnings, R. H., Temperature Vo1. 4 part 2, 795 (1972).

25. Rubin, L. G., Cryogenics 10, 14 (1970). This includes a review of thermometry through 1969.

26. Neuringer, L. J., Rubin, L. G., Temperature Vol. 4, part 2, 1085 (1972).

27. Lawless, W. N., Rev. Sci. Instr. 43, 1743 (1972).

28. Swartz, J. M., Gaines, J. R. and Rubin, L. G., Rev. Sci. Instr. To be published, 1975.

29. Lawless, W. N., Rev. Sci. Instr. 42, 561 (1971).

30. Lawless, W. N., Radebaugh, R., Soulen, R. J., Rev. Sci. Instr. 42, 567 (1971).

31. Rubin, L. G. and Lawless, W. N., Rev. Sci. Instr. 42, 571 (1971).

32. Cryogenic Data Center, Bibliography on Themometry (1975).

33. Lawless, W. N., Unpublished notes (1971).

34. Collier, R. S., Sparks, I. I. and Strobridge. T. R., unpublished NBS report. 


\section{IMPURITY PROBLEMS IN SUPERCONDUCTING TRANSMISSION LINES}

\subsection{Introduction}

The most promising superconductors for transmission lines require helium refrigeration at or below $10 \mathrm{~K}$. Electrical utilities demand the overall system reliability be high - at least higher than that of present helium refrigerators.

One of the most frequent causes of helium refrigerator failures or shutdown is plugging by impurities carried by the helium [1,2]. The helium inventory of a transmission line probably will be many times that of a large helium refrigerator. This report looks into the potential impurity problem in transmission lines and into ways to minimize it. To gain an "order of magnitude" feel of the possible problem, rough estimates of tolerable impurity levels are presented. Since the calculations assume that impurities collect in one location, the calculations probably overestimate the severity of the problem. As the various transmission line designs become finalized the calculations will need refinement.

There are two ways impurities could cause troubles in superconducting transmission lines. First of all, localized impurity accumulation leading to plugging is a potential problem in all of the proposed transmission lines. Secondly, if helium is the dielectric there is the possibility of impurities affecting the voltage breakdown characteristics of helium. Each of the two topics are considered separately. Then we consider methods of minimizing the problem.

\subsection{Accumulation of Impurities}

Plugging problems have always plagued cryogenic refrigerators. At least four different sources of impurities can be present in any refrigeration system:

1. impurities in the original inventory of helium and/or make-up gas,

2. air leakage into the system,

3. oil from the refrigerator compressors, and

4. off-gassing from the walls in the warm sections of the system.

Commercial Grade A helium usually contains 20 to 50 ppm (by volume) of impurities; these are neon (10 to 15), nitrogen ( 1 to 10 ), water ( 1 to 5 ) and lesser amounts of hydrogen, oxygen, argon and carbon dioxide [3]. Of all of these impurities neon is the most troublesome in helium refrigerators because it is in the highest concentration and has a low boiling point $(27 \mathrm{~K})$. Figure 6.1 shows the diameter of a sphere of impurity (assumed to be solid neon) as a function of initial impurity concentration for an $8 \mathrm{~km}$. (5 mi.) $10 \mathrm{ng}$ line with various flow cross-sections. The results are based on the following assumptions:

1. A11 of the impurity collects at one point.

2. The line contains helium at the density of NBP liquid.

These calculations show that commercially available helium probably requires additional purification if the possibility of plugging is to be minimized. An impurity concentration of less than 1 ppm would be desirable.

Air leaks are a major source of impurities in helium refrigerators. Two of the most likely points of leakage are at the suction of the compressor and in low pressure gas holders. Figure 6.2 shows how fast a sphere of solid air (assumed to be nitrogen) grows as a function of leak rate. Again it is assumed that all of the impurity collects at one point in the system. Based on figure 6.2, it appears that the average leak rate should be kept below $0.01 \mathrm{~g} /$ day $(0.0003 \mathrm{SCF} /$ day); this includes the impurities produced by outgassing. This average leak rate should be obtainable by operating the inlet pressure at above atmospheric pressure and by avoiding the use of low pressure gas holders. Calculations indicate that a low pressure gas holder designed to hold the inventory of a large refrigerator could provide a leak rate in the range of 0.5 to $5 \mathrm{~g} / \mathrm{day}$.

Helium contamination by compressor oil is a common problem; in fact, if screw compressors are used, oil is injected into the helium. Therefore, oil removal is an important step in any cryogenic refrigerator. Figure 6.3 shows the rate of growth of a sphere 


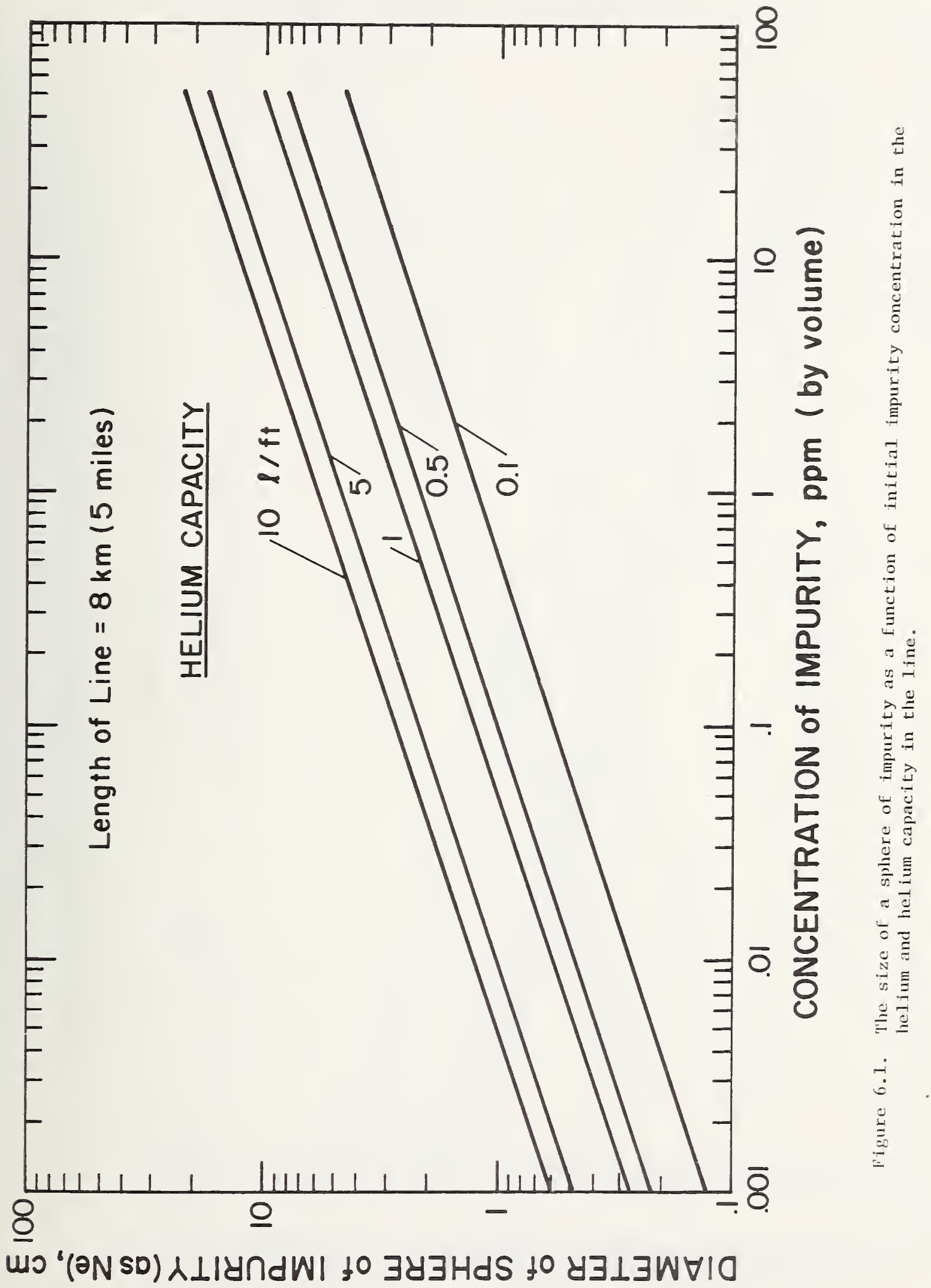




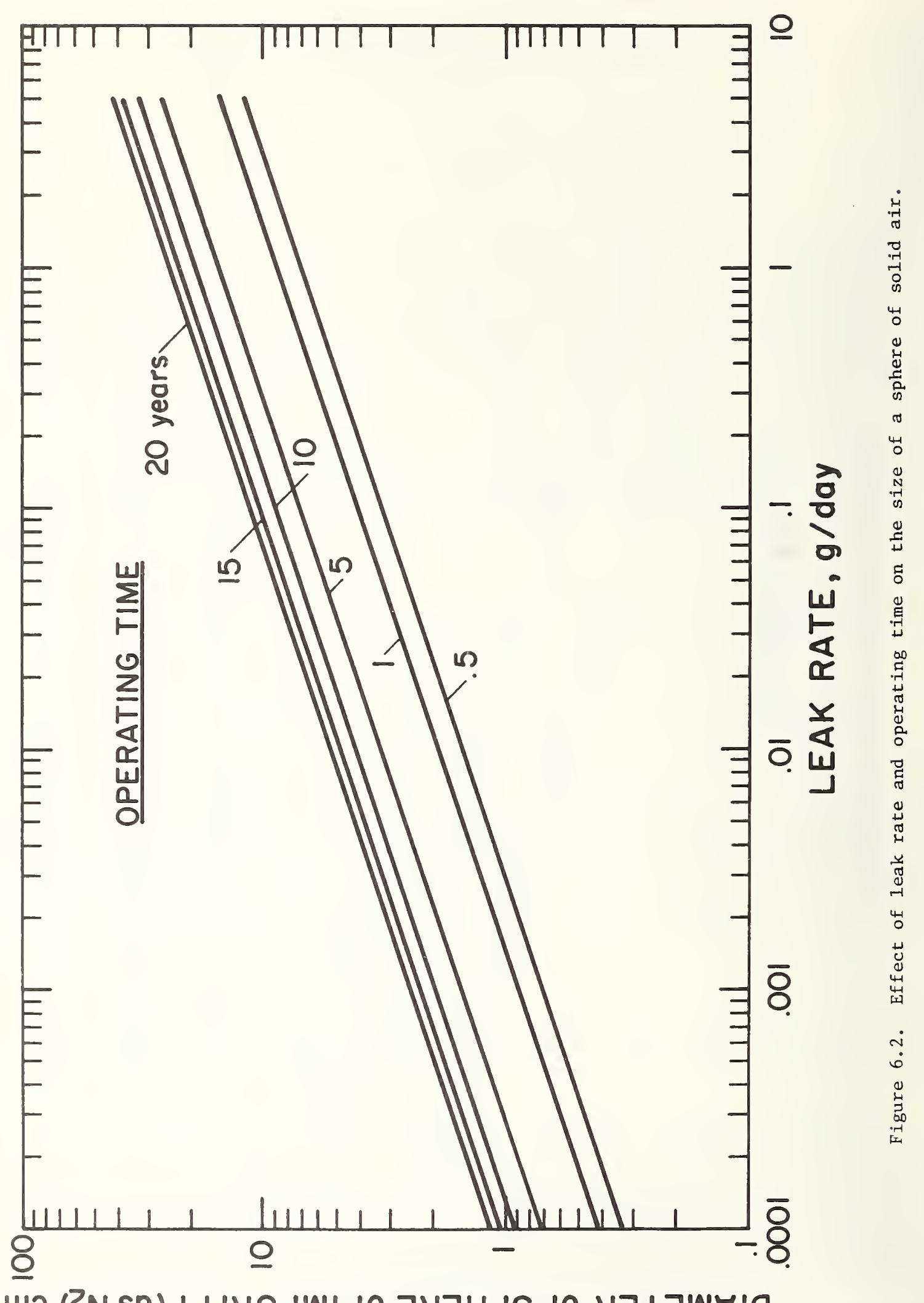



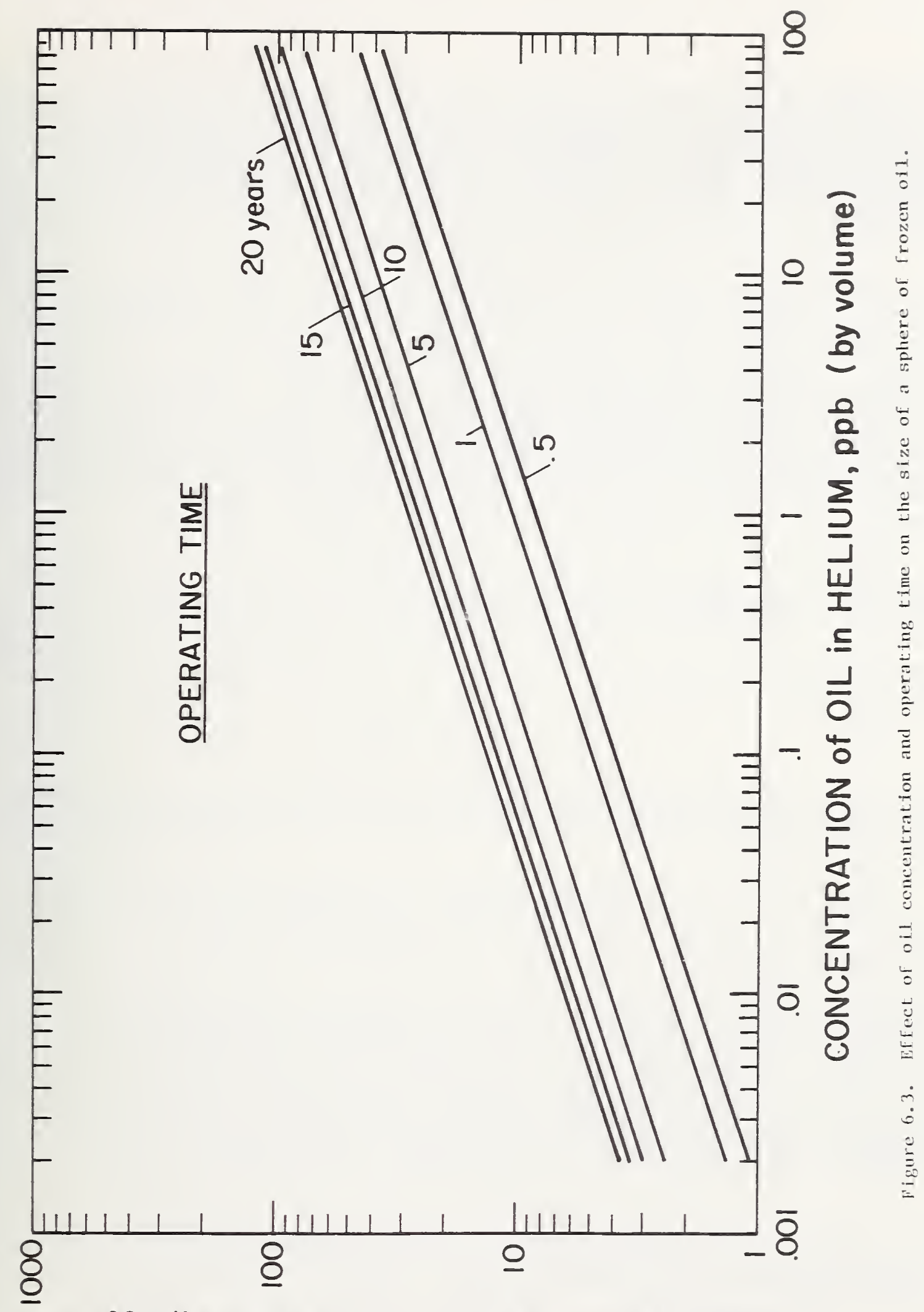

wo ' $\left({ }^{9 \varepsilon} H^{21}\right.$ ○ u SD $) 710$ to $\exists y \exists H d S$ to $y \exists \perp \exists W \forall I 0$ 
of oil (taken to be n-heptadecane) as a function of oil concentration in the helium entering the cold section of the refrigerator. For these calculations, a helium flow rate of $300 \mathrm{~g} / \mathrm{sec}$ is assumed (this corresponds to a $5 \mathrm{Kw}$ refrigerator operating between 8 and 10 K). The sphere diameter is proportional to the cube root of the flow rate. It can be seen that the oil concentration should be less than $0.1 \mathrm{ppb}$ to prevent the possibility of plugging.

\subsection{Effect of Dispersed Impurities on Voltage Breakdown}

If helium is the dielectric, dispersed impurities might lead to a voltage breakdown problem. Meyerhoff [4] found that the addition of solid air or silicon dioxide did not affect the breakdown strength; however, there was a marked reduction in the breakdown strength when copper powder was added to the helium containing the SiO $2^{*}$ In these tests

the impurities were kept in suspension by using a submerged fan. The size of the particles was given ( -325 mesh) but the concentration was not stated. Therefore, it is not clear whether the reduced breakdown strength was due to the presence of a conductor or to the total concentration of particles. However, Meyerhoff [5] feels that the lower breakdown voltage is due to the presence of a conductor instead of the total impurity concentration. Thus it appears that suspended dielectric impurities do not affect the breakdown strength of helium whereas conductors do reduce the breakdown strength.

When helium is the dielectric there is another process which could lead to voltage breakdown; this is dielectrophoresis. Dielectrophoresis is the migration of neutral particles in a nonuniform field [6]. The force on a neutral body is given by

$$
\overrightarrow{\mathrm{F}}=2 \pi \mathrm{a}^{3} \varepsilon_{\mathrm{o}} \phi \nabla\left(\mathrm{E}^{2}\right)
$$

where a is the particle radius, $\varepsilon_{0}$ is the permittivity of the free space, E is the electric field and

$$
\phi=\mathrm{K}_{1}\left(\mathrm{~K}_{2}-\mathrm{K}_{1}\right) /\left(\mathrm{K}_{2}+2 \mathrm{~K}_{1}\right)
$$

where $K_{1}$ and $K_{2}$ are the relative permittivities of the helium and particle, respectively. Since the force depends upon the gradient of the field squared, it is independent of the sign of the field.

Through $\phi$ the force also depends upon the relative permittivity; since helium has a relative permittivity of approximately unity and solids have a value of 4 or more, the particles will be drawn towards the high field region. (Also, because $\mathrm{K}_{2}>1$, the maximum value of $\phi$ will be one).

In transmission lines which use helium as the dielectric the helium flows in the annular space of a coaxial cable. Thus, if dielectrophoresis takes place, impurities will migrate toward the center conductor. If there is a rough spot on the conductor wall or some impurity deposited on the wall, the field gradient near that point will be increased; this, in turn, will cause more impurity to collect at that point. In time this process could cause a stalagtite-type formation to grow. Regardless of the relative breakdown strengths of the helium and impurity, the stalagtite could grow until the distance between the formation and outer conductor was small enough to possibly cause a breakdown in the helium.

One way to prevent stalagtite formation (other than elimirating the impurities) is to have a sufficiently strong convective flow to disrupt the dielectrophoresis effects. The relative strength of the dielectrophoresis forces to that of convective forces can be estimated by comparing the relative velocities. Assuming $\phi$ is one, equation (6.1) for an annular configuration becomes

$$
F=-\frac{4 \pi a^{3} v^{2}}{r^{3} \ln ^{2} k}
$$


where $V$ is the voltage difference between the two conductors, $r$ is the radial position of the particle and $k$ is the ratio of the inner to outer radius of the coaxial cable; the minus sign signifies that the particle moves toward the center conductor. Equation 3 is valid as long as the walls of the conductor are smooth. The force would be greater in the vicinity of a perturbation of the electric field caused by the presence of impurities. Assuming requation 6.3 is valid, the velocity of a particle moving toward the inner conductor can be found by equating the dielectrophoresis force to the viscous force as given by Stokes law. The radial velocity at which these forces are equal is

$$
v_{r}=-\frac{2}{3} \frac{a^{2} v^{2} \varepsilon_{o}}{n r^{3} 1 n^{2} k}
$$

where $\eta$ is the viscosity of helium. (There is also a diffusion force which would oppose the dielectrophoresis force; this effect is probably small enough to be neglected here).

It is expected that the helium flow will be turbulent which complicates the analysis. However, there is a small region near the walls where there is a laminar boundary layer. In this region the axial component of the flow is [7]

$$
v_{z}=\frac{(r-k R) \Delta p R}{2 \mu L}
$$

where $\Delta \mathrm{p} / \mathrm{L}$ is the pressure drop per unit length. (Equation 6.5 is strictly correct only for pipe flow but it is assumed here to apply to annular flow). Combining equations 6.4 and 6.5 and omitting the minus sign gives

$$
\frac{v_{r}}{v_{z}}=\frac{4 \varepsilon_{o} a^{2} v^{2}}{3 S(S+k R)^{3} \ln ^{2} k(k R \Delta p / L)}
$$

where $\mathrm{S}$ is distance from the wall. Putting in the appropriate values of the variables for the Linde line [1] gives

$$
\frac{v_{r}}{v_{Z}}=12800 \frac{a^{2}}{S(24.27 S+1)^{3}} \cong 12800 \mathrm{a}^{2} / \mathrm{s}
$$

where a and $\mathrm{S}$ are in meters. For this case the laminar boundary layer has a thickness of roughly two microns and the buffer zone plus laminar layer thickness is roughly $10 \mathrm{microns}$. Assuming a particle radius of one micron at a distance of two microns from the wall, equation 7 gives a velocity ratio of roughly 0.01 . Since the ratio is so small, convective forces will dominate the motion of the particles, even in the boundary layer. For particles which have a diameter greater than the laminar boundary layer thickness the effect of dielectrophoresis will be dominated completely by the convective forces of the turbulent core. Therefore, it appears that dielectrophoresis will not be important as long as there are no electrical field and fluid flow perturbations. In the regions of surface bumps and/or low flow rates, the effect of dielectrophoresis would be more pronounced; however, it is not obvious whether or not it could cause operational problems.

\subsection{Methods to Minimize the Impurity Problem}

Several options are available which would minimize the impurity problems discussed above.

\subsubsection{Purification of Helium Supp1y Gas}

Considering first the impurities in commercially available helium, either 1iquid filtration or low temperature adsorption could be used for the initial filling of the line, and for the make-up gas [8]. Filtering liquid helium from beneath the liquid-vapor 
interface reduces the total impurity content to around $0.01 \mathrm{ppb}$.

If adsorption is used to remove the neon the adsorption bed must be operated at or below $77 \mathrm{~K}[3]$. If the bed is at $35 \mathrm{~K}$ or lower, it can be regenerated with Grade A helium. However, if higher temperatures are used the bed must be regenerated with a portion of the ultrapure helium; roughly 40 percent of the ultrapure helium is recontaminated by regeneration [8].

Lsing adsorption beds currently requires periodic analysis of the effluent to detect impurity breakthrough. Harrison [9] recently reviewed the commercial instrumentation available for detecting trace impurities in helium. By using only filtration, this periodic gas analysis would not be mandatory.

\subsubsection{Purification of Recirculating Helium}

To adequately remove any air which leaks into the refrigeration system requires at least two adsorption beds - one operating at ambient temperature for water and carbon dioxide removal and one operating at liquid nitrogen temperature for nitrogen, oxygen and argon removal. The bed effluents should be checked several times a day to determine when the bed has become saturated. Extreme care will be required to assure that the samples will not be contaminated by air. However, standard laboratory sampling techniques could be applied here at least at the prototype stage. Field sampling could be more difficult.

Yonitoring the impurity concentration in the adsorption bed would eliminate the necessity for analysis. Only a qualatative indication of when the impurity concentration front reaches a given point in the bed would be required. Physical properties of the adsorbent such as conductance, capacitance or thermal conductivity might be used. However, developmental work will be required to determine which, if any, of the physical properties is the most attractive.

A possible alternative to adsorption beds is to place filters at various points in the refrigerator provided that all of the impurities collect in the filters instead of in critical points in the system.

The practicality of using a filtration system depends upon several factors. If the impurities form particles that are larger than three microns, commercial filters can be used. If the particle dimensions are one micron or less, then filtration would be unpractical. Also, the refrigerator must have a configuration which would be suitable for locating filters in the proper temperature zones. For example, air freezes in the 60 to $55 \mathrm{r}$ range. Thus, it would be desirable to have an expander outlet temperature around 60 K. The outlet gas could then go directly to a filter. In this way the impurities could be trapped before entering a region such as a heat exchanger where the solids could be deposited and cause plugging.

Compressor oil removal from helium requires a demister and adsorption bed (this bed also could be used for water and (:arbon dioxide removal). The problem of oil removal is under study at Brookhaven vationil Laboratory.

\subsubsection{System Configuration}

A system which separates the helium flow in the refrigerator from the flow in the transmission line looks very attractive for maintenance of purity in the high voltage sections of the system. This pumped loop system offers at least two advantages over the single loop system. First of all, if one component fails, the other component may not require shutdown if the downtime is short. Secondly, the pumped loop system would essenLially eliminate the addition of impurities into the helium circulating through the transmission line. Since the helium would remain far below ambient temperatures, out-gassing in the long line would be eliminated. A possible source of impurities would be from the make-11p gas. Because the pumped loop would be isolated from the refrigerator, the make-up rate in the loop should be minimal. 
The major disadvantage of the pumped loop configuration is the additional capital cost for the heat exchanger between the pumped and refrigeration loops and the pump. However, these costs should be small compared to the total cost of the system; the additional cost would probably be worthwile in terms of increased overall reliability. Dean [10] seems to have implied that a pumped loop system would be much less efficient than the direct refrigeration system; however, two other studies [11, 12] have shown that the two systems have comparable efficiencies over much of the operating range of interest.

\subsection{Conclusions and Recommendations}

There is a very real possibility that impurities could adversely affect the operation and reliability of superconducting transmission lines. Simple calculations based on the accumulation of impurities into a single spherical particle show that to minimize the impurity problem:

1. the initial impurity level of the helium put into the system should be less than 1 ppm;

2. the average leak rate of air into the system should be less than $0.01 \mathrm{~g} / \mathrm{day}$;

3. the compressor oil concentration in the helium entering cold

sections should be roughly $0.1 \mathrm{ppb}$ or less.

The above numbers are based on conservative assumptions and should be considered as order of magnitude estimates. As the design of the various transmission lines becomes more finalized, the calculations should be repeated for each specific line geometry.

The impurities can be removed by adsorption and/or filtration. A drawback to using adsorption with present-day technology is that the effluent must be analyzed frequently and with great precision to determine when the bed is saturated. Although there would be no sampling problems when analyzing an ambient temperature bed, there could be problems in sampling the effluent from low temperature beds where air is the impurity and a likely contaminant of the sample.

It should be pointed out that the major objection to analyzing the effluent is not the analyses itself, but in obtaining a reliable sample. Analytical equipment exists that can detect impurities in the ppb range [9]. However, obtaining a sample which contains less than one ppm of contaminants in a field operation will be difficult. Therefore it is recommended that alternates to sampling and analysis be considered. There are at least four options which might be better. Some criteria for selecting which, if any, of the alternates is the best include reliability, reproducibility, sensitivity, simplicity along with stabilicy during temperature and pressure excursions.

The first alternative is to monitor the concentration of impurities on the solid adsorbant. A paper study would be required to determine which of the physico-chemical properties of the solid phase were the most promising as an impurity detector before any experimental work was recommended.

A second alternative would be in situ analvsis of the gas flowing through the bed. There are several physical properties of helium mixtures that might be used as detectors of increased impurity concentration. However, most of these properties are functions of temperature and pressure as well as concentration. Also, the sensitivity to concentration changes might be too low for transmission line applications. Even so, this concept warrants a theoretical feasibility study; experimental work would follow if a promising detection method were found.

Filcration of cold gas is the third alternative to sampling the effluent. Therefore, the feasibility of using filters should be investigated in an experimental program. An important consideration in filtration is the allowable pressure drop through the filters which depends upon the flow rate through the filter and the pore size of the filter. Flow rates can be adjusted by using parallel filters but the pore size depends upon the particle size distribution of the relevant impurities in helium. Thus the size distribution, at least to determine what fraction of particles are less than one micron, should be determined as a Iunction of the contaminant, the leak rate to helium flow ratio, i.e. impurity 
concentration, and the temperature. Commercial filters and gas chromatographs could be used for this experiment.

Lastly, there is the possibility that a combined adsorption bed and filter system could be used. In this system a pressure drop change across the filter could be an indication that the adsorption bed was saturated. Probably the major drawback to filtration, providing the particle sizes were large enough, is the sensitivity of the pressure drop to temperature or pressure fluctuations; such excursions could mask the true pressure drop in the filters. Also, with large temperature excursions, some of the impurities might be "redissolved" in the gas stream and recondensed at some point downstream which could cause plugging.

To improve the overall reliability of a superconducting transmission line the concept of the pumped loop system should be considered. This system would prevent impurities which enter the refrigerator from contaminating the transmission line.

Finally, if helium is to be the dielectric, the possibility of impurities affecting the breakdown strength of helium should be investigated further. Meyerhoff [5] plans to explore the degradation of breakdown strength by impurities in their coaxial cable design sometime in the future. At the time of these tests the possibility of effects due to dielectrophoresis should be considered. 
6.6 References

1. Strobridge, T. R., National Bureau of Standards, Boulder, Colorado, private communication, April 1975.

2. Dean, J. W., Helium refrigeration system reliability for large superconducting systems, Brookhaven National Laboratory Informal Report BNL 19405 (1974).

3. Purer, A., Stroud, L. and Meyer, T. O., Simple technique for the ultrapurification of helium, Advances in Cryogenic Engineering, 10, Plenum Press, New York (1965), p. 398.

4. Meyerhoff, R. W., Development of a rigid AC superconducting power transmission line, Advances in Cryogenic Engineering, 19, Plenum Press, New York (1974), p. 101.

5. Meyerhoff, R. W., Union Carbide Corporation, Linde Division, Tarrytown, N.Y., private communication, April 1975.

6. Poh1, H. A., Nonuniform field effects: dielectrophoresis, electrostatics and its applications, Ed. by A. D. Moore, John Wiley and Sons, New York (1973), p. 336.

7. Bird, R. B., Stewart, W. E. and Lightfoot, E. N., Transport phenomena, John Wiley and Sons, New York (1960), p. 163.

8. Seitz, C. A., Bodine, W. M. and Klingman, C. L., Apparatus and procedure to reduce impurities in helium to less than one part per billion, J. of Chrom. Sci., 9 , (1971) p. 28.

9. Harrison, W. E., Instrumentation for analysis of helium for trace impurities, Brookhaven National Laboratory Informal Report BNL 19570 (January 1975).

10. Dean, J. W., The thermal efficiency of a pumped supercritical refrigeration system, Cryogenics, 14, (1974), p. 387.

11. Cairns, D. N. H., Swift, D. A., Edney, K., and Steel, A. J., Refrigeration and circulation of helium in superconducting power cables, Low Temperature and Electric Power, Proc. Inst. of Refrig., London (1969), p. 155.

12. Daney, D. E., Refrigeration for an $8 \mathrm{~K}$ to $14 \mathrm{~K}$ superconducting transmission line, Nat. Bur. Stand. (U.S.) NBSIR 74-375 (October 1974). 



\section{APPENDIX \\ NEGATIVE DIFFERENTIAL FLOW-RESISTANCE \\ IN SUPERCRITICAL HELIUM* \\ (to be published in Cryogenics)}

V. Arp

Cryogenics Division

Institute for Basic Standards

National Bureau of Standards

Boulder, Colorado 80302

*A contribution of the National Bureau of Standards and not subject to copyright. 


\section{Introduction}

The development of helium cooling systems for large superconducting magnets, power lines, etc., leads to consideration of various helium flow instabilities which can possibly occur in their use. Cooling system instabilities have been widely documented and analyzed for other fluids, at higher temperatures of operation [1, 2, and 3] but until recently there has been comparatively little analysis applied to helium flow instabilities [4,5], though troublesome helium oscillations constitute a well-known lore of cryogenicists. One such instability is the Ledinegg instability, caused by negative differential flow resistance, which sometimes occurs when two-phase or near-critical fluid flows through a heated pipe [4,6]. Depending on circuit parameters external to the heated section, the flow can develop an oscillatory component, or move excursively to a new operating point outside of the region of negative flow resistance. It is the purpose of this paper to discuss the conditions under which a negative flow resistance can occur, but not to discuss the consequent system instabilities which may develop. 


\begin{tabular}{|c|c|c|}
\hline A & $=$ flow cross-section & $\mathrm{m}^{2}$ \\
\hline c & $=$ adiabatic sound velocity & $\mathrm{m} / \mathrm{s}$ \\
\hline D & $\begin{aligned}= & \text { effective diameter of flow channel } \\
& (=4 \times \text { Area } \div \text { perimeter })\end{aligned}$ & $\mathrm{m}$ \\
\hline $\mathrm{f}$ & $=$ Fanning friction factor & -- \\
\hline $\mathrm{H}$ & $=$ enthalpy & $\mathrm{J} / \mathrm{kg}$ \\
\hline $\mathrm{K}_{\mathrm{s}}$ & $=\left.\frac{P}{\rho} \frac{\partial \rho}{\partial P}\right|_{S}=\begin{array}{l}\text { dimensionless isentropic } \\
\text { compressibility }\end{array}$ & -- \\
\hline L & $=$ length of heated channel & $\mathrm{m}$ \\
\hline m & $=$ mass flow rate & $\mathrm{kg} / \mathrm{s}$ \\
\hline M & $=$ Mach number $=u / c$ & -- \\
\hline $\mathrm{P}$ & $=$ pressure & $\mathrm{Pa}$ \\
\hline Q & $=\Lambda \mathrm{L}=$ total heat input & W \\
\hline r & $=$ ratio of specific volumes, $V_{2} / V_{1}$ & -- \\
\hline $\operatorname{Re}$ & $=$ Reynolds number & -- \\
\hline $\mathrm{u}$ & $=$ velocity & $\mathrm{m} / \mathrm{s}$ \\
\hline $\mathrm{v}$ & $=$ specific volume $=1 / \rho$ & $\mathrm{m}^{3} / \mathrm{kg}$ \\
\hline $\mathrm{w}, \mathrm{w}^{\prime}$ & $=$ defined by equations 16 and 17 & - \\
\hline $\mathrm{x}$ & $=$ distance along channel, in direction of flow & $\mathrm{m}$ \\
\hline$\Lambda$ & $=$ heat input/unit channel length & $\mathrm{W} / \mathrm{m}$ \\
\hline$\rho$ & $=$ density $=1 / \mathrm{v}$ & $\mathrm{kg} / \mathrm{m}^{3}$ \\
\hline$\varphi$ & $=\left.\frac{1}{\rho} \frac{\partial P}{\partial U}\right|_{\rho}=\left.\frac{\rho}{T} \frac{\partial T}{\partial \rho}\right|_{s}=$ Gruneisen parameter & -- \\
\hline
\end{tabular}




\section{Background}

The cause of negative differential flow resistance can be understood conceptually with the aid of the helium state diagram, figure 1 , and the schematic flow diagram, figure 2. Consider that dense fluid, of enthalpy $\mathrm{H}_{1}$, enters a heated conduit, warming at approximately constant density until the transposed critical line is crossed, at $\mathrm{H}_{\mathrm{tc}}$, and then at ever decreasing density (approximately like that of an ideal gas) to the outlet enthalpy $\mathrm{H}_{2}$ given by

$$
\mathrm{H}_{2}=\mathrm{H}_{1}+\frac{\mathrm{Q}}{\dot{\mathrm{m}}}
$$

The dense, relatively slow moving fluid at the inlet gives rise to a lower pressure gradient than does the dilute, faster moving vapor near the outlet (at the same mass flow rate). An increase in the mass flow rate causes a decrease in $\mathrm{H}_{2}$ and hence a movement of the point where $\mathrm{H}=\mathrm{H}_{\mathrm{tc}}$ towards the conduit exit. As a consequence, a smaller fraction of the conduit length experiences the relatively high pressure drop associated with low density flow. In some cases the decreased length of high velocity flow more than compensates for the higher pressure gradients in both the low velocity and high velocity sections, resulting in a lower total pressure drop as the flow increases.

The first analysis of this type of unstable flow was made by Ledinegg [6] in 1937. Since then, a number of other studies have been made, the most comprehensive of which are by Zuber [1] in 1968, and Hands [4] in 1974. The major problem with all these studies has been the lack of a satisfactory description of fluid properties in the supercritical range and as a result the analyses lack quantitative accuracy. To quote from Zuber, "It is evident from the preceding results that the success of any analysis concerned with the mechanism of flow oscillations and of heat transfer at supercritical pressures will depend to a great extent upon the ability to describe more accurately the thermodynamic state of a fluid and the transition phenomena which take place at supercritical pressures." 
In this paper we start with equations 1-3 which are thermodynamically exact momentum and energy balances for steady one-dimensional, single-phase fluid flow, in all fluid ranges except right at the critical point [8]. For flow through a horizontal tube of constant cross-section:

$$
\left(1-M^{2}\right) K_{S} \frac{1}{P} \frac{d P}{d x}=-\left(1+\varphi M^{2}\right) \frac{2}{D} M^{2} f-\varphi M^{2} \frac{\Lambda}{\dot{m} c^{2}}
$$

$$
\begin{aligned}
& \left(1-M^{2}\right) \frac{1}{c^{2}} \frac{d H}{d x}=-(1+\varphi) \frac{2}{D} M^{4} E+\left(1-(1+\varphi) M^{2}\right) \frac{\Lambda}{\dot{m} c^{2}} \\
& \left(1-M^{2}\right) \frac{d}{d x}\left(\frac{1}{2} \rho u^{2}\right)=(1+\varphi) \frac{1}{D} \rho u^{2} M^{2} f+\varphi \frac{1}{2} \rho u^{2} \frac{\Lambda}{\dot{m} c^{2}}
\end{aligned}
$$

where

$$
c^{2} \rho K_{s}=P
$$

and

$$
\rho \mathrm{uA}=\dot{\mathrm{m}} \text {. }
$$

In numerical work, described later, these equations have been integrated using a precise helium state equation. However, better insight into the problem is obtained by first considering approximate forms of the integrated results.

\section{Analytical Framework}

The system which we analyze is shown in figure 2. The heated section of tube lies between the planes 1 and 2. Between planes 2 and 3 is placed a flow restriction across which occurs a pressure drop

$$
\Delta P_{2-3}=-k\left(\frac{1}{2} \rho u^{2}\right)_{2}
$$

where $\mathrm{k}$ is an unspecified coefficient, and the minus sign indicates a pressure decrease, consistent with equation (1). A pressure restriction downstream of a heated section is known to enhance the possibility of density-wave flow instabilities [5]; an entrance restriction, which we do not include in the system, will tend to inhibit flow instabilities. 
The total pressure drop from 1 to 3 is then found by adding the integrated pressure drop between planes 1 and 2 to $\Delta \mathrm{P}_{2-3}$, or,

$$
-\Delta P_{1-3}=-\int_{1}^{2} \frac{d P}{d x} d x+k\left[\left(\frac{1}{2} \rho u^{2}\right)_{1}+\int_{1}^{2} \frac{d}{d x}\left(\frac{1}{2} \rho u^{2}\right) d x\right]
$$

where the symbols 1 and 2 in the limits and in the subscript refer to properties which are evaluated at the planes 1 and 2 of figure 2 . It is important to note that these integrations must be performed simultaneously with integration of equation (2) for the enthalpy gradient, so that the two independent parameters $\mathrm{P}$ and $\mathrm{H}$ in the equation of state $\rho(\mathrm{P}, \mathrm{H})$ are determined at every point from 1 to 2 .

We make three basic assumptions:

(a) that the fluid velocity is far below sonic velocity, such that

$$
M^{2} \ll 1
$$

(b) that the heat input per unit length of tube, $\Lambda$, is constant, and

(c) that the enthalpy gradient due to the frictional pressure gradient can be neglected in equation (2), i.e., that

$$
\frac{\Lambda}{\dot{m}} \gg \frac{2}{D} \mathrm{M}^{4} \mathrm{fc}^{2}
$$

In this approximation the enthalpy increases linearly along the tube,

$$
\frac{\Delta \mathrm{H}}{\mathrm{L}}=\frac{\mathrm{dH}}{\mathrm{dx}}=\frac{\Lambda}{\dot{\mathrm{m}}}
$$

and the total pressure drop is given by

$$
\begin{aligned}
-\Delta \mathrm{P}_{1-3} & =\frac{2}{\mathrm{D}} \frac{\dot{\mathrm{m}}^{2} \mathrm{f}}{\mathrm{A}^{2}} \int_{1}^{2} \mathrm{vdx}+\left(1+\frac{\mathrm{k}}{2}\right) \frac{\dot{\mathrm{m}} \Lambda}{\mathrm{A}^{2}} \int_{1}^{2}\left(\frac{\partial \mathrm{v}}{\partial \mathrm{H}}\right)_{\mathrm{P}} \mathrm{dx} \\
& +\mathrm{k} \frac{\dot{\mathrm{m}}^{2} \mathrm{v}_{1}}{2 \mathrm{~A}^{2}}+\frac{\mathrm{k}}{\mathrm{D}} \frac{\dot{\mathrm{m}}^{4} \mathrm{f}}{\mathrm{A}^{4}} \int_{1}^{2} \frac{1+\varphi}{\mathrm{c}^{2}} \mathrm{v}^{3} \mathrm{dx}
\end{aligned}
$$

where we have neglected the variation in friction factor, $f$, with velocity, and used the thermodynamic identity 


$$
\frac{\varphi v}{c^{2}}=\left(\frac{\partial v}{\partial H}\right)
$$

In the previous studies, the above integrals, or their equivalents, have been split up into an incompressible fluid zone plus an ideal gas zone, sometimes with the addition of a third zone characteristic of liquid-vapor mixtures. In this study no division into various zones is made. We assume that the fluid conditions at the inlet (point 1) are specified. Using equation (10), these equations can be rewritten,

$$
\begin{aligned}
-\Delta \mathrm{P}_{1-3} & =\frac{2}{\mathrm{D}} \frac{\dot{\mathrm{m}}^{3} \mathrm{f}}{\Lambda \mathrm{A}^{2}} \int_{\mathrm{H}_{1}}^{\mathrm{H}} \mathrm{v} \mathrm{dH}+\left(1+\frac{\mathrm{k}}{2}\right) \frac{\dot{\mathrm{m}}^{2}}{\mathrm{~A}^{2}} \int_{\mathrm{H}_{1}}^{\mathrm{H}_{2}}\left(\frac{\partial \mathrm{v}}{\partial \mathrm{H}}\right)_{\mathrm{P}} \mathrm{dH} \\
& +\mathrm{k} \frac{\dot{\mathrm{m}}^{2} \mathrm{v}_{1}}{2 \mathrm{~A}}+\frac{\mathrm{k}}{\mathrm{D}} \frac{\dot{\mathrm{m}}^{5} \mathrm{f}}{\Lambda \mathrm{A}^{4}} \int_{\mathrm{H}_{1}}^{\mathrm{H}_{1}} \frac{1+\varphi}{c^{2}} \mathrm{v}^{3} \mathrm{dH}
\end{aligned}
$$

where $\mathrm{H}_{2}=\mathrm{H}_{1}+\mathrm{AI} / \dot{\mathrm{m}}$.

It is important to note that the integrands in eq (13) depend implicitly on $\dot{m}$ through their dependence on $P(x)$. However, if the total pressure drop between 1 and 2 is small compared to the average pressure, this contribution to the dependence of $\Delta \mathrm{P}_{1-3}$ on $\dot{\mathrm{m}}$ will be small compared to that from the factors multiplying the integrals or from the upper limits to the integrals. Point by point within the heated channel, the relative dependencies of the specific volume $v$ on the two independent parameters $\mathrm{P}$ and $\mathrm{H}$ is given by the ratio

$$
\frac{\left(\frac{\partial v}{\partial P}\right)_{H} \frac{d P}{d x}}{\left(\frac{\partial V}{\partial H}\right)_{P} \frac{d H}{d x}} \approx \frac{2}{D} \frac{1+\varphi}{\varphi} M^{2} f c^{2} \frac{d x}{d H}
$$

where we have used (1), (2), (8), (9), (10), and the identity

$$
\frac{P}{\rho}\left(\frac{\partial \rho}{\partial P}\right)_{H}=K_{S}(I+\varphi) .
$$


We now make the assumption that this ratio is much less than one, or

$$
2 \frac{\mathrm{L}}{\mathrm{D}} \frac{1+\varphi}{\varphi} \quad \mathrm{M}^{2} \mathrm{f} \frac{\mathrm{c}^{2}}{\mathrm{H}_{2}-\mathrm{H}_{1}} \ll 1
$$

A more stringent requirement is developed later, at least for the case $k=0$, so that eq. (14) does not limit the validity of the results. Using arguments developed later, one can show that (14) will be satisfied for supercritical helium systems provided $\mathrm{L} / \mathrm{D} \ll(10$ to 100$) / \mathrm{M}^{2}$, and also that the last term in eq (13) will be negligible with respect to the second term. These approximations can be reviewed when the calculation is completed.

Differentiation of equation 13 thus yields

$$
\begin{aligned}
\frac{\partial}{\partial \dot{\mathrm{m}}}\left(-\Delta \mathrm{P}_{1-3}\right) & =\left(3 \int_{\mathrm{H}_{1}}^{\mathrm{H}} \mathrm{v} \mathrm{dH}-\frac{\Lambda \mathrm{L}}{\dot{\mathrm{m}}} \mathrm{v}_{2}\right) \frac{2}{\mathrm{D}} \frac{\dot{\mathrm{m}}^{2} \mathrm{f}}{\mathrm{A}^{2} \Lambda} \\
& +\left(2 \int_{\mathrm{H}_{1}}^{\mathrm{H}}\left(\frac{\partial \mathrm{v}}{\partial \mathrm{H}}\right)_{\mathrm{P}} \mathrm{dH}-\frac{\Lambda \mathrm{L}}{\dot{\mathrm{m}}}\left(\frac{\partial \mathrm{v}}{\partial \mathrm{H}}\right)_{\mathrm{P}, 2}\right)\left(1+\frac{\mathrm{k}}{2}\right) \frac{\dot{\mathrm{m}}}{\mathrm{A}^{2}} \\
& +\frac{k \dot{\mathrm{m}} \mathrm{v}_{1}}{\mathrm{~A}^{2}}
\end{aligned}
$$

where $(\partial v / \partial H)_{p, 2}$ is the derivative evaluated at point 2.

It is convenient to define

$$
\mathrm{w} \equiv \frac{\mathrm{v}_{2}}{\frac{1}{\mathrm{H}_{2}-\mathrm{H}_{1}} \int_{\mathrm{H}_{1}}^{\mathrm{H}_{2}} \mathrm{v} \mathrm{dH}}=\frac{\mathrm{v}_{2}}{\mathrm{v}_{\mathrm{avg}}}
$$

and

$$
w^{\prime} \equiv \frac{\left(\frac{\partial v}{\partial H}\right)_{p, 2}}{\frac{1}{H_{2}-H_{1}} \int_{H_{1}}^{H_{2}}\left(\frac{\partial v}{\partial H}\right)_{p} d H}=\frac{\left(\frac{\partial v}{\partial H}\right)_{p}, 2}{\left(\frac{\partial v}{\partial H}\right)_{p}, a v g}
$$




$$
\begin{aligned}
\frac{\partial}{\partial \dot{\mathrm{m}}}\left(-\Delta \mathrm{P}_{1-3}\right) & =\left(\frac{3}{\mathrm{~W}}-1\right) \frac{2 \mathrm{~L}}{\mathrm{D}} \frac{\dot{\mathrm{m} f} v_{2}}{\mathrm{~A}^{2}} \\
& +\left(\frac{2}{\mathrm{~W}^{\prime}}-1\right)\left(1+\frac{\mathrm{k}}{2}\right) \frac{\Lambda \mathrm{L}}{\mathrm{A}^{2}}\left(\frac{\partial \mathrm{v}}{\partial \mathrm{H}}\right)_{\mathrm{p}, 2} \\
& +\frac{k \dot{m} v_{1}}{\mathrm{~A}^{2}} .
\end{aligned}
$$

In physical terms, $w$ and $w^{\prime}$ are the ratios of the specific volume (or volume derivative) at the exit from the heated section to the average of the same quantity over the length of the heated section. As the volume expansion ratio $\mathrm{v}_{2} / \mathrm{v}_{1}$ increases above 1.0 , so will $\mathrm{w}$ and $\mathrm{w}^{\prime}$ (when $v_{1}$ is to the left of the transposed critical line), but at smaller rates. Only when $w$ reaches 3 or $w^{\prime}$ reaches 2 can any term in equation 18 become negative. To see which occurs first, we must investigate the approximate equation of state in the critical region.

\section{The Near-Critical Equation of State}

A clue to the approximate form of the equation of state in the near-critical region is contained in figure 4, which shows that the quantity $c^{2} / \varphi$ is approximately constant at the transposed critical line, and varies only by a factor of about two within the region of interest for this calculation. This is in noticeable contrast to the specific heat, $C_{p}$, for example, which varies by an order of magnitude in the same region. Thus, as a first, rough, approximation, one can let $c^{2} / \varphi$ be a constant, and integrate equation 12 to obtain

$$
\mathrm{v}=\mathrm{v}_{1} \exp \left(\frac{\varphi}{c^{2}}\left(\mathrm{H}-\mathrm{H}_{1}\right)\right)
$$

as the equation of state. This exponential dependence of $\mathrm{V}$ on $\mathrm{H}$ is seen approximately in the helium data, figure 1. From this figure one can estimate that this equation of state would be quite inadequate 
at expansion ratios $v_{2} / v_{1}$, greater than 3 or 4 . However, using this equation, a short bit of algebra shows that it predicts

$$
\mathrm{w}=\mathrm{w}^{\prime} \frac{\mathrm{r} \ell \mathrm{nr}}{\mathrm{r}-1}
$$

where $r$ is the expansion ratio, $v_{2} / v_{1}$. According to eq. 20, an expansion ratio of 4.9 would be required in order that $w^{\prime}=2$, and $r$ must reach 17 before $w=3$. These numbers, especially the latter one, far exceed the realistic range of the model, but they do make the general point that the condition $w^{\prime}=2$ will be reached more quickly than the condition $w=3$. To explore this further, $w(r)$ was calculated numerically from figure 1, along the 2.5 atmosphere isobar, starting at $\mathrm{H}=40 \mathrm{~J} / \mathrm{mole}(\mathrm{T}=4.2 \mathrm{~K})$. The result is shown in figure 5 , along with a similar curve for $w^{\prime}(r)$, calculated from the helium data of reference 7 , though starting at $4.0 \mathrm{~K}$. Also shown is the prediction of equation 20. The important feature is that both $w^{\prime}$ and $w$ reach a maximum of about 2 to 2.1 and then decrease as exit temperatures continue to rise. The conclusion from both these observations is that the condition $\omega^{\prime}>2$ may be attainable in a single-phase expansion but probably not the condition $w>3$. (This latter could probably be obtained by expansion of a subcooled liquid into the two-phase region.)

At this we have established that the necessary (but not the sufficient) condition for the existence of negative differential flow resistance, with or without an exit restriction, is $\mathbf{w}^{\prime}>2$, or

$$
\left(\frac{\partial \mathrm{v}}{\partial \mathrm{H}}\right)_{\mathrm{p}, 2}>2\left(\frac{\partial \mathrm{v}}{\partial \mathrm{H}}\right)_{\mathrm{p}, \mathrm{avg}}
$$

\section{Two-Phase Fluid}

The calculations to this point have utilized hydrodynamic and thermodynamic equations derived for single-phase fluid flow. However, 
to the extent that the flow of a liquid-vapor mixture can be described in terms of the flow of a homogeneous fluid with a well defined stateequation $\mathrm{V}(\mathrm{P}, \mathrm{H})$, the results above can as well be applied to two-phase flow. The previous studies of the Ledinegg instability have all implicitly been based on this assumption. In this respect, the quantity $c^{2} / \varphi$ as used throughout the paper in fact refers to the thermodynamic derivative defined in equation 12, rather than a measured sound velocity, which may be anomalously low in a turbulent liquid-vapor medium.

Starting in the subcooled liquid region of figure 1 , as the fluid enthalpy increases, an isobar experiences a sharp increase in slope as the two-phase region is entered, and a second, but smaller, increase in slope upon entering the vapor phase. Since the isobar is nearly flat in the subcooled liquid region, equation (21) is easily satisfied by isobaric expansion from subcooled liquid into the two-phase region, provided that the pressure is not too close to the critical pressure. This conclusion is quantitatively consistent with the work of Hands [4].

\section{Numerical Estimate of Necessary Conditions}

Values of $\mathrm{v}, \mathrm{H}$, and $\mathrm{v} \partial \mathrm{H} / \partial \mathrm{v}$ are tabulated for helium as a function of pressure and temperature in reference [7]. Simple numerical work with this data leads to figure 5. The inlet pressure and temperature must be within the shaded region to the left of the liquid-vapor line in order to find any isobaric expansion process in which eq. 21 is satisfied; when it is satisfied the final temperature will always be in the two-phase region or very close to the transposed critical line. The lower portion of that shaded region, at subcritical pressures, is predictable from the previous work of Hands [4], while the upper limit, at near-critical and supercritical pressures, is provided by this study. For non-isobaric expansions, the upper limit of figure 5 should be a little higher, e.g., for expansion from 3.0 to 2.4 atmospheres, $w^{\prime}=2$ can be reached at input temperatures as high as $3.4 \mathrm{~K}$. Considering also our neglect of the friction factor variation with temperature, the upper limit in figure 5 should be regarded as an approximate limit to input conditions which could lead to negative differential flow resistance. 


\section{Estimate of Sufficient Conditions for Single-phase Fluid}

Assume, with reference to figure 4, that it may be possible for $w^{\prime}$ to reach a little above 2.0 at the end of the heated section. Because of the factor $\left(1-2 / w^{\prime}\right)$, numerical results are very sensitive to small changes in $w^{\prime}$, and must be discussed with $w^{\prime}$ as an adjustable parameter. On the other hand, for this approximate treatment, let $(3 / w-1)=1$, suggested by figure 4. Then the sufficient condition for a negative differential flow resistance, within our approximations, is

$$
\left(1-\frac{2}{w^{\prime}}\right)\left(1+\frac{k}{2}\right) \frac{\Lambda L}{\dot{m}}\left(\frac{\partial v}{\partial H}\right)_{p, 2} \geq \frac{2 \mathrm{Lfv}_{2}}{\mathrm{D}}+\mathrm{kv}_{1}
$$

or, in more convenient form

$$
\left(1-\frac{2}{w^{\prime}}\right)\left(1+\frac{k}{2}\right) \frac{\Lambda L}{\dot{m}} v_{2} \gtrsim \frac{2 L f c^{2} v_{2}}{D \varphi}+\frac{k c^{2} v_{1}}{\varphi},
$$

where $c^{2} / \varphi$ is evaluated at station 2 .

In the absence of an exit flow restriction $(k=0)$, the enthalpy gradient required for negative differential flow resistance is

$$
\frac{\Delta \mathrm{H}}{\mathrm{L}}=\frac{\Lambda}{\dot{\mathrm{m}}} \simeq \mathrm{C}_{\mathrm{p}} \frac{\mathrm{dT}}{\mathrm{dx}}>\frac{2 \mathrm{f}}{\mathrm{D}} \frac{\mathrm{c}^{2}}{\varphi} \frac{1}{\left(1-2 \mathrm{w}^{\prime}\right)}
$$

For expansions satisfying equation (21), starting in the shaded region of figure 5 and ending near the transposed critical line, one finds that $\Delta H$ will be about $2 \times 10^{4} \mathrm{~J} / \mathrm{kg}$; then, with $c^{2} / \varphi \approx 2 \times 10^{4} \mathrm{~m}^{2} / \mathrm{s}^{2}$ and $\mathrm{f} \approx 0.005$, equation $(26)$ becomes

$$
\frac{\mathrm{L}}{\mathrm{D}}<100\left(1-\frac{2}{\mathrm{~W}^{\prime}}\right)
$$

or $\mathrm{L} / \mathrm{D} \lesssim 10$ if $\mathrm{w}^{\prime}=2.2$. Very short channels, with correspondingly high temperature gradients in them, will be required for the occurrence of negative differential flow resistance in supercritical helium systems, at 
least in the absence of exit flow restrictions. This is in qualitative agreement with a prediction of Hands [4], based upon a two-zone description of fluid properties. It should be noted at this point that equation 27 is a much more stringent restriction on L/D than is equation 14 .

Physically, equation 26 means that the pressure gradient due to acceleration of the heated fluid must be relatively large, at least $2 /\left(1-2 / W^{\prime}\right)$ times the frictional pressure gradient. The acceleration pressure drop can be estimated from eq. 1, within our approximations, as

$$
\left(\frac{\Delta P}{P}\right) \approx\left(\frac{M^{2}}{K_{S}}\right) \operatorname{avg} \frac{\varphi \Delta H}{c^{2}} \text {. }
$$

With $\varphi \Delta \mathrm{H} / \mathrm{c}^{2} \lesssim 1, \mathrm{~K}_{\mathrm{S}} \approx 0.1$, and $\mathrm{M}^{2}<1$ (probably $<10^{-2}$ to $10^{-3}$ ), $\Delta \mathrm{P} / \mathrm{P}$ will be very small, and the expansion will be quasi-isobaric.

Exit restrictions, within the context of this paper, could include orifices, venturis, valves, or just a section of unheated line where frictional pressure drop occurs. It is impossible in this sense to give a "typical" value of $k$, but values as high as $10^{2}$ to $10^{3}$ could be reached with highly constricted orifices.

The exit restriction dominates the requirement when both $k \gg 2$ (from the left-hand side of equation 25) and

$$
\mathrm{k} \gg \frac{\mathrm{v}_{2}}{\mathrm{v}_{1}} \frac{\mathrm{EL}}{\mathrm{D}}
$$

(from the right-hand side). Under these conditions, negative differential flow resistance between 1 and 3 should occur when

$$
\left(1-\frac{2}{w^{\prime}}\right) \Delta H\left(\frac{\varphi}{c^{2}}\right)_{2} \frac{\mathrm{V}_{2}}{\mathrm{v}_{1}}>2
$$

The factors on the left-hand side of this equation are inter-related by the equation of state. Assuming the exponential state equation (19), one finds that this condition is equivalent to $w=w^{\prime}>2.3$. This is not a reliable numerical result, because of the innaccurate state equation 
used to obtain it, but it does suggest that, with a sizeable exit restriction in place, a much more stringent condition on the fluid properties is required to reach the sufficient condition, eq. 28, than to reach the necessary condition, eq. 19. At the same time, the additional requirements of high temperature gradients and short lines, as for $k=0$, do not appear.

Simple numerical work with the helium data [7] shows that no realistic expansion process can be found in supercritical helium (at least starting above the $\lambda$ point) which satisfies equation 28 . Starting an isobaric expansion at $2.5 \mathrm{~K}$, the maximum numerical value one can find for the left-hand side of (28) is about 0.7 at 2 atmospheres pressure; for non-isobaric expansions the number is a little higher, but not greatly so. Thus, this theory predicts that, with a large exit restriction in place, negative flow resistance between planes 1 and 3 will never be found in supercritical helium systems. This is an unexpected result, in that the existence of the exit flow restriction has the effect of damping out this type of possible instability, in contrast to its destabilizing effect on dynamic instabilities [5].

\section{Numerical Integration}

To explore the problem without invoking the various approximations used in this analysis, equations (3) and (4) have been numerically integrated in conjunction with a precise helium state equation of the form $\mathrm{v}(\mathrm{P}, \mathrm{H})$ [8]. The state equation does not correctly describe twophase fluid properties, so that integrations were performed only in the single phase region, for pressures $\geq 2.5$ atm . During the integration, the friction factor $\mathrm{f}$ is calculated from the local value of the Reynolds number by [10]

$$
\mathrm{f}=0.0014+0.125 \mathrm{Re}^{-0.32}
$$

For each integration the inlet pressure and temperature, the mass flow rate, the channel length and diameter, and heat input must be specified. 
A number of different exit coefficients can be used for each integration, since they merely add to integrated $\Delta P_{1-2}$.

The numerical integrations are in substantial agreement with the analysis above. In the absence of a flow restriction, we find that, for single phase flow,

(1) The maximum value of $w^{\prime}$ which can be found for supercritical helium channels is about $\simeq 2.2$, starting at temperatures of about $2.5 \mathrm{~K}$ and pressures of 2 to 2.5 atmospheres, though possibly higher values could be found by expansions ending very near the critical point where our equation of state is not accurate.

(2) Negative differential flow resistance can be found with input conditions of $2.5 \mathrm{~K}$ and 2.5 atmospheres, and very high heat inputs to short tubes, such that $w^{\prime}>2$ at the outlet. This is illustrated by the lower curves in figure 6. As pointed out by Dr. Hands [11], the positions of the maxima and minima in these curves are quite sensitive to small changes in the assumed equation of state of the fluid, and of the assumed friction factor.

(3) The effect disappears for longer tubes, even though all other conditions are satisfied. This is illustrated by the upper curves in Iigure 6 .

(4) With other parameters held constant the effect becomes marginal when the input temperature is raised to $3.5 \mathrm{~K}$ (curve B, figure 7) and is gone at an input temperature of $4.5 \mathrm{~K}$ (curve C); similarly the effect becomes marginal at an input pressure of 3.0 atmospheres (curve D), and is gone at an input pressure of 4.0 atmospheres (curve E). Curve $A$ in this figure is taken from figure 6.

With a large exit restriction in place, negative flow resistance between points 1 and 3 of figure 2 is not found under any condition of the very many that have been tried. This is illustrated in figure 8 , which shows the disappearance of negative flow resistance with increasing values of $k$, starting with fluid conditions under which it is a near maximum for helium systems. 


\section{Conclusion}

We conclude that negative differential flow resistance, and the consequent tendency toward a Ledinegg instability, will not occur in single-phase helium cooling systems except under very restricted conditions of low input temperature, near-critical pressure, very high heat input, short channel lengths, and the absence of a large exit restriction pressure drop.

\section{Acknowledgement}

Michael Jones has contributed to many useful discussions of this topic. Brian Hands has stimulated the work through his publications and lively correspondence. The work was supported by Brookhaven National Laboratory and the ERDA. 


\section{References}

1. N. Zuber, "An analysis of thermally induced flow oscillations in the near-critical and super-critical thermodynamic region", NASA report NAS8-11422 (1966).

2. J. A. Boure, A. E. Bergles, and L. S. Tong, "Review of two-phase flow instability", ASME paper 71-HT-42 (1971).

3. J. C. Friedly and V. S. Krishnan, "Prediction of non-linear flow oscillations in boiling channels", AIChE paper 14 presented at the ASME-AIChE Heat Transfer Conference, Tulsa, Okla (1971).

4. B. A. Hands, "A re-examination of the Ledinegg instability criterion and its application to two-phase helium systems", presented at Symposium on Multi-phase flow systems, Glasgow, Scotland 2-4 April (1974); also, "Pressure drop instabilities in cryogenic fluids," Adv. Cryo. Eng. 20, 355 (1975).

5. M. C. Jones and R. G. Peterson, "A study of flow stability in helium cooling systems", ASME paper 74-WA/HT-24 (1974).

6. M. Ledinegg, Die Warme 61, 891 (1938).

7. R. D. McCarty, "Thermophysical properties of helium-4 from 2 to $1500 \mathrm{~K}$ with pressures to 1000 atmospheres", NBS Technical Note 631 (1972).

8. V. Arp, "Thermodynamics of single-phase, one-dimensional fluid flow", Cryogenics 15, 285 (1975).

9. V. Arp, "New forms of state-equations for helium", Cryogenics 14, 593 (1974).

10. E. C. Koo, ScD thesis, MIT (1932); result quoted in W. H. McAdams, Heat Transmission, third edition, p. 155, McGraw Hi11, N.Y. (1954).

11. B. A. Hands (private correspondence). 


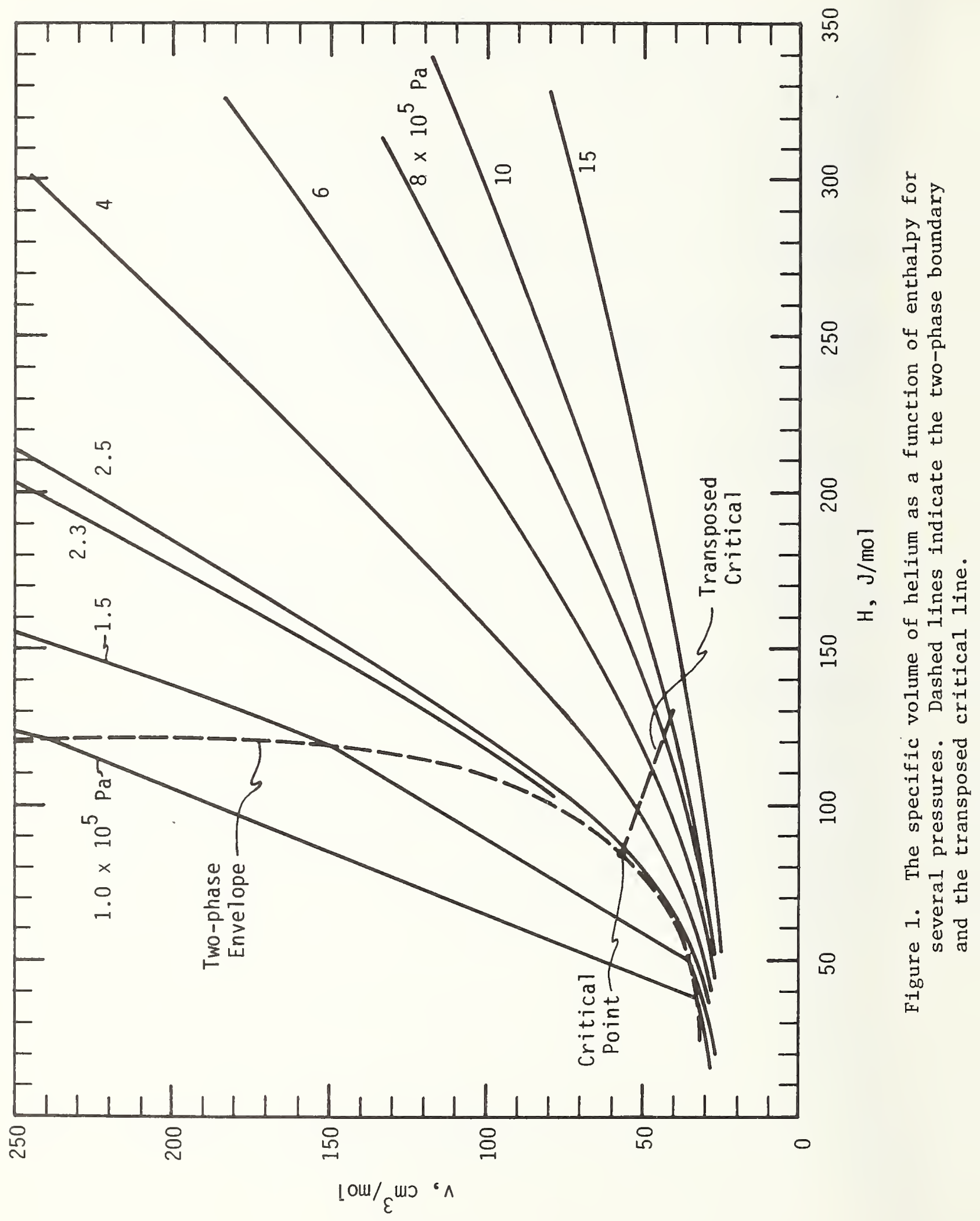




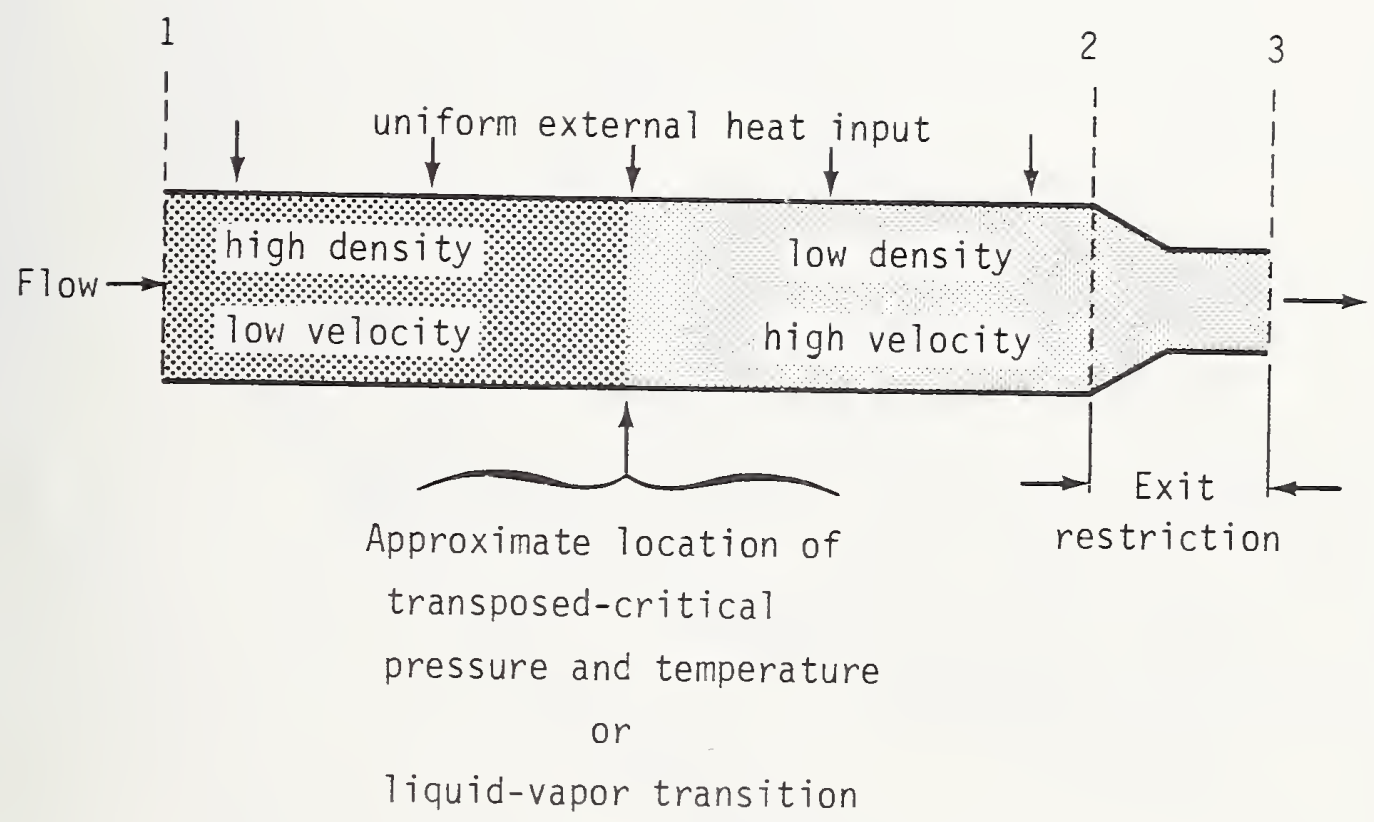

Figure 2. Schematic diagram of fluid flow through a heated channel, defining the planes 1,2 , and 3 respectively before and after the heated section, and after the exit restriction. 


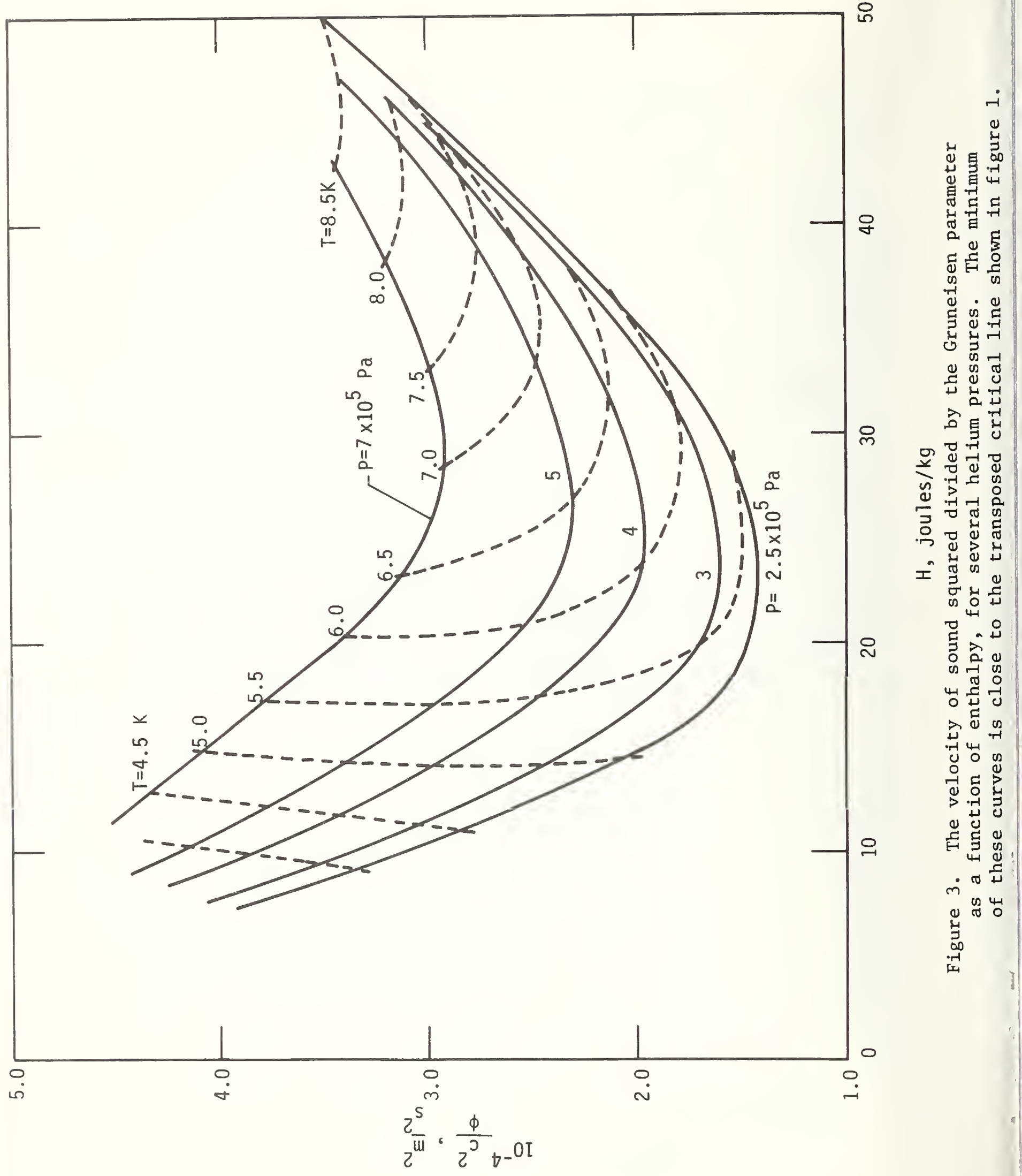




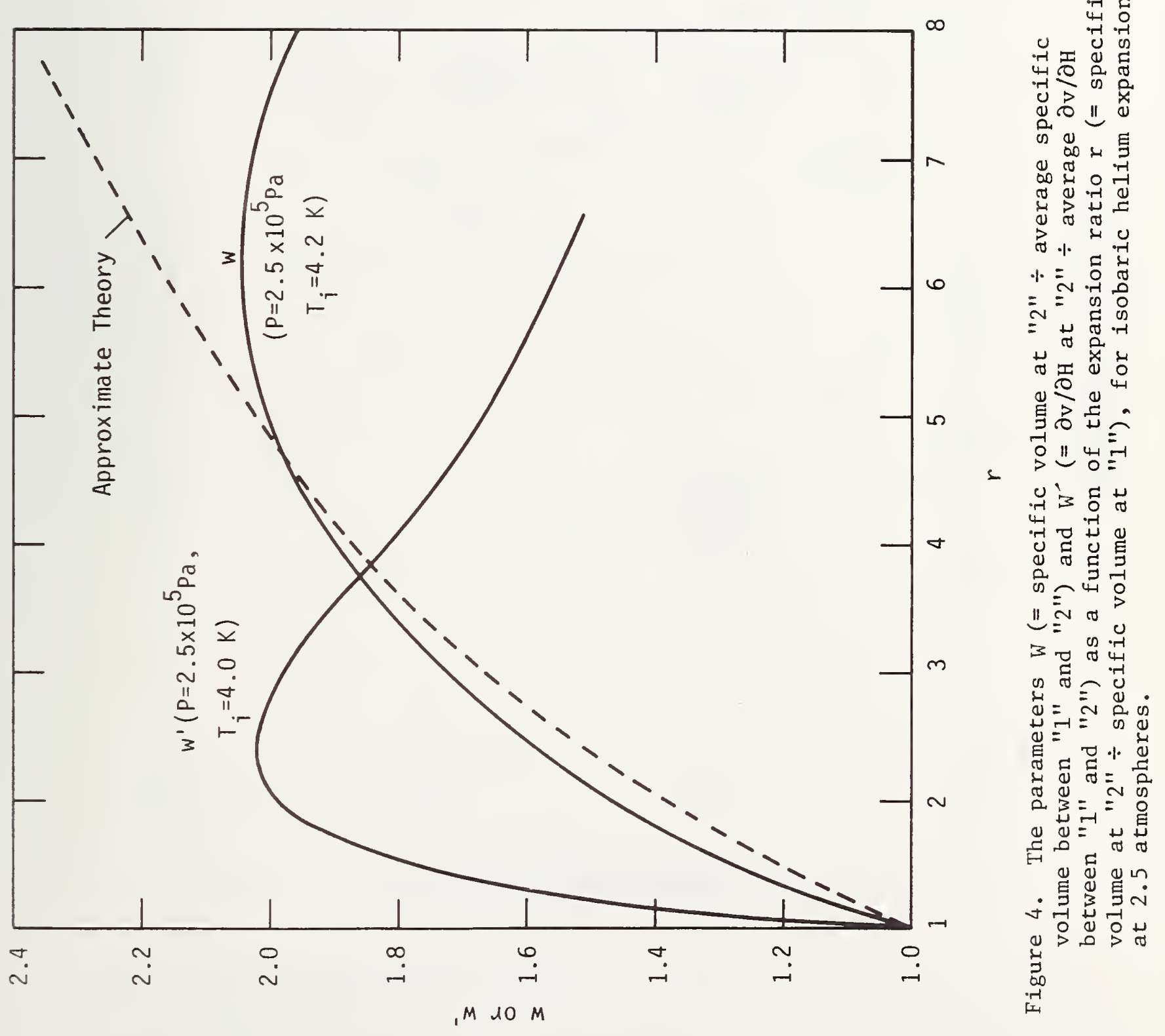




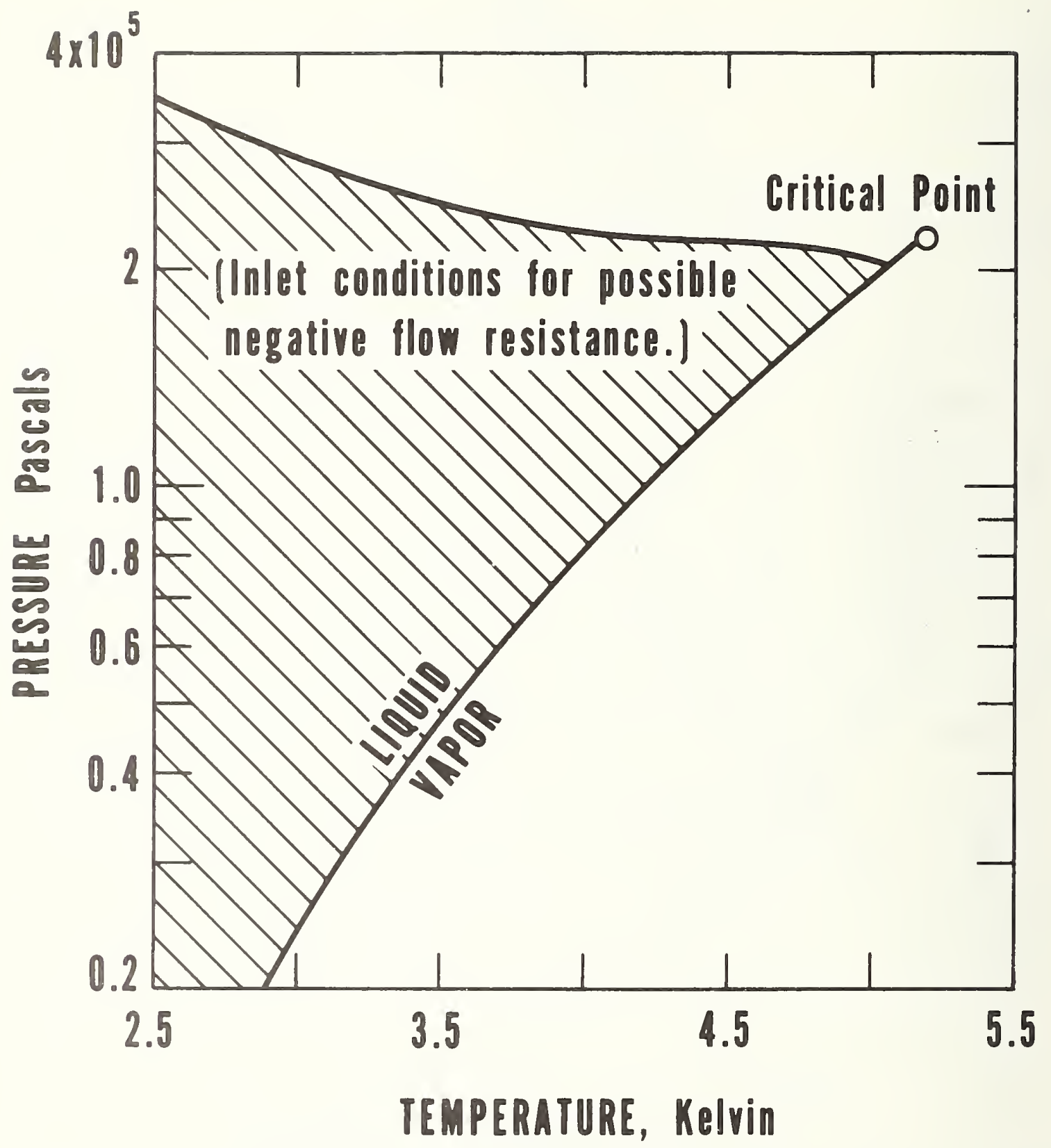

Figure 5. Helium phase diagram. All isobaric expansions leading to $w^{\prime}>2$ (defined by eq. 17) originate within the cross-hatched region on the left: 


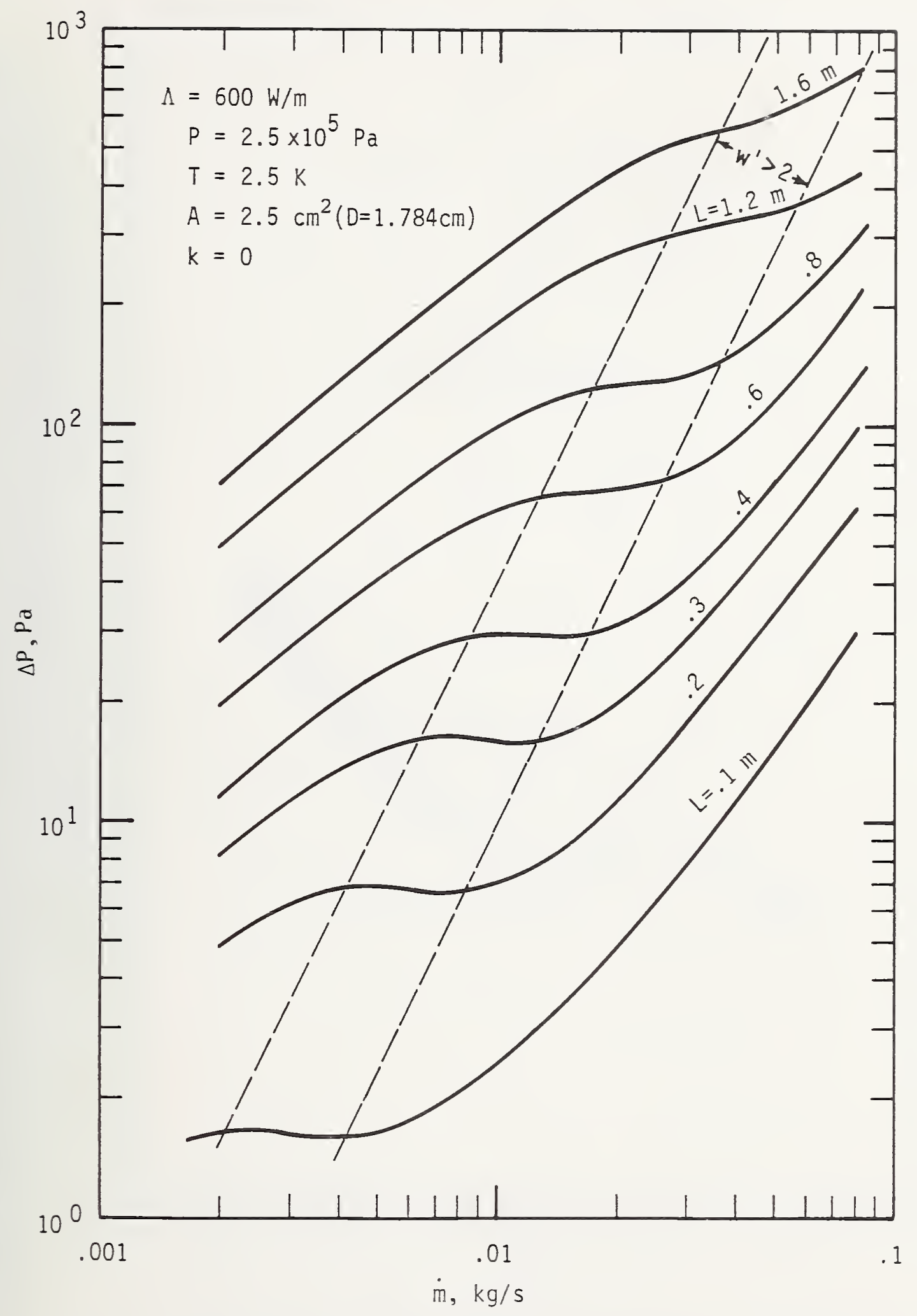

Figure 6. Calculated pressure drop as a function of mass flow rate, for several channel lengths. Between the dashed lines eq. (21) is satisfied $\left(w^{\prime}>2\right)$. Negative differential flow resistance disappears at L/D $\gtrsim 50$ in this example. 


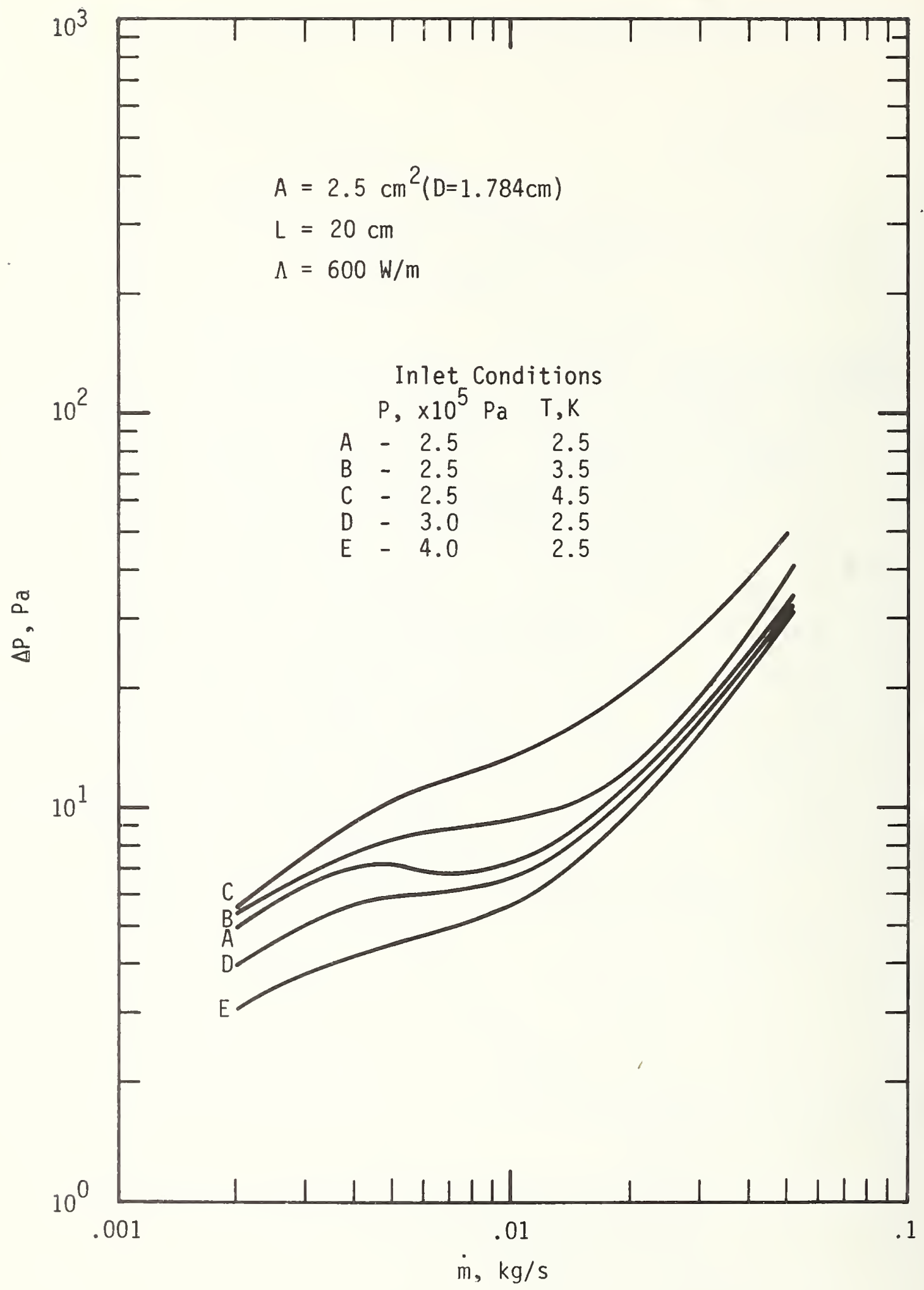

Figure 7. Calculated pressure drop as a function of mass flow rate for several fluid conditions at the inlet (plane "1" of figure 1). Curve $A$ is the $L=0.2$ meter curve from figure 6 . 


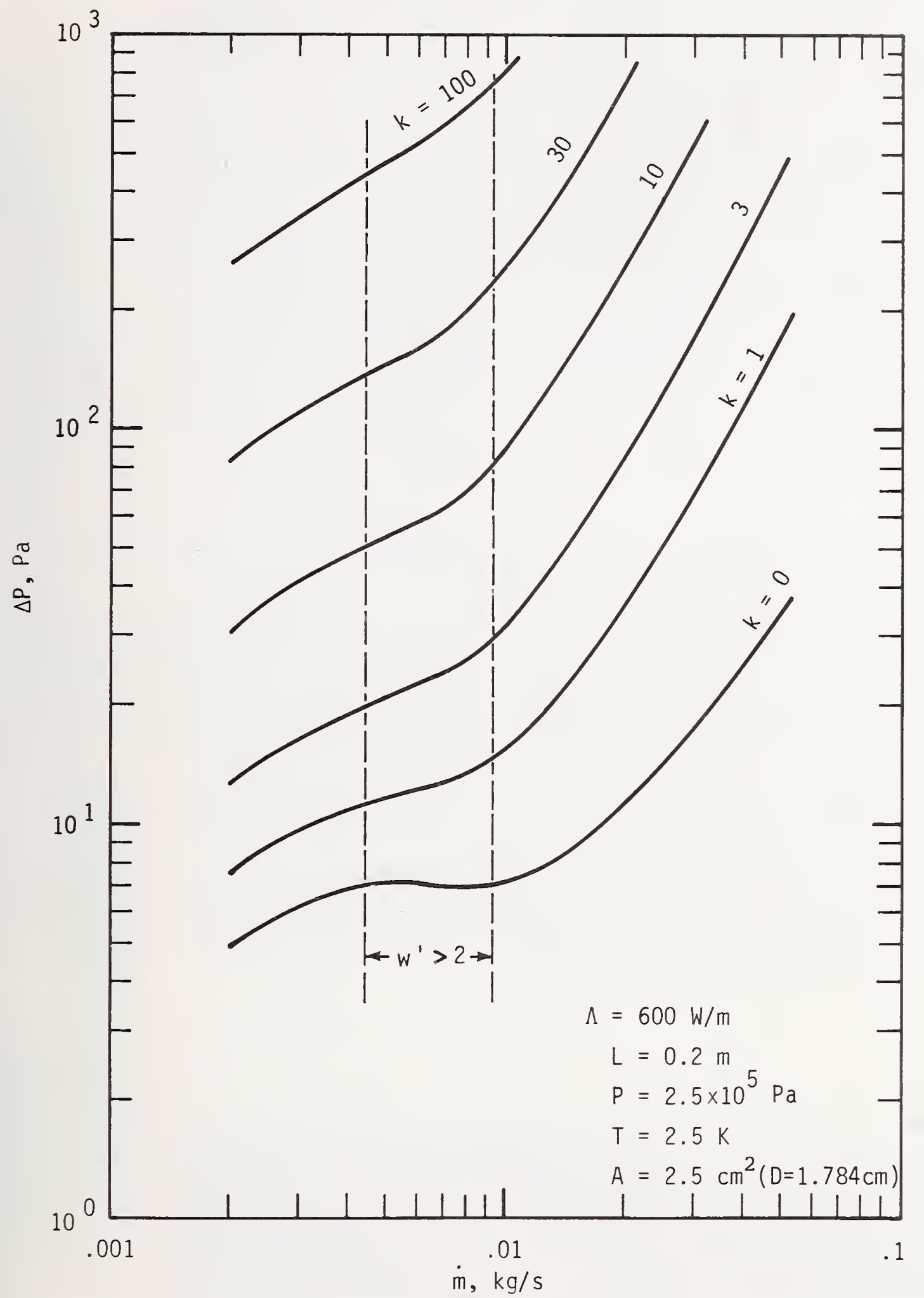

Figure 8. Calculated pressure drop as a function of mass flow rate for several different exit coefficients $k$ (defined by equation 6 ). The $\mathrm{k}=0$ curve is the $\mathrm{L}=0.2 \mathrm{~m}$ curve from figure 6 . Between the dashed lines eq. (21) is satisfied $\left(\mathrm{w}^{\prime}>2\right)$ ). Negative differential flow resistance disappears as the exit restriction ( $k$ ) increases. 
NBS-114A (REV. 7-73)

\begin{tabular}{|c|c|c|c|}
\hline $\begin{array}{l}\text { U.S. DEPT. OF COMM. } \\
\text { BIBLIOGRAPHIC DATA } \\
\text { SHEET }\end{array}$ & $\begin{array}{l}\text { 1. PURLICATION OR REPORT NO. } \\
\text { NBSIR } 75-823\end{array}$ & $\begin{array}{l}\text { 2. Gov't Accession } \\
\text { No. }\end{array}$ & 3. Recipient's Accession No. \\
\hline \multirow{2}{*}{\multicolumn{3}{|c|}{$\begin{array}{l}\text { 4. TITLE AND SUBTITLE } \\
\text { HELIUM RESEARCH IN SUPPORT OF SUPERCONDUCTING POWER } \\
\text { TRANSMISSION }\end{array}$}} & $\begin{array}{l}\text { 5. Publication Date } \\
\text { October } 1975\end{array}$ \\
\hline & & & $\begin{array}{l}\text { 6. Performing Organization Code } \\
275.05\end{array}$ \\
\hline \multicolumn{3}{|c|}{$\begin{array}{l}\text { 7. AUTHOR(S) V. D. ArP, D. E. Daney, N. V. Frederick, M. C. Jones, } \\
\text { P. R. Ludtke, W. R. Parrish and R. L. Powell } \\
\text { 9. PERFORMING ORGANIZATION NAME AND ADDRESS } \\
\text { NATIONAL BUREAU OF STANDARDS } \\
\text { DEPARTMENT OF COMMERCE } \\
\text { WASHINGTON, D.C. } 20234\end{array}$} & $\begin{array}{l}\text { 8. Performing Organ. Report No. } \\
\text { NBS IR } 75-823 \\
\begin{array}{l}\text { 10. Project/Task/Work Unit No. } \\
2750551\end{array} \\
\text { 11. Contract/Grant No. } \\
\text { ERDA, E }(49-18)-2128\end{array}$ \\
\hline \multicolumn{3}{|c|}{$\begin{array}{l}\text { 12. Sponsoring Organization Name and Complete Address (Street, City, State, ZIP) } \\
\text { Electrical Energy Systems Division } \\
\text { U.S. Energy Research and Development Administration } \\
\text { Washington, D.C. } 20545\end{array}$} & $\begin{array}{l}\text { 13. Type of Report \& Period } \\
\text { Covered Annual } \\
\text { Sept. 1974-July } 1975 \\
\text { 14. Sponsoring Agency Code }\end{array}$ \\
\hline
\end{tabular}

\section{SUPPLEMENTARY NOTES}

16. ABSTRACT (A 200-word or less factual summary of most significant information. If document includes a significant bibliography or literature survey, mention it here.)

This is the first annual report on a program of helium related research in support of superconducting power transmission. Program areas reported on are concerned with the dynamics of helium cooling systems --theoretical aspects of oscillatory behavior and experiments - - and helium related measurements. The latter include pressure measurement, temperature measurement and the problem of impurity levels in the helium refrigerant.

17. KEY WORDS (six to twelve entries; alphabetical order; capitalize only the first letter of the first key word unless a proper name; separated by semicolons) Cooling system; cryogenics; helium-cooled electrical leads; helium impurities; microwave cavities; negative differential flow resistance; pressure measurement; system dynamics; thermometers.

\begin{tabular}{|c|c|c|}
\hline 18. AVAILABILITY $\quad$ X Unlimited & $\begin{array}{l}\text { 19. SECURITY CLASS } \\
\text { (THIS REPORT) }\end{array}$ & 21. NO. OF PAGES \\
\hline [-For Official Distribution. Do Not Release to NTIS & UNCL ASSIFIED & 80 \\
\hline $\begin{array}{l}\text { Order From Sup. of Doc., U.S. Government Printing Office } \\
\text { Washington, D.C. } 20402, \text { SD Cat. No.C } 13\end{array}$ & $\begin{array}{l}\text { 20. SECURITY CLASS } \\
\text { (THIS PAGE) }\end{array}$ & 22. Price \\
\hline $\begin{array}{l}\text { X Order From Narional Technical Information Service (NTIS) } \\
\text { Springfield, Virginia } 22151\end{array}$ & UNCLASSIFIED & $\$ 4.75$ \\
\hline
\end{tabular}

